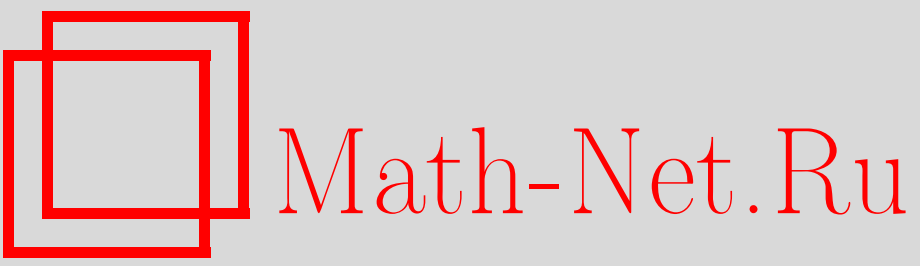

Д. Реповш, А. Б. Скопенков, Новые результаты о вложениях полиэдров и многообразий в евклидовы пространства, УМН, 1999, том 54, выпуск 6, 61-108

DOI: https://doi.org/10.4213/rm230

Использование Общероссийского математического портала Math-Net.Ru подразумевает, что вы прочитали и согласны с пользовательским соглашением

http://www.mathnet.ru/rus/agreement

Параметры загрузки:

IP: 52.87 .193 .239

26 апреля 2023 г., 11:58:24 


\title{
НОВЫЕ РЕЗУЛЬТАТЫ О ВЛОЖЕНИЯХ ПОЛИЭДРОВ И МНОГООБРАЗИЙ В ЕВКЛИДОВЫ ПРОСТРАНСТВА
}

\author{
Д. РеповШ, А. Скопенков
}

Цель обзора - изложить некоторые классические результаты о вложениях и изотопиях полиэдров и многообразий в $\mathbb{R}^{m}$, а также рассказать о современном возрождении интереса к этой красивой области топологии. Рассказывается о новых результатах в этой области: об усилении теоремы Хэфлигера-Вебера о полноте препятствия взрезанного квадрата для вложимости и изотопии высокосвязных многообразий в $\mathbb{R}^{m}$ (Скопенков), о невозможности ее усилить для полиэдров (Фридман, Крушкаль, Тайхнер, Сегал, Скопенков, Спеш) и многообразий, не имеющих достаточной связности (Скопенков). Показывается, как алгебраические препятствия (в терминах когомологий, характеристических классов и эквивариантных отображений) возникают при рассмотрении геометрических проблем о вложимости в евклидовы пространства. Формулируются и доказываются некоторые классические и современные результаты о полноте и неполноте этих препятствий. На примере этих доказательств иллюстрируются классические и современные методы геометрической топологии (поглощение, трюк Уитни, пальевые движения Ван Кампена и Кэссона и их обобщения).

Библиограффия: 167 названий.

\section{СОДЕРЖАНИЕ}

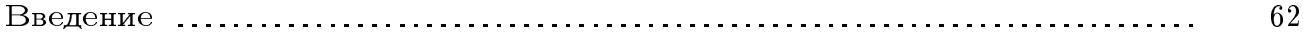

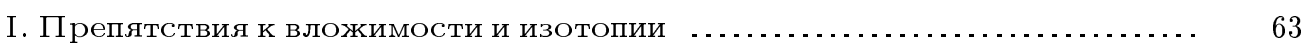

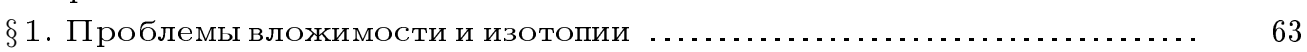

$\S 2$. Препятствие Ван Кампена ................................... 65

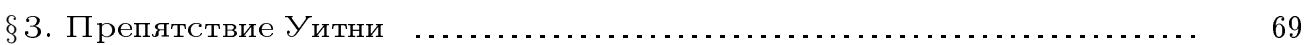

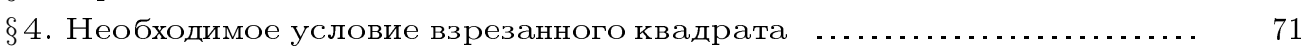

$\S 5$. Приложение: базисные вложения в плоскость $\ldots \ldots \ldots \ldots \ldots \ldots \ldots \ldots \ldots . . \ldots 77$

II. Исследование полноты препятствия взрезанного квадрата . . . . . . . . 80

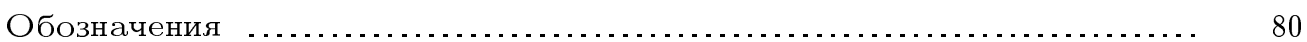

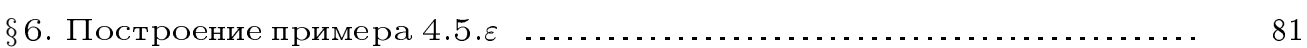

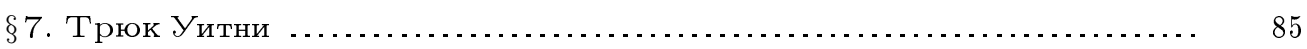

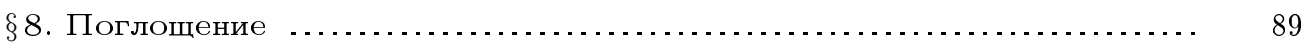

$\S 9$. Первая часть доказательства теоремы 4.1.е .................... 92

$\S 10$. Вторая часть доказательства теоремы 4.1.е ...................... 94

$\S 11$. Идея доказательства теоремы 4.2.е .......................... 98

$\S 12$. Идея доказательства изотопических версий $\ldots \ldots \ldots \ldots \ldots \ldots \ldots \ldots \ldots . . \ldots 1$

Список литературы ............................................ 102

(C) Д. Реповш, А. Скопенков 1999 


\section{Введение}

Многие теоремы в математике утверждают, что любое пространство из данного абстрактно определенного класса всегда является подпространством некоторого “стандартного” пространства из этого класса. Это такие теоремы, как теорема Кэли о вложении конечных групп в симметрическую группу, теорема о существовании точного линейного представления компактных групп Ли, теорема Урысона о вложении нормальных пространств со счетным базисом в гильбертово пространство, теорема обшего положения о вложении конечномерных полиэдров в $\mathbb{R}^{m}$, теорема Менгера-Нёбелинга-Понтрягина о вложении конечномерных компактов в $\mathbb{R}^{m}$, теорема Уитни о вложении гладких многообразий в $\mathbb{R}^{m}$, теорема Нэша о вложении римановых многообразий в $\mathbb{R}^{m}$, теорема Громова о вложении симплектических многообразий в $\mathbb{R}^{2 n}$ и т. д. Решение тринадшатой проблемы Гильберта А.Н. Колмогоровым и В.И. Арнольдом можно также сформулировать на языке вложений ( $(5)$. Эти теоремы вложимости интересны не только сами по себе, но и как сильные инструменты для решения других задач. Одними из главных классических проблем топологии являются более тонкие проблемы сушествования вложения и классификации вложений (с точностью до изотопии) данного пространства в $\mathbb{R}^{m}$ для данного $\mathrm{m}$.

В этом обзоре среди огромного количества методов и результатов по проблемам вложимости и изотопии выбран метод взрезанного квадрата (а также исторически предшествовавшие ему его "частные случаи" - методы Ван Кампена и Уитни). Метод взрезанного квадрата является проявлением общематематической идеи “дополнения до диагоналей” (Борсук, Ву, Лефшец, Шапиро). Классическая теорема Хэфлигера-Вебера (1963-1967 гг.) утверждает достаточность условия взрезанного квадрата для вложимости полиэдров и многообразий (и изотопности вложений полиэдров и многообразий) при "метастабильном" размерностном ограничении $m \geqslant \frac{3(n+1)}{2}$ $\left(m \geqslant \frac{3 n}{2}+2\right)$. В 1995-1998 гг. вторым автором было получено полное решение известной проблемы о возможности ослабить метастабильное ограничение в указанных теоремах для кусочно-линейных многообразий и полиэдров (более подробно см. § 2-4). Этот результат был получен за счет синтеза и усиления методов и результатов из разных разделов топологии: теории погружений, теории гомотопий, поглошения, обобшенного трюка Уитни, обобшенных пальцевых движений Ван Кампена и пальцевых движений Кэссона. Настоящий обзор посвящен изложению теоремы Хэфлигера-Вебера, указанных результатов второго автора и их следствий, а также связи проблемы вложения с другими областями математики (в частности, с алгебраической топологией и функциональньм анализом).

Первая часть начинается с постановки и обсуждения указанных проблем ( 1 1). О методах Ван Кампена и Уитни и соответствующих результатах рассказьвается в $\S 2,3$. В $\S 4$ формулируется классический результат Хэфлигера-Вебера о достаточности условия взрезанного квадрата для вложимости $n$-мерных пространств в $\mathbb{R}^{m}$ при $m \geqslant$ $\frac{3(n+1)}{2}$ (и для изотопности вложений при $m>\frac{3(n+1)}{2}$ ) без дополнительных предположений о высокой связности. Также формулируются результаты о достаточности этого условия при $m<\frac{3(n+1)}{2}$ и дополнительных предположениях высокой связнос-

Д. Реповш частично поддержан Министерством Науки и Технологии Республики Словения, гранты № J1-0885-0101-98 и SLO-US 0020. А. Скопенков частично поддержан Российским фондом фундаментальных исследований, грант № 99-01-00009. 
ти. В $\S 2-4$ приводится большое количество следствий этих результатов. Формулируется также теорема Хэфлигера-Хирша о погружениях многообразий в $\mathbb{R}^{m}$ и ее кусочно-линейный аналог, доказанньй вторым автором. В $\S 5$, не связанном с остальной частью обзора, обсуждаются современные результаты в теории базисных вложсний, возникшей на стыке функционального анализа и топологии в ходе исследования 13-й проблемы Гильберта.

Вторая часть посвящена доказательству теорем полноты (Хэфлигер, Вебер, Скопенков) и неполноты (Фридман, Крушкаль, Тайхнер, Сегал, Скопенков, Спеш) препятствия взрезанного квадрата. На примере доказательства этих результатов рассказывается о классических и современных методах геометрической топологии-трюке Уитни, поглощении, пальцевых движениях Ван Кампена (и их обобщениях). Эти методы применяются не только для изучения вложений. В $\S 6$ строятся примеры неполноты препятствий Ван Кампена и взрезанного квадрата при $4 \leqslant m \leqslant \frac{3 n}{2}+1$, анонсированные в $\S 2,4$. Построение этих примеров использует многомерные пальцевые движения Кэссона. Доказательства полноты препятствия взрезанного квадрата проводятся для кусочно-линейного случая (принадлежащие Веберу и второму автору). Для удобства читателя выбран исторический подход к изложению доказательств, при котором каждый метод сначала иллюстрируется на простейшем частном случае и только потом применяется в полной моши. В $\S 7$ на примере доказательств теоремы 2.1.а Ван Кампена-Шапиро-Ву для $n \geqslant 3$, а также теоремы 4.1.е Вебера для $m=2 n+1$ иллюстрируются трюк Уитни и пальцевые движения Ван Кампена. В $\S 8$ на примере доказательства теоремы 3.2.а Пенроуза-Уайтхеда-Зимана-Ирвина иллюстрируется метод поглощения. В $\S 9, \S 10$ методы $\S 7, \S 8$ применяются для доказательства общего случая $m \geqslant \frac{3(n+1)}{2}$ теоремы 4.1.е Вебера. Изложение второй части доказательства в $\S 10$ основано на методе из [136]. Оно существенно более простое по сравнению с оригинальным [153] и не использует теорему Фрейденталя о надстройке. В $\S 11$ приводится идея доказательства усиления теоремы 4.1.е Вебера для кусочно-линейного случая (теоремы 4.2.e) и кусочно-линейной версии теоремы 4.1.і Хэфлигера-Хирша (теоремы 4.2.i). В 12 приводится идея доказательства изотопических версий указанных результатов.

\section{I. Препятствия к вложимости и изотопии}

\section{§ 1. Проблемы вложимости и изотопии}

Компакт $K$ называется топологически (TOP), кусочно-линейно (PL) или гладко (DIFF) вложимым в $\mathbb{R}^{m}$, если существует непрерывное (TOP), кусочно-линейное $(\mathrm{PL})$ или гладкое $(\mathrm{DIFF})$ инъективное отображение $f: K \rightarrow \mathbb{R}^{m}$ (в гладком случае дополнительно требуется, чтобы $d f$ был мономорфизмом в каждой точке). Такое отображение $f$ назьвается вложсением $K$ в $\mathbb{R}^{m}$. Первая главная проблема, которой посвяшен этот обзор, - найти условия для того, чтобы $K$ был вложим в $\mathbb{R}^{m}$ для данного $m$. Рассматривается в основном (кроме §5) PL случай, в частности, все отображения предполагаются PL, если не указано противное. Напомним, что отображение $f: K \rightarrow \mathbb{R}^{m}$ полиэдра $K$ назьвается кусочно-линейным, если оно линейно на симплексах некоторой триангулящии полиэдра $K$. Результаты и ссылки в $\S 3$ и $\S 4$, относящиеся к DIFF случаю, приведены в круглых скобках. Под полиэдром всегда под- 
разумевается конечный полиэдр. Прекрасньй обзор DIFF вложимости - [35]. Когда $m \geqslant 2 \operatorname{dim} K+1$, полиэдр $K$ вложим в $\mathbb{R}^{m}$ по соображениям общего положения (на самом деле, даже $K \times I$ вложим в $\left.\mathbb{R}^{m}[122]\right)$. Итак, указанная проблема интересна только для $m \leqslant 2 \operatorname{dim} K$.

Близкие понятия, которые интересны сами по себе и полезны для изучения вложений, - погружения и квазивложения. Их определения получаются ослаблением условия инъективности в определении вложения двумя двойственными способами. Полиэдр $K$ называется погружаемым (соответственно квазивложимылм) в $\mathbb{R}^{m}$, если для любого $\varepsilon>0$ сушествует отображение $f: K \rightarrow \mathbb{R}^{m}$ такое, что $f(x) \neq f(y)$ при $\operatorname{dist}(x, y)<\varepsilon($ соответственно при $\operatorname{dist}(x, y)>\varepsilon)$. Для $m-n \geqslant 3$ любое вложение $\mathrm{PL}$ $n$-многообразия в $\mathbb{R}^{m}$ локально плоско, значит, наше определение совпадает с принятым для PL многообразий [54]. Многие результаты для вложений имеют аналоги для погружений и квазивложений.

Другая интересная проблема состоит в определении, когда два заданных вложения $f, g: K \rightarrow \mathbb{R}^{m}$ "одинаковы". Наиболее известна задача о классификации узлов в $\mathbb{R}^{3}$. Более точно, два TOP, PL или DIFF вложения $f, g: K \rightarrow \mathbb{R}^{m}$ назьваются (облемлемо) изотопнылми, если сушествует TOP, PL или DIFF гомеоморфизм (на) $F: \mathbb{R}^{m} \times I$ $\rightarrow \mathbb{R}^{m} \times I$ такой, что $F(y, 0)=(y, 0)$ для любого $y \in \mathbb{R}^{m}, F(f(x), 1)=(g(x), 1)$ для любого $x \in K$ и $F\left(\mathbb{R}^{m} \times\{t\}\right)=\mathbb{R}^{m} \times\{t\}$ для любого $t \in I$. Этот гомеоморфизм $F$ назьвается (объемлющей) изотопией. (Объемлющей) изотопией также назьвают гомотопию $\mathbb{R}^{m} \times I \rightarrow \mathbb{R}^{m}$ или семейство отображений $F_{t}: \mathbb{R}^{m} \rightarrow \mathbb{R}^{m}$, очевидным образом порожденные отображением $F$. Вторая классическая проблема топологии, которой посвящен этот обзор, - найти условие того, что вложения $f, g: K \rightarrow \mathbb{R}^{m}$ изотопны (как и для проблемывложения, в основном будет рассматриваться PL случай). Когда $m \geqslant 2 \operatorname{dim} K+2$, любые два вложения полиэдра $K$ в $\mathbb{R}^{m}$ изотопны по соображениям общего положения. Аналогично, два TOP, PL или DIFF погружения $f, g: K \rightarrow \mathbb{R}^{m}$ называются регулярно гомотопными, если существует TOP, PL или DIFF погружение $F: K \times I \rightarrow \mathbb{R}^{m} \times I$ такое, что $F(x, 0)=(f(x), 0), F(x, 1)=(g(x), 1)$ для любого $x \in K$ и $F(K \times\{t\}) \subset \mathbb{R}^{m} \times\{t\}$ для любого $t \in I$.

Очевидно, (объемлющая) изотопия - это отношение эквивалентности на множестве вложений $K$ в $\mathbb{R}^{m}$. Это сильнейшее отношение эквивалентности среди (не объемлюшей) изотопии, изопозиции, конкордантности, бордантности и т. д. Два вложения $f, g: K \rightarrow \mathbb{R}^{m}$ назьваются (не объемлемо) изотопнылми, если существует вложение $F: K \times I \rightarrow \mathbb{R}^{m} \times I$ такое, что $F(x, 0)=(f(x), 0), F(x, 1)=(g(x), 0)$ для любого $x \in K$ и $F(K \times\{t\}) \subset \mathbb{R}^{m} \times\{t\}$ для любого $t \in I$. В DIFF категории или при $m-\operatorname{dim} K \geqslant 3$ в TOP и PL категориях из изотопности следует объемлемая изотопность [4], [26; §7], [66], [74]. При $m-\operatorname{dim} K \leqslant 2$ это не так (например, любой узел $S^{1} \rightarrow S^{3} \mathrm{PL}$ изотопен тривиальному, но не обязательно объемлемо изотопен ему). Два вложения $f, g: K \rightarrow \mathbb{R}^{m}$ назьваются (ориентированно) изопозиционньмu, если существует (сохраняющий ориентацию) гомеоморфизм $F: \mathbb{R}^{m} \rightarrow \mathbb{R}^{m}$ такой, что $F \circ f=g$. Теорема Александера-Гугенхайма утверждает, что ориентированная изопозиция эквивалентна изотопности [125]. Два вложения $f, g: K \rightarrow \mathbb{R}^{m}$ назьваются объемлемо конкордантнылми, если существует гомеоморфизм (на) $F: \mathbb{R}^{m} \times I$ $\rightarrow \mathbb{R}^{m} \times I$ (который называется конкордантностью) такой, что $F(y, 0)=(y, 0)$ для любого $y \in \mathbb{R}^{m}$ и $F(f(x), 1)=(g(x), 1)$ для любого $x \in K$. Определение не обгемлемой конкордантности получается из определения не объемлемой изотопии ана- 
логично, отбрасьванием последнего условия сохранения уровней. B DIFF категории или при $m-\operatorname{dim} K \geqslant 3$ в TOP и PL категориях из конкордантности следует объемлемая конкордантность [70], [73], [165] (это неверно в случае коразмерности один или два). Этот результат позволяет редуцировать проблему изотопии к относительной проблеме вложимости (подробнее см. 112 ).

Приведем (заведомо неполные) ссылки на литературу по близким вопросам геометрической топологии. В проблемах вложимости и изотопности пространство $\mathbb{R}^{m}$ может быть заменено на произвольное пространство $Y$. Случаи, когда $Y$ - многообразие или произведение деревьев, были наиболее широко рассмотрены [145; теорема 4.6 и комментарии], [33], [34], [167]. О вложении прямых и непрямых произведений и әрупп Ли см. [2], [116], [117], [121]. О вложениях с точностью до кобордизма см. [11]. О вложениях с точностью до гомотопии см. [152; $\S 11]$. О классификации сингулярных зацеплений см. [40], [138] и библиографию в этих работах. О задаче вложимости полиэдров в некоторые многообразия см. [10], [97], [108], [133], [150], [151] и библиографию там. О задачах вложимости компактов и близкой задаче аппроксимации вложсниями см. [3], [16], [17], [23], [104], [105], [118; §9], [119], [129] и библиографию там. О задаче пересечения компактов см. [25], [140] и библиографию там. О базисныл вложениях см. $\S 5$.

\section{§. Препятствие Ван Кампена}

Как показано в $§ 1$, первым нетривиальным случаем проблемы вложимости является исследование вложимости $n$-полиэдров в евклидово пространство $\mathbb{R}^{2 n}$. Для $n=1$ эта проблема решается критерием Понтрягина-Куратовского [89] (см. также [118; § 2] и библиографию там). Однако для $n>1$ такого простого критерия не существует (так как, например, имеется бесконечное количество замкнутых неориентируемых 2-поверхностей, которые не вложимы в $\mathbb{R}^{3}$ и ни одна из которых не содержит другую; см. также [128]. В работе [80] построено препятствие к вложимости $n$-полиэдров в $\mathbb{R}^{2 n}$ при произвольном $n$ и предприняты шаги к доказательству его полноты (см. теорему 2.1.а ниже и историческое замечание в конце этого параграфа).

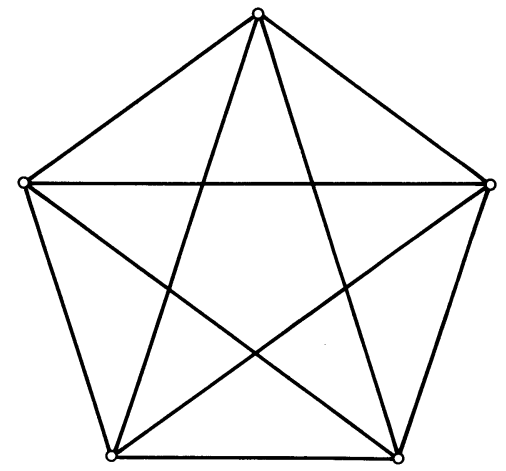

Рис. 2.1 
Чтобы объяснить идею построения препятствия Ван Кампена, приведем набросок доказательства непланарности графа $K_{5}$, т.е. полного графа с 5 вершинами (рис. 2.1). Возьмем любое отображение общего положения $f: K_{5} \rightarrow \mathbb{R}^{2}$. Для любых двух ребер $\sigma, \tau$ пересечение $f \sigma \cap f \tau$ состоит из конечного числа точек. Пусть $v_{f}$ будет суммой по модулю 2 чисел $|f \sigma \cap f \tau|$ по всем неупорядоченным парам $\{\sigma, \tau\}$ несмежных ребер графа $K_{5}$. Для отображения $f$, показанного на рис. 2.1, $v_{f}=1$. Любое отображение обшего положения $f: K_{5} \rightarrow \mathbb{R}^{2}$ может быть преобразовано к любому другому такому через изотопии плоскости $\mathbb{R}^{2}$ и “движения Райдемайстера" для графов на плоскости, изображенные на рис. 2.2. Это утверждение доказьвается аналогично теореме Райдемайстера для узлов. Мы не будем его доказьвать, так как оно нужно нам только для пояснения идеи и не используется в строгих доказательствах. Для каждого ребра граффа $K_{5}$ с вершинами $a, b$ граф $K_{5}-\{a, b\}$, полученньй удалением из $K_{5}$ вершин $a, b$ и внутренностей ребер, выходяших из $a, b$, есть окружность (это то самое свойство графиа $K_{5}$, которое необходимо для доказательства). Следовательно, $v_{f}$ - инвариант “движений Райдемайстера". Значит, $v_{f}=1$ для кажсдого отображения $f: K_{5} \rightarrow \mathbb{R}^{2}$. Следовательно, $K_{5}$ непланарен.
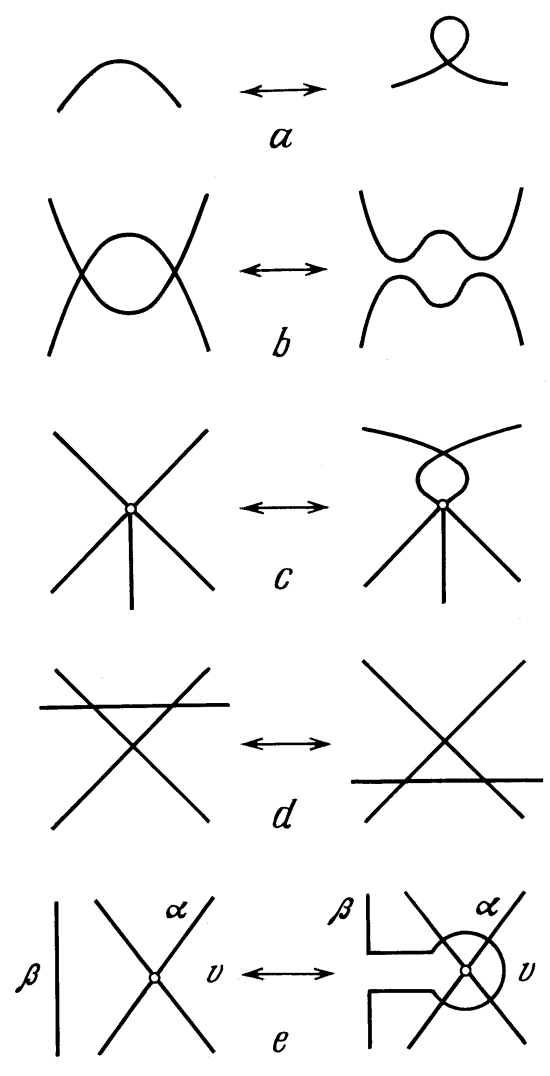

Рис. 2.2

Теперь обсудим некоторые обобшения этого доказательства, которые будут использованы впоследствии. Из этого доказательства на самом деле получается более 


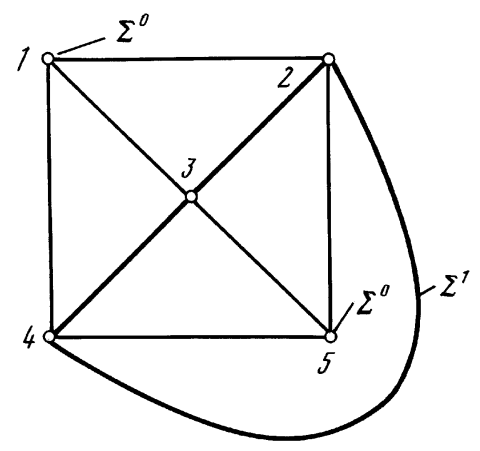

Рис. 2.3

сильное утверждение. Пусть $e$ - ребро графа $K_{5}$ и $\Sigma$ - цикл в графе $K_{5}$, образованный ребрами графа $K_{5}$, не смежными с $e$. Обозначим $\stackrel{\circ}{e}=\operatorname{Int} e$. Тогда граф $K_{5}-\stackrel{\circ}{e}$ вложим в $\mathbb{R}^{2}$ (рис. 2.3 ) и для любого вложения $g: K_{5}-\stackrel{\circ}{e} \rightarrow \mathbb{R}^{2} g$-образы концов ребра $e$ (0-сферы) лежат по разные стороны от $g \Sigma$. Подобным образом можно доказать, что граф $K_{33}$ (три домика и три колодша) не вложим в $\mathbb{R}^{2}$ и что 2 -остов $K 6$-симплекса не вложим в $\mathbb{R}^{4}$. Более того, пусть $e-$ некоторый 2 -симплекс этого полиэдра $K$ и $P=K-\stackrel{\circ}{e}$. Тогда $P$ вкладывается в $\mathbb{R}^{4}$ и содержит две такие непересекаюшиеся сферы $\Sigma^{2}$ и $\Sigma^{1}=\partial e$, что для любого вложения $P \rightarrow \mathbb{R}^{4}$ образы этих сфер зацеплены с ненулевьм (конкретнее, равным \pm 1 ) коэффищиентом зацепления [28].

Введем основное определение этого параграфа. Пространство

$$
\widetilde{T}=\bigcup\{\sigma \times \tau \in T \times T \mid \sigma \cap \tau=\varnothing\}
$$

назьвается симплициальным взрезаннылм квадратом полиэдра $K$. Так как эквивариантньй гомотопический тип пространства $\widetilde{T}$ зависит только от $K$ [132; лемма 2.1$]$, $[63 ; \S 4]$, то в этом параграфе мы заменяем $\widetilde{T}$ на $\widetilde{K}$. Группа $\mathbb{Z}_{2}$ действует на $\widetilde{K}$ перестановкой множителей. Пусть $K^{*}=\widetilde{K} / \mathbb{Z}_{2}$.

Теперь мы в состоянии описать препятствие Ван Кампена $v(K)$ для $n=1$. Повсюду в этом параграфе мы будем опускать $\mathbb{Z}_{2}$-коэффишиенты из обозначений групп (ко)цепей и (ко)гомологий. Зафиксируем триангулящию $T$ граффа $K$. Для любого отображения $f: K \rightarrow \mathbb{R}^{2}$ общего положения и любых двух несмежных ребер $\sigma, \tau$ из $K$ пересечение $f \sigma \cap f \tau$ состоит из конечного числа точек. Пусть $v_{f}(\sigma, \tau)=|f \sigma \cap f \tau| \bmod 2$. Тогда $v_{f} \in C^{2}\left(K^{*}\right)$. Эта коцепь $v_{f}$ является инвариантом относительно изотопий плоскости $\mathbb{R}^{2}$ и первых четырех “движений Райдемайстера" (рис. 2.2.a-d). При пятом “движении Райдемайстера" (рис. 2.2.e) к $v_{f}$ добавляется коцепь, которая принимает значение 1 на классе 2-симплекса $\alpha \times \beta$ для $v \in \alpha$ и равна нулю на остальных 2 -симплексах. Эта коцепш есть кограница $\delta[v \times \beta]$ элементарной когранищы из $B^{2}\left(K^{*}\right)$, которая принимает значение 1 на классе 1 -симплекса $v \times \beta$ и 0 на остальных 1-симплексах. Тогда класс

$$
v_{2}(K)=\left[v_{f}\right] \in H^{2}\left(K^{*}\right)=C^{2}\left(K^{*}\right) / B^{2}\left(K^{*}\right)
$$


не зависит от $f$ (поскольку $\operatorname{dim} K^{*}=2$, то $\left.C^{2}\left(K^{*}\right)=Z^{2}\left(K^{*}\right)\right)$. Полученный класс когомологий $v_{2}(K)$ и есть препятствие Ван Кампена для вложимости $K$ в $\mathbb{R}^{2}$ (точнее, его аналог $\bmod 2)$. Понятно, что $v_{2}(K)=0$ для любого планарного графа $K$.

Приведем доказательство независимости $\left[v_{f}\right]$ от $f$ без использования не доказанного здесь факта о “движениях Райдемайстера", следуя [132; лемма 3.5]. Для заданных отображений $f_{0}, f_{1}: K \rightarrow \mathbb{R}^{2}$ обшего положения рассмотрим произвольную линейную гомотопию $f: K \times I \rightarrow \mathbb{R}^{2}$ общего положения. Определим коцепь $v_{f} \in C^{2 n-1}\left(K^{*}\right)$ формулой $v_{f}\left[\sigma^{n} \times \tau^{n-1}\right]=\left|f_{0}(\sigma) \cap f(\tau \times I)\right| \bmod 2$. Тогда независимость $v_{f}$ от $f$ следует из легко проверяемого равенства $v_{f_{0}}-v_{f_{1}}=\delta v_{f}$.

Аналогично определяется $\bmod 2$ препятствие Ван Кампена $v_{2}(K) \in H^{2 n}\left(K^{*}\right)$ к вложимости $n$-полиэдра $K$ в $\mathbb{R}^{2 n}$. Настоящее препятствие Ван Кампена $v(K)$ (с целыми коэффициентами) строится так. Зафиксируем триангулящию полиэдра $K$ и определим $\widetilde{K}$ и $K^{*}$ как и выше. Выберем ориентацию в $\mathbb{R}^{2 n}$ и на $n$-симплексах $K$. Для любого отображения $f: K \rightarrow \mathbb{R}^{2 n}$ общего положения и любых двух несмежных ориентированных $n$-симплексов $\sigma, \tau$ из $K$ пересечение $f \sigma \cap f \tau$ состоит из конечного числа точек. Определим коцеп $v_{f} \in C^{2 n}(\widetilde{K}, \mathbb{Z})\left(\right.$ индекс пересечения) формулой $v_{f}(\sigma, \tau)=$ $f \sigma \cdot f \tau=\sum_{P \in f \sigma \cap f \tau} \operatorname{sign} P$, где $\operatorname{sign} P=+1$, если $n$-реперы ориентаций $f \sigma$ и $f \tau$ (в этом порядке) составляют $2 n$-репер ориентации $\mathbb{R}^{2 n}$, и $\operatorname{sign} P=-1$ в противном случае. Очевидно, $v_{f}(\sigma, \tau)=(-1)^{n} v_{f}(\tau, \sigma)$. Поэтому $v_{f}$ находится в подгруппе $C_{s}^{2 n}(\widetilde{K}, \mathbb{Z})<C^{2 n}(\widetilde{K}, \mathbb{Z})$ коцепей, принимающих на $2 n$-клетках $\sigma \times \tau$ и $\tau \times \sigma$ равные значения для четных $n$ и противоположные значения для нечетных $n$. Класс когомологий

$$
v(K)=\left[v_{f}\right] \in H_{s}^{2 n}(\widetilde{K}, \mathbb{Z})=C_{s}^{2 n}(\widetilde{K}, \mathbb{Z}) / B_{s}^{2 n}(\widetilde{K}, \mathbb{Z})
$$

(аналогично $v_{2}(K)$, не зависящий от $f$ ) назьвается препятствием Ван Кампена для вложимости полиэдра $K$ в $\mathbb{R}^{2 n}$. Заметим, что $H_{s}^{2 n}(\widetilde{K}, \mathbb{Z}) \cong H^{2 n}\left(K^{*}, \mathbb{Z}\right)$ для четного $n[29]$. Нетрудно показать, что $v(K)$ зависит от выбора ориентаций в $\mathbb{R}^{2 n}$ и на $n$-симплексах полиэдра $K$ лишь с точностью до автоморфизма группы $H_{s}^{2 n}(\widetilde{K}, \mathbb{Z})$.

Предыдушие построения могут быть обобшены различными путями. Для подполиэдра $A$ полиэдра $K$ мы можем аналогично определить препятствие к продолжению вложения $A \rightarrow \partial B^{m}$ до вложения $K \rightarrow B^{m}$ [29]. Аналогично можно построить различающий класс $u(f) \in H_{s}^{2 n}(\widetilde{K}, \mathbb{Z})$ вложения $f: K \rightarrow \mathbb{R}^{2 n+1}[147]$. О препятствии Ван Кампена для аппроксимации вложсениями см. [15; § 4], [119], [3], [121].

Tеорема 2.1. а) [80], [132], [159], [127], [29]. Для вложимости конечного $n$-полиәдра $K$ в $\mathbb{R}^{2 n}$ необходимо, чтобь $v(K)=0$. При $n \neq 2$ это условие достаточно, а при $n=2$ - нет.

b) [160] Если вложения $f, g: K \rightarrow \mathbb{R}^{2 n+1}$ конечного $n$-полиэдра $K$ изотопньи, то $u(f)=u(g)$. При $n \geqslant 2$ это условие достаточно, а при $n=1$ - нет (в то же время, при $n=1$ вложсения $f, g$ с $u(f)=u(g)$ гомологичны [147].

Относительное препятствие Ван Кампена полно для $n \neq 2$ (для $n \geqslant 3$ см. [160], для $n=1$ это следует из относительной версии теоремы Понтрягина-Куратовского) и неполно для $n=2$ (см. $\S 6$ ). Из теоремы 2.1.а следует формулируемая ниже теорема 3.1 в PL категории (хронологически доказанная ранее теоремы 2.1.a). Для $n \geqslant 3$ интересным следствием теоремы 2.1.а является то, что любой ацикличный $n$-полиэдр $\mathrm{PL}$ вложим в $\mathbb{R}^{2 n}[159]$, см. также [60]. Для $n=2$ независимо от теоремы 2.1.а доказывается, что любой ацикличный 2-полиэдр ТОР вложим в $\mathbb{R}^{4}$ [85]. Теорема 2.1.а 
может быть применена для вычисления минимального $m$, для которого полиэдр, являющийся произведением графов, вложим в $\mathbb{R}^{m}[2],[32]$.

В этой работе мы не приводим доказательства теоремы 2.1.b (теорема 2.1.b для $n \geqslant 3$ доказьвается аналогично теореме 2.1.а с использованием идей из $\S 12$, а для $n=1$ см. [147]. Необходимость в теореме 2.1.а была доказана при построении препятствия Ван Кампена. Достаточность в теореме 2.1.а для $n \geqslant 3$ доказывается в $\S 7$, а для $n=1$ является следствием критерия Понтрягина-Куратовского планарности графов. Неполнота препятствия Ван Кампена для $n=2$ доказывается в $\S 6$.

\section{§. Препятствие Уитни}

TeOpema 3.1 [80], [158]. Любое PL (DIFF) n-многообразие PL (DIFF) вложимо в $\mathbb{R}^{2 n}$.

Доказательство теоремы 3.1 для PL (DIFF) случая приводится в $\S 8$ (§ 7). Размерность $2 n$ в теореме 3.1 является наилучшей для $n=2^{k}$ (так как $\mathbb{R} P^{n}$ не вложимо в $\mathbb{R}^{2 n-1}$ ) и не является наилучшей для других $n$ (см. следствие 3.4 ниже). Знаменитая и трудная гипотеза состоит в том, что любое замкнутое $n$-многообразие вложимо в $\mathbb{R}^{2 n-\alpha(n)+1}$, где $n=2^{k_{1}}+\cdots+2^{k_{\alpha(n)}}$ и $k_{1}<\cdots<k_{\alpha(n)}$ (о решении аналогичной гипотезы для погружений см. [21], [93]). Заметим, что $n$-многообразие $\mathbb{R} P^{2^{k_{1}}} \times \cdots \times \mathbb{R} P^{2^{k} \alpha(n)}$ не вложимо в $\mathbb{R}^{2 n-\alpha(n)}$.

Teорема 3.2. а) [42], [112], [78] Любое замкнутое $(2 n-m)$-связное PL (DIFF) $n$-многообразие $N$ PL (DIFF) вложимо в $\mathbb{R}^{m}$, если $m \geqslant n+3\left(m \geqslant \frac{3(n+1)}{2}\right)$.

b) [42], [78] Предположим, что $N$ и $M-(2 n-m)-u(2 n-m+1)$-связнье $P L(D I F F)$ п-многообразия соответственно (имеющие край или замкнутые) $u f: N \rightarrow M$ - такое отображение, что $\left.f\right|_{\partial N}$ является вложсением в Тогда $f$ PL (DIFF) гомотопно вложению $\operatorname{rel} \partial N$ nри $m \geqslant n+3\left(m \geqslant \frac{3(n+1)}{2}\right)$.

Доказательство теоремы 3.2 для PL случая приводится в $\S$ 8. Заметим, что PL случай теоремы 3.2.а неинтересен для $m<\frac{3 n}{2}+1$. В самом деле, тогда $2 n-m>\frac{n}{2}-1$ и $(2 n-m)$-связное многообразие $N$ является гомотопической сферой. Поскольку $\frac{3 n}{2}+1>m \geqslant n+3$, размерность $n \geqslant 6$. Значит, $N \cong S^{n}$, и теорема 3.2.а верна по очевидным соображениям. Заметим, что DIFF случай теоремы 3.2.а неверен для $m<\frac{3 n}{2}+1$ [61]. Обобщения теоремы 3.2 см. в [57], [65], [67], [41], [72], [38], [81], [13], [71], [95], [163] или ниже в этом параграфе.

Введем обозначения, используемые в $\S 3$ и $\S 4$. Для группы $G$ пусть $G_{(l)}$ (соответственно $\left.G_{[l]}\right)$ есть сама группа $G$ при четном $l$ и подгруппа элементов порядка 2 группы $G$ (соответственно $G / 2 G$ ) при нечетном $l$. Гомоморфизму $\varphi: G \rightarrow H$ соответствуют очевидные гомоморфизмы $\varphi_{(l)}: G_{(l)} \rightarrow H_{(l)}$ и $\varphi_{[l]}: G_{[l]} \rightarrow H_{[l]}$. Если $G-$ конечная абелева группа, то нетрудно видеть, что $G_{(l)} \cong G_{[l]}$.

Напомним некоторые определения. Замкнутое многообразие $N$ и пара $(N, \partial N)$ называются гомологически $k$-связными, если $N$ связно и, кроме того, $H_{i}(N)=0$ для любого $i=1, \ldots, k$ и $H_{i}(N, \partial N)=0$ для любого $i=0, \ldots, k$ соответственно. Для обоих случаев это условие равносильно тому, что $\widetilde{H}_{i}(X)=0$ при любом $i=0, \ldots, k$, где $X$ есть $N$ или $(N, \partial N)$ и $\widetilde{H}_{i}$ - приведенные гомологические групшы. Заметим, что если $H_{0}(N, \partial N)=0$, то многообразие $N$ не имеет замкнутых связных компонент. Мы 
используем следуюшие соглашения: 0-связность равносильна гомологической 0-связности и равносильна связности, $k$-связность для $k \leqslant 0$ есть 0 -связность.

В 1934 г. Уитни доказал, что для ориентируемого многообразия $N$ и погружения $f: N \rightarrow \mathbb{R}^{m}$ общего положения гомологический класс

$$
\bar{W}_{m-n}(N) \in H_{2 n-m}\left(N, \mathbb{Z}_{(m-n)}\right) \cong H^{m-n}\left(N, \mathbb{Z}_{(m-n)}\right)
$$

проекции сингулярного подмногообразия $\widetilde{\Delta}(f)=\{(x, y) \in N \times N \mid x \neq y, f x=f y\}$ на $N$ не зависит от $f$. Этот класс является препятствием Уитни к вложимости $N$ в $\mathbb{R}^{m}$ : если DIFF $n$-многообразие $N$ вложимо в $\mathbb{R}^{m}$, то $\bar{W}^{k}(N)=0$ для $k \geqslant m-n$ [156], см. также [107], [114], [124], [157].

Tеорема 3.3 ([53], [153], случай $m<\frac{3(n+1)}{2}-[135]$, см. также [69; §11]). Пусть $N$ - замкнутое $(2 n-m-1)$-связное $P L(D I F F)$ многообразие. При $m \geqslant n+3$ $\left(m \geqslant \frac{3(n+1)}{2}\right.$ или $\left.(m, n)=(12 l-1,8 l-1)\right)$ многообразие $N$ PL $(D I F F)$ вложимо в $\mathbb{R}^{m}$ тогда и только тогда, когда $\bar{W}_{m-n}(N)=0$.

Для случая $m \geqslant \frac{3 n+1}{2}$ условие гомотопической $(2 n-m-1)$-связности в теореме 3.3 можно заменить на условие гомологической $(2 n-m-1)$-связности. Теорема 3.3 следует из теорем 4.1.е и 4.2.е и следствия 4.3. Как и в теореме 3.2 , PL случай теоремы 3.3 неинтересен для $m<\frac{3 n}{2}$, поскольку из [83] следует, что любая $n$-мерная PL гомологическая сфера вложима в $\mathbb{R}^{n+1}$ (если $n \neq 3$ - то кусочно-линейно или гладко, если $n=3$ - то только топологически). Из теоремы 3.3 и [100], [101] это следует для $n \neq 3,4$ (для $n=3,4$ это было доказано отдельно):

СлЕДСТвИЕ 3.4. 1) [109], [53], [58], [150], [153], [8], [24], [27], [123] Любое замкнутое, ориентируемое для $n=2^{k} \quad P L(D I F F)$-многообразие $P L$ (DIFF) вложимо в $\mathbb{R}^{2 n-1}$.

2) ([46], [153], для $n=6-[135])$ Eсли $n \geqslant 6(n \geqslant 8)$ - четное число и $n \neq$ $2^{k}\left(2^{h}+1\right)$ для любъх иельх $k, h \geqslant 2$, то любое $P L(D I F F) n$-многообразие $N c$ $H_{1}(N)=0 P L(D I F F)$ влоэсимо в $\mathbb{R}^{2 n-2}$

3) [135], [137] Любое замкнутое гомологически 2-связное гладкое 7-многообразие гладко вложимо в $\mathbb{R}^{11}$.

Закончим формулировкой изотопических аналогов изложенных результатов.

Tеорема 3.5 [163], [42], [142], [36]. Любое PL (DIFF) [TOP, локально плоское] вложение $S^{n} \rightarrow S^{m}$ является $P L(D I F F)[T O P]$ незаузленнылм при $m-n \geqslant 3$ $\left(m \geqslant \frac{3(n+1)}{2}\right)[m \geqslant n+3]$.

В теореме 3.5 в квадратных скобках приведена формулировка для ТОР случая. В этой теореме размерностные ограничения точны, а предположение локальной плоскости в ТОР случае важно [43], [47], [126].

Tеорема 3.6. Пусть $N$ - замкнутое связное $P L$ (DIFF) n-многообразие и $m-n \geqslant 3\left(m \geqslant \frac{3 n}{2}+2\right)$.

1) [42], [166] Если $N$ является $(2 n-m+1)$-связньм. то любие два $P L(D I F F)$ вложения $N \rightarrow \mathbb{R}^{m} P L(D I F F)$ изотопнье. 
2) ([53], [153], [69], [7], [8], для $m \leqslant \frac{3(n+1)}{2}-$ [135]) Если $N$ ориентируемо и $(2 n-m)$-связно, то $P L(D I F F)$ изотопические классы вложский $N \rightarrow \mathbb{R}^{m}$ находятся во взаимно однозначном соответствии с $H^{m-n-1}\left(M, \mathbb{Z}_{(m-n)}\right)$.

3) ([53], [153], для $n=3-[135])$ Eсли $n \neq 2$ и $N$ неориентируемо, то $P L$ (DIFF) изотопические классы вложений $N \rightarrow \mathbb{R}^{2 n}$ находятся во взаимно однозначном соответствии с $H^{n-1}\left(M, \mathbb{Z}_{(n)}\right) \otimes \mathbb{Z}_{(n-1)}$.

Для случая $m \geqslant \frac{3 n}{2}+1$ условие гомотопической $(2 n-m)$-связности в теореме 3.6 можно заменить на условие гомологической $(2 n-m)$-связности.

\section{§4. Необходимое условие взрезанного квадрата}

Идея "дополнения до диагонали" играет важную роль в разных областях математики [31], [37], [149]. Необходимое условие взрезанного квадрата для вложимости и изотопии является реализацией этой идеи в теории вложений. Фактически оно восходит к двум знаменитым теоремам: теореме С. Лефшеца о неподвижной точке и теореме К. Борсука об антиподах [9].

Прежде чем сформулировать упомянутое необходимое условие, рассмотрим следующий пример. Докажем, что сфера $S^{n}$ не вложима в $\mathbb{R}^{n}$. Хотя этот факт очевиден, но приведенный здесь метод доказательства допускает широкое обобшение. Предположим противное, т.е. что сушествует вложение $f: S^{n} \rightarrow \mathbb{R}^{n}$. Определим отображение $\tilde{f}: S^{n} \rightarrow S^{n-1}$ формулой $\tilde{f}(x)=\frac{f(x)-f(-x)}{\|f(x)-f(-x)\|}$. Здесь $-x$ означает точку, противоположную к $x \in S^{n}$. Так как $f$-вложение, то $\tilde{f}$ определено корректно. Очевидно, что $\widetilde{f}$ эквивариантно по отношению к антиподальным инволюциям на $S^{n}$ и $S^{n-1}$. Ограничение $\left.\widetilde{f}\right|_{S^{n-1}}$ продолжается на $S^{n}$ и поэтому гомотопно нулю. Но по теореме Борсука-Улама любое эквивариантное отображение $S^{n-1} \rightarrow S^{n-1}$ не гомотопно нулю. Полученное противоречие доказьвает невложимость $S^{n}$ в $\mathbb{R}^{n}$. На самом деле, мы доказали даже, что сушествует такая пара точек $x$ и $-x$, что $f(x)=f(-x)$.

Чтобы сформулировать необходимое условие взрезанного квадрата, нам понадобится следующее определение. Взрезанным квадратом $\widetilde{N}$ топологического пространства $N$ назьвается его произведение на себя без диагонали:

$$
\widetilde{N}=\{(x, y) \in N \times N \mid x \neq y\}
$$

Пусть теперь $f: N \rightarrow \mathbb{R}^{m}$ - вложение полиэдра $N$ в евклидово пространство $\mathbb{R}^{m}$. Тогда корректно определено отображение $\widetilde{f}: \widetilde{N} \rightarrow S^{m-1}$, задаваемое формулой $\widetilde{f}(x, y)=$ $\frac{f(x)-f(y)}{\|f(x)-f(y)\|}$. Это отображение эквивариантно по отношению к инволюции $t(x, y)=$ $(y, x)$ на $N$ и антиподальной инволюции на $S^{m-1}$. Итак, необходимым условием взрезанного квадрата для вложимости $K$ в $\mathbb{R}^{m}$ назьвается сушествование хотя бы одного эквивариантного отображения $\widetilde{N} \rightarrow S^{m-1}$. Сушествование эквивариантного отображения $\widetilde{N} \rightarrow S^{m-1}$ эквивалентно сушествованию сечения расслоения $\widetilde{N} \times S^{m-1} /(t \times a) \underset{g}{\stackrel{S^{m-1}}{\longrightarrow}} \tilde{N} / t$, где $t$ - инволюция $(x, y) \leftrightarrow(y, x)$ на $\tilde{N}, a$ - антиподальная инволюция на сфере $S^{m-1}$ и отображение $g$ задано формулой $g[(x, y), \alpha]=[(x, y)]$. 
Если $N$ - полиэдр или гладкое многообразие, то условие Ву можно проверять, используя методы теории препятствий [22], [160], [44]. В частности, описанные вьше препятствия Ван Кампена и Уитни являются первыми препятствиями к построению сечения расслоения $g$, т.е. могут быть получены из условия взрезанного квадрата чисто алгебраическим путем. Так как симплициальный взрезанный квадрат $\widetilde{T}(\S 2)$ является эквивариантным ретрактом пространства $\widetilde{N}$ [63], то условие взрезанного квадрата эквивалентно существованию эквивариантного отображения $\widetilde{T} \rightarrow S^{m-1}$.

Необходимое условие взрезанного квадрата для изотопности строится следующим образом. Для изотопных вложений $f, g: N \rightarrow \mathbb{R}^{m}$ и изотопии $F: K \times I \rightarrow \mathbb{R}^{m}$ между ними определим отображение $\Phi: \widetilde{K} \times I \rightarrow S^{m-1}$ формулой

$$
\Phi(x, y, t)=\frac{F(x, t)-F(y, t)}{\|F(x, t)-F(y, t)\|}
$$

Это отображение является эквивариантной гомотопией между $\widetilde{f}$ и $\widetilde{g}$. Итак, необходимое условие взрезанного квадрата для изотопности вложений $f, g: K \rightarrow \mathbb{R}^{m}$ это эквивариантная гомотопность отображений $\widetilde{f}$ и $\widetilde{g}$. Это условие равносильно эквивалентности сечений расслоения $\widetilde{N} \times S^{m-1} /(t \times a) \underset{g}{\stackrel{S^{m-1}}{\longrightarrow}} \widetilde{N} / t[160]$, [147]. Заметим, что это условие (и его обобщение на изовариантные отображения или старшие взрезанные степени [50], [88]) не чувствует объемлемости изотопии, а также различия между DIFF, PL и TOP категориями. Поэтому оно (и его обобщения) не позволяет различить узлы в $\mathbb{R}^{3}$. Тем не менее, условие взрезанного квадрата очень хорошо работает в коразмерности $\geqslant 3$ (см. далее).

Необходимое условие взрезанного квадрата для погружаемости строится так. Для достаточно малой окрестности $O \Delta$ диагонали $\Delta$ в $N \times N$ обозначим $S N=O \Delta-\Delta$. Если $N$ - полиэдр, то эквивариантный гомотопический тип пространства $S N$ не зависит от $O \Delta$. Для погружения $h: N \rightarrow \mathbb{R}^{m}$ отображение $\widetilde{h}$ корректно определено на $S N$. Итак, необходимым условием взрезанного квадрата для погружсаемости $N$ в $\mathbb{R}^{m}$ назьвается существование эквивариантного отображения $S N \rightarrow S^{m-1}$. Необходимое условие взрезанного квадрата для регулярной гомотопности погружений $f$ и $g$ - это эквивариантная гомотопность отображений $\widetilde{f}$ и $\widetilde{g}$ на $S N$.

Итак, рассмотрим следующие утверждения для полиэдра (гладкого многообразия) $N$, обратные к которым только что были доказаны:

$($ EXI. $\varepsilon)$ Если сушествует эквивариантное отображение $\Phi: \widetilde{N} \rightarrow S^{m-1}$, то $N$ PL (DIFF) вложим (о) в $\mathbb{R}^{m}$.

(EXI.е) Если сушествует эквивариантное отображение $\Phi: \widetilde{N} \rightarrow S^{m-1}$, то сушествует $\mathrm{PL}(\mathrm{DIFF})$ вложение $f: N \rightarrow \mathbb{R}^{m}$ такое, что $\widetilde{f} \simeq$ еq $\Phi$.

(EXI.i) Если существует эквивариантное отображение $\Phi: S N \rightarrow S^{m-1}$, то сушествует $\mathrm{PL}(\mathrm{DIFF})$ погружение $h: N \rightarrow \mathbb{R}^{m}$ такое, что $\widetilde{h} \simeq$ еq $\Phi$ на $S N$.

(CLA.e) Если $f_{0}, f_{1}: N \rightarrow \mathbb{R}^{m}$ - два PL (DIFF) вложения и $\widetilde{f}_{0} \simeq$ eq $\tilde{f}_{1}$, то $f_{0}$ и $f_{1} \mathrm{PL}$ (DIFF) изотопны.

(CLA.i) Если $h_{0}, h_{1}: N \rightarrow \mathbb{R}^{m}$ - два PL (DIFF) погружения и $\widetilde{h}_{0} \simeq_{\text {еq }} \widetilde{h}_{1}$ на $S N$, то $h_{0}$ и $h_{1} \mathrm{PL}(\mathrm{DIFF})$ регулярно гомотопны.

Чтобы сформулировать теоремы достаточности для условий взрезанного квадрата, введем следуюшие обозначения. Прежде всего, положим $d=3 n-2 m+2$. Далее, 
всюду в этом параграфе мы будем опускать $\mathbb{Z}$-коэффициенты в обозначениях (ко)гомологических групп. Для $n \leqslant k+2$ через $\Sigma^{\infty}: \pi_{n+k}\left(S^{n}\right) \rightarrow \pi_{2 k+2}\left(S^{k+2}\right)=\pi_{k}^{S}$ обозначается стабильное отображение надстройки.

Tеорема 4.1. Для п-мерного полиәдра (соответственно DIFF многообразия) $N$ утверждения (ЕXI) верны при следующих условиях:

ع) $m-3 \geqslant n$ и $P L$ многообразие $N$ таково, что пара $(N, \partial N)$ является $\left[\frac{d}{3}\right]$-связной $и \pi_{1} \partial N=0$;

е) $m \geqslant \frac{3(n+1)}{2}\left(\right.$ либо $m \geqslant \frac{3(n+1)}{2}$, либо $(N, \partial N)(d-2)-$ связно, $\pi_{1} \partial N=0$ и $m \geqslant 6)$

i) $m \geqslant \frac{3(n+1)}{2}\left(\right.$ либо $m \geqslant \frac{3 n+1}{2}$, либо $(N, \partial N)$ гомологически $(d-2)$-связно $)$.

ТЕОрема 4.2. Если $N$ - n-полиәдр, то PL случаи утверждений (ЕXI) вернь при следующих условиях:

е) $N$ есть $P L$ многообразие и либо $m \geqslant n+3$ и $N$ замкнуто d-связно, либо $(N, \partial N)$ является $(d-1)$-связным,$\pi_{1} \partial N=0$ и $m \geqslant 6$.

i) либо $m \geqslant \frac{3 n}{2}+1$, либо $N$ есть $P L$ многообразие и $(N, \partial N)$ гомологически $(d-1)$-связно.

Для $d=1$ в замкнутом случае теоремы 4.2.е достаточно требовать лишш гомологической односвязности [137]. Теорема 4.1.ع - фольклорный результат (см. доказательство в [137]). Теорема 4.1.е доказана в [46; теоремы $1^{\prime}$ и 6.4], [153; теорема 1] (см. также [55], [136], [146]). Теорема 4.1.і доказана в [52], [55; теорема 2]. Теорема 4.2 доказана в [135], [137]. В этой работе будут доказаны только теорема 4.1.е для PL случая и $m \geqslant \frac{3(n+1)}{2}$, теорема 4.2 (и будет построен пример 4.5.ع, формулировку которого см. ниже).

Заметим, что теорема 4.2.е наиболее интересна для случая замкнутого $N$, поскольку в этом случае утверждение (EXI.i) не доказано (мы предполагаем, что оно просто неверно), и DIFF версия теоремы 4.2.е неверна (это вытекает из примеров $4.4 \varepsilon, \mathrm{e}^{\prime}$ ).

Интересное следствие работ [18], [19] выведено в [136]: континуум Пеано $K$ вложим в $\mathbb{R}^{2}$ тогда и только тогда, когда существует эквивариантное отображение $\widetilde{K} \rightarrow S^{1}$.

Из теории сглаживания [51; 1.6], [50; 11.1] следует, что если $N$ - некоторое DIFF многообразие, то к сглаживанию вложения в замкнутом случае теоремы 4.2.e имеется единственное препятствие, в $H^{n}\left(N, C_{n-1}^{m-n}\right)$. Поскольку $C_{8 k-2}^{4 k}=0$ [47; 8.15] (в $[47 ; 8.15]$ имеется опечатка: вместо $C_{4 k-2}^{3 k}=0$ должно быть $C_{8 k-2}^{4 k}=0$ ), то справедливо следующее утверждение.

СлЕДСТВИЕ 4.3. DIFF случай утверждения (ЕХI.е) (и его следствия) справедлив для гомологически 1-связного замкнутого DIFF $(8 l-1)$-многообразия $N$ $u m=12 l-1$.

Следствиями теорем 4.1.e, 4.2.e, 4.6.е и 4.7.е являются все результаты $\S 3$, кроме теоремы 3.2.b (хотя большинство из них может быть получено независимо) [42]. Из теорем 4.1.е и 4.2.е следует, что при их предположениях

1) PL (DIFF) вложимость $N$ в $\mathbb{R}^{m}$ не зависит от PL (DIFF) структур на $N$;

2) если $N$ ТОР вложимо в $\mathbb{R}^{m}$, то $N$ PL вложимо в $\mathbb{R}^{m}$;

3 ) если $N$ квазивложим в $\mathbb{R}^{m}$, то $N$ вложим в $\mathbb{R}^{m}$. 
Аналогичные следствия имеют теоремы 4.1.i, 4.2.i, 4.6, 4.7 (подробнее см. [137]).

Доказательство теоремы 4.2.e [137] не дает ее относительной и аппроксимационной версий (которые верны для теоремы 4.1.е [153; теоремы 7,3$]$ ). В отличие от этого доказательство случая $m \geqslant \frac{3 n}{2}+1$ теоремы 4.2.i [137] дает аппроксимационную версию (в которой построенное погружение сколь угодно близко к данному PL отображению $g$ ) и относительную версию (если $g: N \rightarrow \mathbb{R}^{m}$ - некоторое PL отображение, $A \subset N$ - подполиэдр и $\Phi: S N \rightarrow S^{m-1}$ - такое эквивариантное отображение, что $\left.g\right|_{A}$ - погружение и $\widetilde{g} \simeq$ еq $\Phi$ на $S A$, то существует погружение $h: N \rightarrow \mathbb{R}^{m}$ такое, что $h=g$ на $A$ и $\widetilde{h} \simeq_{\text {еq }} \Phi$ на $\left.S N\right)$. Для DIFF случая аппроксимационная версия верна, даже без предположения $m \geqslant \frac{3 n}{2}+1$, в следуюшей форме: если $N$ погружается в $\mathbb{R}^{m}$, то любое отображение $N \rightarrow \mathbb{R}^{m}$ аппроксимируется погружениями [39].

Очевидно, что условие связности $N$ может быть опущено из теоремы 4.2.е для $d=0$, если требовать вьполнения только условия (EXI. $\varepsilon$ ), а не условия (ЕXI.e). Для $m<\frac{5 n+6}{4}$ теорема 4.2.е не интересна: имеем $d>\frac{n}{2}-1$ и $n \geqslant 6$, значит $N-$ гомотопическая сфера, поэтому $N \cong S^{n}$ и теорема 4.2.е верна (ср. с замечанием после теоремы 3.2). Но доказательство теоремы 4.2.е не упрошается при $m \geqslant \frac{5 n+6}{4}$, и оно также может рассматриваться как шаг к доказательству аналога теоремы 4.2.е для вложений в многообразия.

ПримеP 4.4. $\varepsilon$ ) DIFF случай утверждения (EXI. $\varepsilon$ ) не верен для $(m, n)=(19,16)$ и гомотопической сферы $N$.

е) Если $l \geqslant 3$ и $\Sigma^{\infty}: \pi_{q}\left(S^{l}\right) \rightarrow \pi_{q-l}^{S}-$ не эпиморфизм, то ни PL, ни DIFF случаи утверждения (EXI.e) не верны для несвязного многообразия $N=S^{q} \sqcup S^{q}$ и $m=$ $q+l+1$.

$\mathrm{e}^{\prime}$ ) DIFF случай утверждения (ЕXI.е) (даже теоремы 3.6.2) не верен для замкнутого (даже $(2 n-m)$-связного) многообразия $N=S^{2 k} \times S^{2 k}$ и $m=\frac{3 n}{2}+1=6 k+1$.

ПримеР 4.5. $\varepsilon$ ) Полиэдральньй случай утверждения (ЕХI. $\varepsilon$ ) не верен для любой пары $(m, n)$ такой, что $4 \leqslant m \leqslant \frac{3 n}{2}+1$.

е) Если $l \geqslant 3$ и $\Sigma_{[l-1]}^{\infty}: \pi_{q}\left(S^{l}\right)_{[l-1]} \rightarrow \pi_{q-l,[l-1]}^{S}-$ не эпиморфизм, то ни PL, ни DIFF случаи утверждения (ЕXI.е) не верны для $N=S^{1} \times S^{q}$ и $m=q+l+1$.

$\left.\mathrm{e}^{\prime}\right)$ Если $l \geqslant 2, \Sigma_{(l)}^{\infty}: \pi_{q}\left(S^{l}\right)_{(l)} \rightarrow \pi_{q-l,(l)}^{S}-$ не эпиморфизм и $\Sigma^{\infty}: \pi_{q}\left(S^{l+1}\right) \rightarrow \pi_{q-l-1}^{S}$ - мономорфизм, то ни PL, ни DIFF случаи утверждения (EXI.e) не верны для $N=$ $S^{1} \times S^{q}$ и $m=q+l+2$.

$\mathrm{e}^{\prime \prime}$ ) 3 -адический соленоид $\Sigma$ (т.е. пересечение бесконечной последовательности полноториев, каждый из которых вписан в предыдуший со степенью 3 ) не вложим в $\mathbb{R}^{2}$, хотя существует эквивариантное отображение $\widetilde{\Sigma} \rightarrow S^{1}$.

$\mathrm{e}^{\prime \prime \prime}$ ) (Гипотеза) Существует непланарньй древовидный континуум $K$, для которого имеется эквивариантное отображение $\widetilde{K} \rightarrow S^{1}$.

i) PL случай утверждения (EXI.i) не верен для любых $5 \leqslant m \leqslant \frac{3 n+1}{2}$ или $m=$ $n=4$.

Пример 4.4.є построен в [61] (см. также [109], [117]). Утверждение примера 4.4.е следует из [47]. Утверждение примера 4.4.e' следует из [8; теоремы 1.5 и 1.6], [7; теорема 4.2], доказательство см. в [137]. Пример 4.5.є построен в [29], [77], [99], [130], [131]. Примеры 4.5.e,e', і построены в [137]. Пример 4.5.e ${ }^{\prime \prime}$ построен в [136], ср. с [119; пример 1.5]. 
Примеры 4.5.e, $\mathrm{e}^{\prime}$ показывают, что предположение связности в замкнутом случае теоремы 4.2.е не может быть существенно ослаблено. Действительно, из них и таблиц в [148] следует, что утверждение (EXI.е) неверно для $N=S^{q} \sqcup S^{q}, q=6,14$ и $m=$ $\frac{3 q}{2}+1$ (пример 4.4.е) и для $N=S^{1} \times S^{q}$ в следующих случаях (первые 7 столбцов получены из примера 4.5.e, а последние 3 - из примера 4.5.e'; см. также [137]):

$$
\begin{aligned}
& q=\begin{array}{llllllllll}
6 & 14 & 12 & 13 & 26 & 29 & 28 & 13 & 11 & 12
\end{array} \\
& \begin{array}{llllllllll}
m= & 10 & 22 & 18 & 19 & 38 & 43 & 40 & 21 & 17
\end{array} \\
& m=\frac{3 n-1}{2} \quad \frac{3 n-1}{2} \quad \frac{3 n-3}{2} \quad \frac{3 n-4}{2} \quad \frac{3 n-5}{2} \quad \frac{3 n-4}{2} \quad \frac{3 n-7}{2} \quad \frac{3 n}{2} \quad \frac{3 n-2}{2} \quad \frac{3 n-3}{2}
\end{aligned}
$$

Сформулируем теперь классификационные версии вышеизложенных результатов.

ТЕОрема 4.6. Если $N$ - некоторый $n$-полиәдр (DIFF $n$-многообразие), то утверждения (CLA) верны при следующих условиях:

е) $m \geqslant \frac{3 n}{2}+2\left(\right.$ либо $m \geqslant \frac{3 n}{2}+2$, либо пара $(N, \partial N)$ является $(d-1)$-связной, $\left.\pi_{1} \partial N=0 u m \geqslant 7\right)$

i) $m \geqslant \frac{3 n}{2}+2$ (либо $m \geqslant \frac{3 n}{2}+1$, либо пара $(N, \partial N)$ гомологически $(d-1)$ связна).

ТЕОРема 4.7. Если $N$ - некоторый n-полиэдр, то PL случаи утвержсдений (CLA) верны при следующих условиях:

е) $N$ является $P L$ многообразием, $m \geqslant n+3$ и либо $N$ замкнуто $u(d+1)$ связно, либо пара $(N, \partial N)$ является $d$-связной $и \pi_{1} \partial N=0$;

i) либо $m \geqslant \frac{3(n+1)}{2}$, либо $N$ является $P L$ многообразием и пара $(N, \partial N)$ гомологически $d$-связна.

Для $d=0$ в теореме 4.7 достаточно требовать лишь гомологической односвязности. Теорема 4.6.е доказана в [46; теорема $1^{\prime}$ ], [156; теорема $\left.1^{\prime}\right]$, см. также [55; следствие 1], [135; § 3]. Теорема 4.6.і доказана в [52], [55; сноска на с. 3]. Теорема 4.7 доказана в $[135],[137]$.

Интересное следствие работы [103] было доказано в [160] для графов и в [136] для обшего случая: вложения $f, g: K \rightarrow \mathbb{R}^{2}$ континуума Пеано $K$ изотопны тогда и только тогда, когда $\widetilde{f} \simeq$ eq $\widetilde{g}$.

Такие же замечания, как о сглаживании вложения в теореме 4.2.e, о предположении связности в теореме 4.2.е и об относительной и аппроксимационной версиях теоремы 4.2, можно сделать для теоремы 4.7.е, теоремы 4.7.е и теоремы 4.7 соответственно.

СлЕДСТВИЕ 4.8. 1) DIFF случай утверждения (CLA.e) справедлив для гомологически односвязного замкнутого DIFF $(8 l-2)$-многообразия $N$ u $m=12 l-2$.

2) Если $p \leqslant q u m \geqslant \frac{3 q}{2}+p+2\left(m \geqslant \frac{3(q+p)}{2}+2\right)$, то множество $P L$ $(D I F F)$ вложений $S^{p} \times S^{q} \rightarrow \mathbb{R}^{m}$ с точностью до $P L(D I F F)$ изотопии находится во взаимно однозначном соответствии с множеством әлементов группь $\pi_{q}\left(V_{m-q, p+1}\right) \oplus \pi_{p}\left(V_{m-p, q+1}\right)$.

Следствие 4.8 доказано в [137] (для $m=2 q+p+1$ следствие 4.8.2 доказано в [64]). О вычислениях групп $\pi_{r}\left(V_{m n}\right)$ см. [79], [111], [155]. 
ПримеР 4.9. е) PL случай утверждения (CLA.e) неверен для несвязного многообразия $N=S^{n} \sqcup S^{n} \sqcup S^{n}$ и $m=\frac{3(n+1)}{2}$.

eie) DIFF случай утверждения (CLA.e) (и теоремы 11.1.a) не верны для $m=\frac{3(n+1)}{2}$ и $N=S^{n}$ (которое, конечно, $(d+1)$-связно).

$\left.\mathrm{e}^{\prime}\right)$ PL случай утверждения (CLA.e) неверен для любой пары $(m, n)$ такой, что $n+3 \leqslant m \leqslant \frac{3(n+1)}{2}$, но $m-n \notin\{4,8\}$, и полиэдра (для $m=\frac{3(n+1)}{2}$, несвязного PL многообразия) $N=S^{n} \sqcup S^{2 m-2 n-3}$.

i) Случай PL (DIFF) многообразий утверждения (CLA.i) неверен для $m=\frac{3 n}{2}+1$ $\left(m=\frac{3 n+1}{2}\right)$ и $N=S^{n}$.

ПримеР 4.10. е) Для любого целого $l=2$ или $l \geqslant 4$ если $\Sigma^{3}: \pi_{4 l+1}\left(S^{2 l}\right) \rightarrow \pi_{2 l+1}^{S}$ - эпиморфизм, то ни PL, ни DIFF случай утверждения (CLA.e) не верны для $N=$ $S^{1} \times S^{4 l+1}$ и $m=6 l+3=\frac{3 n}{2}$.

$\left.\mathrm{e}^{\prime}\right) \mathrm{PL}$ случай утверждения (CLA.e) неверен для любой пары $(m, n)$ такой, что $n+2 \leqslant m \leqslant \frac{3(n+1)}{2}$, и полиэдра $N=S^{n} \vee S^{n} \sqcup S^{2 m-2 n-3}$.

Пример 4.9.е построен в [45], [102; утверждение 8.3]. Пример 4.9.еіе построен в [43], [47; 8.14]. Пример 4.9.e' следует из [45], [47] (доказательство см. в [137]). Пример 4.9.і следует из [50], [49] (доказательство см. в [137]). Пример 4.10 построен в [137].

Пример 4.10.е показывает, что предположение связности в теореме 4.7.е не может быть сушественно ослаблено: (CLA.е) верно для $m \geqslant \frac{3 q}{2}+p+2$, но может быть неверно уже для $m=\frac{3 q+3}{2}$. Предположения примера 4.10.е выполнены для всех $l \leqslant 15$, и мы высказываем гипотезу, что они выполнены для всех $l=2$ или $l \geqslant 4$. Мы высказываем гипотезу, что PL (DIFF) случай утверждения (EXI.i) неверен для PL (DIFF) многообразий и $m=\frac{3 n+1}{2}\left(m=\frac{3 n}{2}\right)$.

Пример с кольцами Борромео в начале $\S 6$ (ср. [120]) наводит на мысль, что можно построить препятствия к вложимости (или относительной вложимости), аналогичные препятствиям Ван Кампена и взрезанного квадрата, но полученные из трехкратных (четырехкратных, . . . ) пересечений. Более того, отсутствие этих препятствий будет достаточным для вложимости, даже когда для препятствий Ван Кампена и взрезанного квадрата это не так. Возможньй кандидат на необходимое условие к вложимости для случая, когда условие взрезанного квадрата не является достаточньп, это условие $G$-взрезанной степени. Оно формулируется следующим образом. Пусть $G$ - подгруппа групшы $S_{p}$ подстановок из $p$ элементов и

$$
\widetilde{N}_{G}=\left\{\left(x_{1}, \ldots, x_{p}\right) \in N^{p} \mid x_{i} \neq x_{\sigma(i)} \text { для всех } \sigma \in G, i=1, \ldots, p\right\} .
$$

Пространство $\widetilde{N}_{G}$ называется $G$-взрезанной степенъю пространства $N$. На пространстве $\widetilde{N}_{G}$ очевидным образом действует группа $G$. Если $f: N \rightarrow \mathbb{R}^{m}$ - отображение, то формула $\widetilde{f}_{G}\left(x_{1}, \ldots, x_{p}\right)=\left(f x_{1}, \ldots, f x_{p}\right)$ корректно определяет отображение $\left.\tilde{f}_{G}: \widetilde{N}_{G} \rightarrow \widetilde{\left(\mathbb{R}^{m}\right.}\right)_{G}$. Ясно, что отображение $\widetilde{f}_{G}$ является $G$-эквивариантным. Итак, необходимым условием $G$-взрезанной степени для вложимости $N$ в $\mathbb{R}^{m}$ называется существование некоторого $G$-эквивариантного отображения $\Phi: \widetilde{N}_{G} \rightarrow \widehat{\left(\mathbb{R}^{m}\right)_{G}}$. Заметим, что это условие хорошо работает в теории сингулярных зацеплений [102]. В отличие от этого примеры 4.4 и 4.5. $\varepsilon$ показьвают, что не только условие взрезанного квадрата, но даже условие $G$-взрезанной степени (с любой группой $G$ ) не являются достаточными для вложимости. 
Аналогично определяются условия $G$-взрезанной степени для погружаемости, изотопности и регулярной гомотопности. Предположительно, пример 4.5.і показывает, что условие $G$-взрезанной степени не является достаточным для погружаемости. Примеры 4.9.eie, $\mathrm{e}^{\prime}$ и 4.10.е $\mathrm{e}^{\prime}$ показьвают недостаточность условия $G$-взрезанной степени (с любой группой $G$ ) для изотопности. Заметим, что пример 4.9.е для этого не годится. Пример 4.9.і показывает, что условие $G$-взрезанной степени (с любой группой $G$ ) не является достаточным для регулярной гомотопности.

\section{§. Приложение: базисные вложения в плоскость}

В работах, посвященных решению 13-й проблемы Гильберта, А. Н. Колмогоров [87] и В.И. Арнольд [6] доказали, что любая непрерывная функция $n$ аргументов, определенная на компактном подмножестве $\mathbb{R}^{n}$, может быть представлена как суперпозиция непрерьвных функций одного аргумента и сложения (популярное изложение см. в [5]). Остранд обобшил эту теорему на произвольные $n$-мерные компакты [110]. В этих работах фактически появилось понятие базисного вложения, явно введенное в [144]. Вложение $K \subset \mathbb{R}^{m}$ называется базисным, если для любой непрерывной функции $f: K \rightarrow \mathbb{R}$ существуют непрерьвные функции $g_{1}, \ldots, g_{m}: \mathbb{R} \rightarrow \mathbb{R}$ такие, что $f\left(x_{1}, \ldots, x_{m}\right)=g_{1}\left(x_{1}\right)+\cdots+g_{m}\left(x_{m}\right)$ для любой точки $\left(x_{1}, \ldots, x_{m}\right) \in K$.

Tеорема 5.1 [87], [6], [110], [145], [94]. Любой п-мерный компакт базисно вложим в $\mathbb{R}^{2 n+1}$ и при $n>1$ не вложим базисно в $\mathbb{R}^{2 n}$.

Интересно сравнить эту теорему с теоремой Нёбелинга-Менгера-Понтрягина о том, что любой $n$-мерньй компакт вложим в $\mathbb{R}^{2 n+1}[76]$, и примером $n$-мерного полиэдра, не вложимого в $\mathbb{R}^{2 n}[28]$, [80]. Очевидно, что $K$ базисно вложим в $\mathbb{R}$ тогда и только тогда, когда $K$ топологически вложим в $\mathbb{R}$. Из теоремы 5.1 следует, что для $m>2$ компакт $K$ базисно вложим в $\mathbb{R}^{m}$ тогда и только тогда, когда $\operatorname{dim} K<m / 2$. Итак, единственным неразобранным случаем остается случай $m=2$. Проблема характеризации компактов, базисно вло женных в плоскость, была поставлена еще в [5] и решена в [145]: компакт $K$ базисно вложен в плоскость тогда и только тогда, когда $E^{n}(K)=\varnothing$ для некоторого $n$. Здесь

$$
E(Z)=\left\{z \in Z:\left|Z \cap p_{x}^{-1} p_{x} z\right| \geqslant 2 \text { и }\left|Z \cap p_{y}^{-1} p_{y} z\right| \geqslant 2\right\}
$$

a $p_{x}$ и $p_{y}$ - проекции на координатные оси в плоскости. Характеризацию линейно-связных компактов, базисно вложимы в плоскость, полученную в [134], удобно сформулировать сначала для графов и затем для общего случая. Гипотезу о вложимости (не обязательно линейно-связных) континуумов в плоскость см. в [134].

Teорема 5.2 ([134]; ср. [89], [98], [118; §2]). Конечный граф базисно вложим в плоскость тогда и только тогда, когда выполнено любое из следующих двух эквивалентных условий:

а) он не содержит подграфов, гомеоморфньх $S, C_{1}, C_{2}$ (рис. 5.1.а) - окружности, звезды с 5 лучами и креста с разветвленными концами;

b) он содержится в одном из графов $V_{n}$ (рис. 5.1.b). 

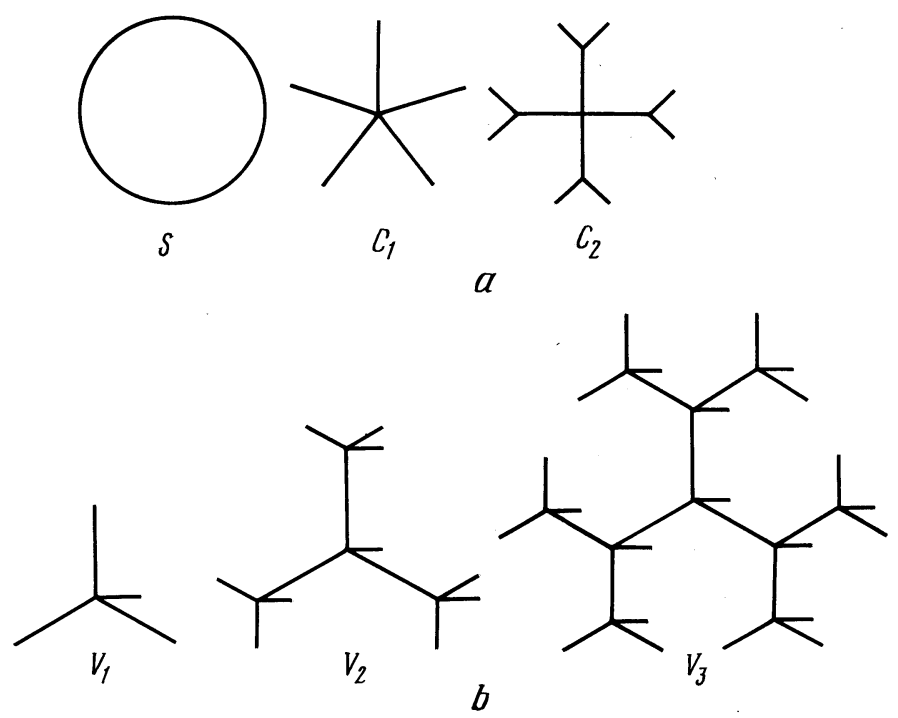

Рис. 5.1

Teорема 5.3 ([90]; см. также [15], [91]). Для конечного графа $K$ существует базисное вложсние $K \subset \mathbb{R} \times T_{n}$ (т.е. вложение такое, что для любой непрерьвной функиии $f: K \rightarrow \mathbb{R}$ существуют непрерьвные функиии $g: \mathbb{R} \rightarrow \mathbb{R}, h: T_{n} \rightarrow \mathbb{R}$ такие, что $f(x, y)=g(x)+h(y)$ для любой точки $(x, y) \in K)$ тогда и только тогда, когда $K$ - дерево и либо $\delta(K)<n$, либо $[\delta(K)=n$ и $K$ содерэмит ужасную вершину, имеющую висячее ребро].

Здесь $T_{i}-i$-од, т.е. звезда с $i$ лучами. Вершина графа $K$ называется ужсасной, если ее степень больше четырех. Вершина графа $K$ назьвается страшной, если ее степень равна четырем и она не является концом ни одного висячего ребра. Дефектом графа $K$ назьвается сумма $\delta(K)=\left(\operatorname{deg} A_{1}-2\right)+\cdots+\left(\operatorname{deg} A_{k}-2\right)$, где $A_{1}, \ldots, A_{k}-$ все страшные и ужасные вершины графа $K$.

Чтобы охарактеризовать линейно связные континуумы, базисно вложимые в плоскость, введем некоторые обозначения и определения. Через I обозначается отрезок $[0,1]$. Если $J$ - дуга, то через Int $J$ обозначается $J$ без концов. Дуга $s$ называется соединяющей точки $A$ и $B$, если $s \cap A$ и $s \cap B$ - различные концы $s$. Hyль-nоследовательностью множеств назьвается последовательность множеств, диаметры которых стремятся к нулю. $C_{3}-$ крест с нуль-последовательностью дуг, сходящихся к его центру и приклеенных к одной из его ветвей (рис. 5.2). $C_{4}$ - крест с последовательностью точек, сходящихся к его центру (рис. 5.3). $B$ - объединение дуги $I$ и нуль-последовательности дуг, приклеенных за один конец к $\stackrel{\circ}{I}$ (рис. 5.3). Очевидно, что топологический тип $B$ не зависит от вариаций в его построении. $F_{1}$ - триод, и $F_{n+1}$ получено из $F_{n}$ разветвлением каждого конца $F_{n}$ (рис. 5.3 ). $H_{n}$ - объединение $I$ с нуль-последовательностью триодов, приклеенных к $I$ за один конец в точках множества $D_{n}=\left\{3^{-l_{1}}+\cdots+3^{-l_{s}} \mid s \leqslant n, 0<l_{1}<\cdots<l_{s}\right.$ - целые $\}$ (рис. 5.3). $F$ - объединение $I$ с нуль-последовательностью множеств $F_{n}$, приклеенных к точкам $1 / n \in I$ за один конец (рис. 5.2). $H_{+}$и $H_{-}$- объединения $I$ с нуль-последователь- 

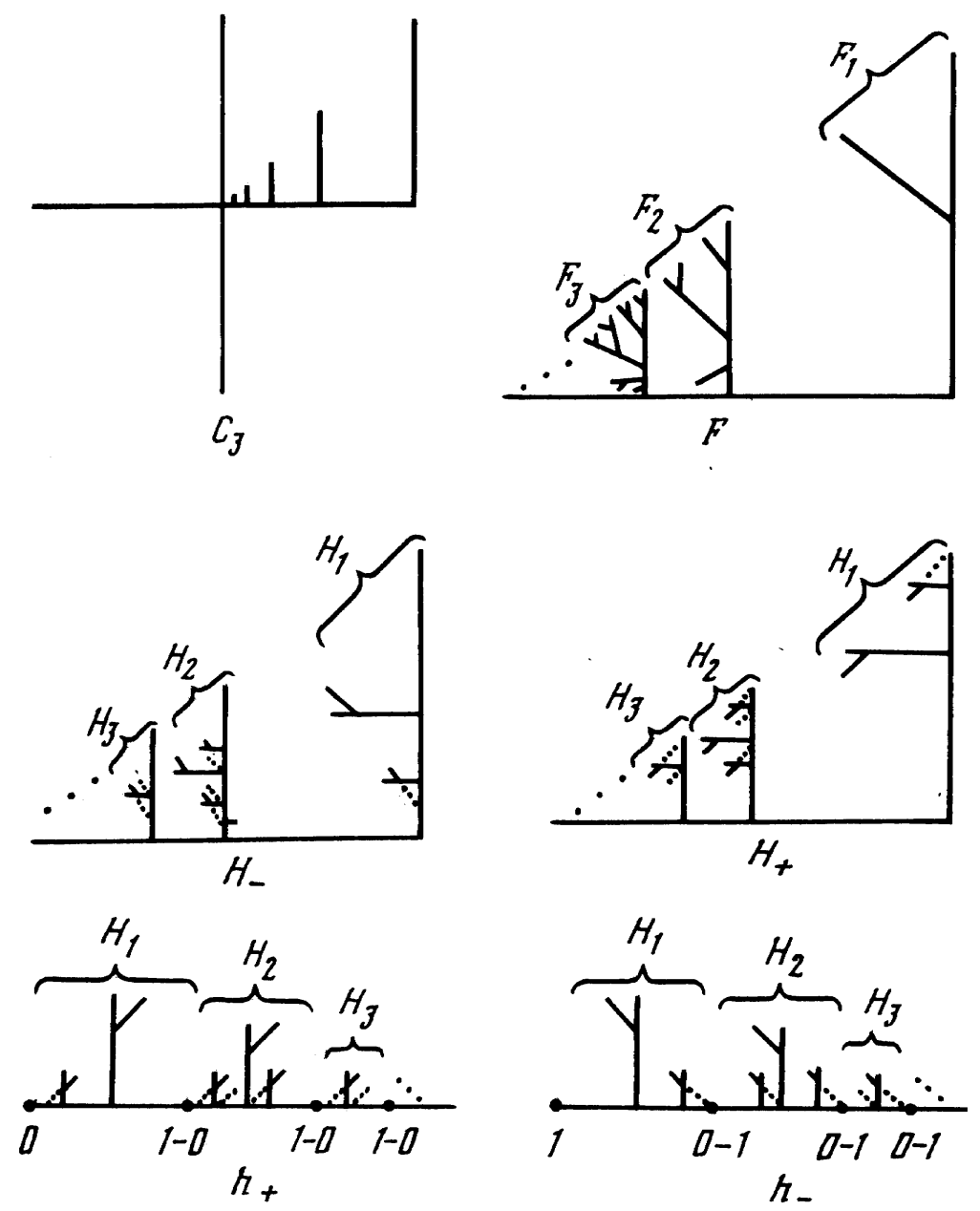

Рис. 5.2

ностью континуумов $H_{n}$, соединенные с точками $1 / n \in I$ дугами, пересекаюшими $H_{n}$ в $1 \in I \subset H_{n}$ и $0 \in I \subset H_{n-1}$ соответственно (рис. 5.2). $h_{+}\left(h_{-}\right)$получено из нуль-последовательности континуумов $H_{n}$ склеиванием точек $1 \in I \subset H_{n}$ и $0 \in I \subset H_{n-1}$ (соответственно $0 \in I \subset H_{n}$ и $1 \in I \subset H_{n-1}$ ) (рис. 5.2). Фильтрацией назьвается последовательность $I_{1} \subset I_{2} \subset \cdots \subset I_{n}=K$ такая, что $I_{n+1}$ получено из $I_{n}$ приклеиванием нуль-последовательности дуг за один конец каждой. Назовем эти дуги дугами порядка l.

TеОРема 5.4 [134]. Линейно связный компакт К базисно вложим в плоскость тогда и только тогда, когда он является локально связным (т.е. пеановским) и выполнено любое из трех следующих (эквивалентных) условий:

1) $K$ не содержит подкомпактов $S, C_{2}, C_{4}, B$ и содержит лишь конечное число подконтинуумов $F_{n}, H_{n}$ (см. рис. 5.1, 5.3);

2) (ср. [18], [19]) $K$ не содерэит подконтинуумов $S, C_{1}, C_{2}, C_{3}, B, F, H_{+}, H_{-}$, 


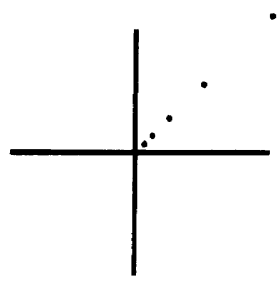

$c_{4}$

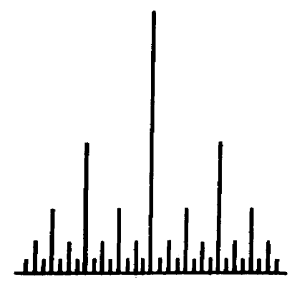

$B$

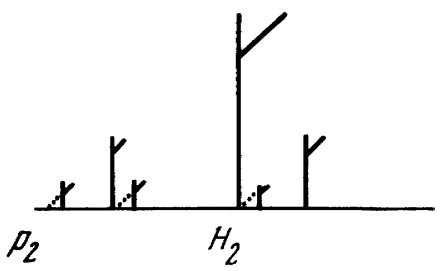

Рис. 5.3

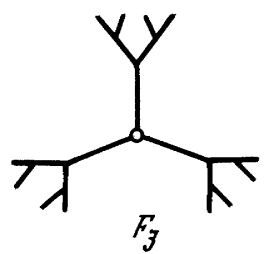

$F_{3}$
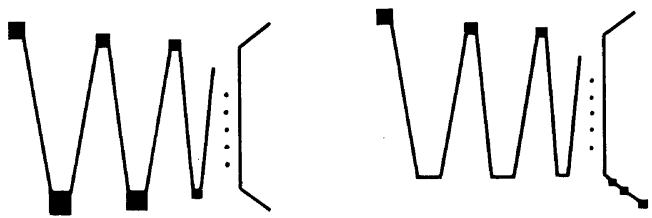

Рис. 5.4

$h_{+}, h_{-}($puc. 5.2, 5.3);

3) существуют фильтрация $I_{1} \subset \cdots \subset I_{n}=K$ и натуральное число $M$ такие, что для любой дуги $s$ порядка $l-1\left(s=I_{1}\right.$, когда $\left.l=1\right)$, обозначая через $\left\{s_{m}\right\}$ все дуги порядка не менее $l$, пересекающие $s, u R=s \cap\left(\bigcup_{m} s_{m}\right)$, имеем:

I) $R \subset \operatorname{Int} s$;

C) к каждой точке $s$ приклеено не более двух дуг $s_{m}$, и если ровно две, то одна из них - висячая (т.е. ни одна дуга порядка не менее $l+1$ не пересекает ее), причем точка, $к$ которой приклеены эти две дуги, имеет окрестность в $K$, гомеоморфную кресту;

B) $\mathrm{Cl} R$ нигде не плотно в $S$;

H) $h^{M} Q=\varnothing$, где $Q=\left\{x \in R \mid\right.$ если $x \in s_{m}$, то $s_{m}-$ не висячая $\}$ и $h Z-$ множество, полученное из $Z$ выкидыванием изолированных в $Z$ точек.

\section{II. Исследование полноты препятствия взрезанного квадрата}

\section{Обозначения}

Мы используем обозначения из [125]. Верхний индекс полиэдра указьвает его размерность. Симплексы любой триангуляции $T$ считаются линейно упорядоченными по 
возрастанию размерности. На $T \times T$ рассматривается лексикографический порядок. Для отображения $f: N \rightarrow \mathbb{R}^{m}$ через

$$
\widetilde{\Delta}(f)=\{(x, y) \in N \times N \mid x \neq y, f x=f y\} \quad \text { и } \Sigma(f)=\left\{x \in N:\left|f^{-1} f x\right| \geqslant 2\right\}
$$

обозначим его сингулярное множество. Строгое определение регулярной окрестности дано в $\S 8$ (после доказательства PL случая теоремы 3.1), однако для понимания идей приводимых доказательств (в частности, перед § 8) достаточно представлять себе регулярную окрестность как достаточно малую окрестность без “лишних дыр". Через $\operatorname{link}(\cdot, \cdot)$ мы обозначаем коэффициент зацепления.

\section{$\S$ 6. Построение примера 4.5. $\varepsilon$}

В этом параграфе $\widetilde{P}$ обозначает не взрезанньй квадрат пространства $P$, а копию пространства $P$ (для подмножества $A \subset P$ его копия обозначается через $\widetilde{A} \subset \widetilde{P}$ ).

Проиллюстрируем одну из основных идей построения примера Фридмана-Крушкаля-Тайхнера (теорема 2.1.а для $n=2$ и пример 4.5. $\varepsilon$ для $m=4, n=2$ ) [29]. Для этого построим три окружности, вложенные в $\mathbb{R}^{3}$, так что каж дые две из них не зацеплены, но все три вместе зацеплены. Будем называть такие тройки окружностей в $\mathbb{R}^{3}$ обобщенными кольцами Борромео. Наше построение основано на том, что фундаментальная группа не всегда коммутативна. Возьмем две незаузленные окружности $\Sigma$ и $\widetilde{\Sigma}$ в $\mathbb{R}^{3}$ далеко друг от друга. Вложим в $\mathbb{R}^{3}-(\Sigma \sqcup \widetilde{\Sigma})$ “восьмерку", т.е. букет двух окружностей $C$ так, что включение $C \subset \mathbb{R}^{3}-(\Sigma \sqcup \widetilde{\Sigma})$ индуцирует изоморфизм фундаментальных групп. Возьмем образующие $a$ и $b$ из $\pi_{1}(C)=\pi_{1}\left(\mathbb{R}^{3}-(\Sigma \sqcup \widetilde{\Sigma})\right)$, представленные двумя произвольно ориентированньми окружностями "восьмерки". Рассмотрим отображение $S^{1} \rightarrow C \subset \mathbb{R}^{3}$, представляющее элемент $a b a^{-1} b^{-1}$. Используя соображения общего положения, построим вложсение $f: S^{1} \rightarrow \mathbb{R}^{3}$, очень близкое к этому отображению. Тогда $\Sigma, \widetilde{\Sigma}$ и $f\left(S^{1}\right)$ суть обобщенные кольца Борромео. Действительно, $\Sigma$ и $\widetilde{\Sigma}$ не зацеплены по определению. Легко выбрать $f$ так, что $\Sigma$ и $f\left(S^{1}\right)$, а также $\widetilde{\Sigma}$ и $f\left(S^{1}\right)$ не зацеплены (поскольку $f$ индуцирует нулевой гомоморфизм одномерных групп гомологий). Но $f$ индуцирует ненулевой гомоморфизм фундаментальных групп. Поэтому $\Sigma, \widetilde{\Sigma}$ и $f\left(S^{1}\right)$ вместе зацеплены.

Из сушествования обобшенных колец Борромео можно вывести следующий контрпример к относительному случаю теоремы 2.1.а для $n=2$. Пусть $K=D^{2} \sqcup D^{2} \sqcup D^{2}$, $A=\partial D^{2} \sqcup \partial D^{2} \sqcup \partial D^{2}$ и $A \subset S^{3} \cong \partial D^{4}$ - обобщенные кольца Борромео. Так как все три кольца зацеплены, то вложение $A \rightarrow \partial D^{4}$ не может быть продолжено до вложения $K \rightarrow D^{4}$. Но незацепленность каждой пары колец Борромео влечет обнуление соответствующего препятствия Ван Кампена для этого продолжения. Это понятно, так как препятствие Ван Кампена учитывает двукратные пересечения, но не учитывает трехкратные.

Теперь мы можем построить пример Фридмана-Крушкаля-Тайхнера. Пусть $P$ это 2-остов 6-симплекса без внутренности некоторого 2-симплекса из указанного 2-остова. Вспомним (третий абзац $\S 2$ ), что $P$ содержит две непересекающиеся сферы $\Sigma^{2}, \Sigma^{1}$ такие, что для любого вложения $P \rightarrow \mathbb{R}^{4}$ эти сферы зацеплены с ненулевым (в действительности, равным \pm 1 ) коэффициентом зацепления. Вложим $P$ и $\widetilde{P}$ в $\mathbb{R}^{4}$ стандартно (т.е. так, что $\Sigma^{2}$ и $\widetilde{\Sigma}^{2}$ не заузлены, а пары $\Sigma^{2}$ и $\Sigma^{1}, \widetilde{\Sigma}^{2}$ и $\widetilde{\Sigma}^{1}$ - стандартно зацепленные сферы) и далеко друг от друга. Тогда $\Sigma^{2}$ и $\widetilde{\Sigma}^{2}$ не зацеплены. Возьмем 
любую точку $x \in \Sigma^{1}$, соединим точки $x$ и $\widetilde{x}$ дугой и притянем эти точки друг к другу вдоль этой дуги (рис. 6.1). Получим вложение $P \vee \widetilde{P} \subset \mathbb{R}^{4}$. Пусть $C=\Sigma^{1} \vee \widetilde{\Sigma}^{1}-$ "восьмерка" (с отмеченной точкой $x=\widetilde{x})$. Тогда включение $C \subset \mathbb{R}^{4}-\left(\Sigma^{2} \sqcup \widetilde{\Sigma}^{2}\right)$ индуцирует изоморфизм фундаментальных групп. Выберем образующие $a$ и $b$ группы $\pi_{1}(C)$, представленные двумя (произвольно ориентированньми) окружностями "восьмерки". Возьмем отображение $h: S^{1} \rightarrow C$, представляющее элемент $a b a^{-1} b^{-1}$. Пусть $K-$ конус композищии $h$ с включением $C \subset P \vee \widetilde{P}$ (т.е. $K=D^{2} \cup_{h: \partial D^{2} \rightarrow C}$ $(P \vee \widetilde{P}))$.

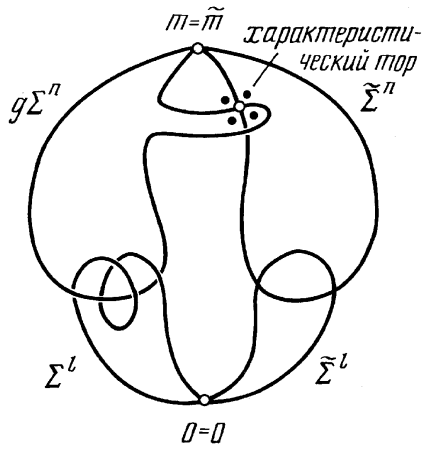

Рис. 6.1

Тогда $K$ не вложим в $\mathbb{R}^{4}$, хотя $v(K)=0$. Подробное доказательство можно найти в [29]. Причина равенства $v(K)=0$ в том, что препятствие Ван Кампена учитывает гомологическое свойство (то, что цикл $a b a^{-1} b^{-1}$ гомологичен нулю) и не учитывает более тонкое гомотопическое свойство (то, что цикл $a b a^{-1} b^{-1}$ не гомотопен нулю). Сделаем набросок доказательства невложимости $K$ в $\mathbb{R}^{4}$. Предположим противное, что существует вложение $g: K \rightarrow \mathbb{R}^{4}$. Если $g \Sigma^{2}$ и $g \widetilde{\Sigma}^{2}$ незаузлены в $\mathbb{R}^{4}$, то из свойств полиэдра $P$ следует, что отображение $C \rightarrow g C \subset \mathbb{R}^{4}-g\left(\Sigma^{2} \sqcup \widetilde{\Sigma}^{2}\right)$ индуцирует мономорфизм фундаментальных групп. В общем случае это доказывается с помощью теоремы Столлингса о нижнем центральном ряде группы [143], [29]. Но элемент $a b a^{-1} b^{-1}$, который не равен нулю в $\pi_{1} C$, переходит в петлю в $\mathbb{R}^{4}-g\left(\Sigma^{2} \sqcup \widetilde{\Sigma}^{2}\right)$, которая продолжается до $g D^{2}$ и, следовательно, гомотопна нулю. Полученное противоречие доказьвает невложимость $K$ в $\mathbb{R}^{4}$.

Приведем теперь построение примера 4.5.ع, являющееся многомерным обобшением описанного выше примера Фридмана-Крушкаля-Тайхнера. Полиэдр $K$ назьвается квазивложимым в $\mathbb{R}^{m}$, если для каждой его триангуляции $T$ сушествует отображение $f: K \rightarrow \mathbb{R}^{m}$ (которое назьвается почти вложением) такое, что $f \sigma \cap f \tau=\varnothing$ для любых $\sigma \times \tau \in \widetilde{T}$. Это определение нестандартно, но эквивалентно стандартному $(\S 1)$. Достаточно построить $n$-полиэдр $R$, квазивложимый в $\mathbb{R}^{m}$, но не вложимьй в $\mathbb{R}^{m}$. Пусть $l=m-n-1$. Достаточно построить пример 4.5. $\varepsilon$ для $m=n+2 \geqslant 4$ и для $n+3 \leqslant m \leqslant \frac{3 n}{2}+1$.

ЛЕмма 6.1 (ср. [29; лемма 6], [131; лемма 1.4]). Для любъх $l \geqslant \frac{n}{2}$ существует $n$-полиэдр K, содержащий два непересекающихся букета сфер $\Sigma^{n} \vee \widetilde{\Sigma}^{n} u \Sigma^{l} \vee \widetilde{\Sigma}^{l}$ 
maкux, чmo

а) для любого $P L$ вложения $K \subset \mathbb{R}^{m}$ пары $\Sigma^{n}, \widetilde{\Sigma}^{l}$ и $\widetilde{\Sigma}^{n}, \Sigma^{l}$ незачеплены и гомологический коэффичиент зачепления пар $\Sigma^{n}, \Sigma^{l}$ и $\widetilde{\Sigma}^{n}, \widetilde{\Sigma}^{l}$ не равен нулю (для $m=n+2$ этот коэффициент нечетен);

b) существует $P L$ вложение $K \subset \mathbb{R}^{m}$, для которого букет $\Sigma^{n} \vee \widetilde{\Sigma}^{n}$ незаузлен $в \mathbb{R}^{m}$.

ДокАЗАТЕльство. Обозначим через $\Delta_{a_{0} \ldots a_{s}}^{k} k$-мерньй остов $s$-мерного симплекса с вершинами $a_{0} \ldots a_{s}$. Пусть

$P=\Delta_{12 \ldots m+2}^{n} \cup \operatorname{Con}\left(\Delta_{12 \ldots m+2}^{l}, 0\right) \quad$ и $Q=\Delta_{12 \ldots m+2}^{n} \cup \operatorname{Con}\left(\Delta_{12 \ldots m+2}^{l}-\stackrel{\circ}{\Delta}_{12 \ldots l+1}^{l}, 0\right)$.

Пусть $\Sigma^{l}=\partial \Delta_{01 \ldots l+1}^{l+1}, \Sigma^{n}=\partial \Delta_{l+2 \ldots m+2}^{n+1}-$ сферы в $Q$. Положим $K=Q \bigcup_{0=\widetilde{0}, m=\widetilde{m}} \widetilde{Q}$.

Незацепленность в лемме 6.1. а следует из того, что $\Sigma^{l}$ (соответственно $\widetilde{\Sigma}^{l}$ ) ограничивает диск $\Delta_{01 \ldots l+1}^{l+1}\left(\right.$ соответственно $\left.\widetilde{\Delta}_{01 \ldots l+1}^{l+1}\right)$ в $K-\widetilde{\Sigma}^{n}\left(\right.$ соответственно в $\left.K-\Sigma^{n}\right)$.

Чтобы доказать вторую часть леммы 6.1.а для $m>n+2$, достаточно показать, что $P$ не вложим в $\mathbb{R}^{m}$ (ср. [131; доказательство леммы 1.4$\left.]\right)$. Значит, достаточно доказать, что не существует эквивариантного отображения $\widetilde{P} \rightarrow S^{m-1}$. Это следует из [131; конструкция перед леммой 1.2, лемма 1.2], [161]. Для $m=n+2$ вторая часть 6.1.а следует из [131; лемма 1.4].

В первых двух абзацах доказательства леммы 1.1 из [131] фактически доказано, что $Q$ вложимо в $\mathbb{R}^{m}$. Так $m \geqslant n+2$, то сушествует и вложение $K \subset \mathbb{R}^{m}$. Если $m>n+2$, то свойство b) леммы 6.1 справедливо для любого вложения $K \subset \mathbb{R}^{m}$ [96; теорема 8]. Если $m=n+2$, то для нашего вложения $Q \subset \mathbb{R}^{m}$ сфера $\Sigma^{n}$ незаузлена в $\mathbb{R}^{m}$. Мы можем вложить в $\mathbb{R}^{m}$ две копииполиэдра $Q$ далеко друг от друга. Соединим две точки $\Sigma^{n}$ и $\widetilde{\Sigma}^{n}$ дугой и притянем эти точки сфер друг к другу вдоль этой дуги. Сделав аналогичные построения для $\Sigma^{l}$ и $\widetilde{\Sigma}^{l}$, мы получим требуемое вложение.

ЛЕмма 6.2 (ср. [131; 2$]$ ). Пусть $K$ - полиэдр и $K \subset \mathbb{R}^{m}$ - вложение из леммы 6.1. Пусть $D^{n} \subset \Sigma^{n}$ и $\widetilde{D}^{n} \subset \widetilde{\Sigma}^{n}-P L$ диски во внутренностях тех двух n-симплексов некоторой триангуличи полиәдра $K$, которые содержат единственную общую точку сфер $\Sigma^{n} u \widetilde{\Sigma}^{n}$. Тогда существует такое PL отображение $g: K \rightarrow \mathbb{R}^{m}$, что

a) $\left.g\right|_{K-\stackrel{\circ}{D}^{n}}-$ включение, $\left.g\right|_{K-\underset{D^{n}}{ }}-$ вложсение, но $g\left(D^{n}\right) \cap g\left(\widetilde{D}^{n}\right) \neq \varnothing$;

b) произведение Уайтхеда (произвольно ориентированных) отображсений включения $\Sigma^{l}$ и $\widetilde{\Sigma}^{l}$ в $\Sigma^{l} \vee \widetilde{\Sigma}^{l} \subset \mathbb{R}^{m}-g\left(\Sigma^{n} \vee \widetilde{\Sigma}^{n}\right)$ гомотопно нулю.

ДокАЗАТЕльство. Возьмем точки $a \in \stackrel{\circ}{D^{n}}, \widetilde{a} \in \widetilde{\stackrel{\circ}{D}^{n}}$ и дугу $s \subset \mathbb{R}^{m}$, соединяюшую $a$ с $\widetilde{a}$. По соображениям общего положения $s \cap K=\{a, \widetilde{a}\}$. Сделаем пальцевое движение диска $D^{n}$ вдоль дуги $s$ (т.е. построим новое вложение $D^{n} \rightarrow \mathbb{R}^{m}$, получаюшееся из старого выпусканием $n$-мерного пальца из $D^{n}$ вдоль дуги $s$, рис. 6.1). Получим новое PL отображение $g: K \rightarrow \mathbb{R}^{m}$, для которого справедливо свойство а) леммы 6.2. По соображениям общего положения $\operatorname{dim}\left(g\left(D^{n}\right) \cap \widetilde{D}^{n}\right) \leqslant 2 n-m$ и $g\left(D^{n}\right)$ пересекает $\widetilde{D}^{n}$ трансверсально. Мы можем представить регулярную окрестность $B^{m}$ произвольной точки $c$ этого пересечения как произведение $B^{2 n-m} \times B^{l+1} \times B^{l+1}$ шаров, где $B^{2 n-m} \times 0 \times 0$ отвечает пересечению, $B^{2 n-m} \times B^{l+1} \times 0$ и $B^{2 n-m} \times 0 \times B^{l+1}$ 
отвечают $g\left(D^{n}\right)$ и $\widetilde{D}^{n}$ соответственно (через 0 мы обозначаем центр $B^{k}$ ). В окрестности точки $c$ имеется тор $0 \times \partial B^{l+1} \times \partial B^{l+1}$ (назьваемьй характеристическим). По лемме 6.1.b $\mathbb{R}^{m}-g\left(\Sigma^{n} \vee \widetilde{\Sigma}^{n}\right) \simeq S^{l} \vee S^{l}$. Обозначим через $\alpha$ и $\widetilde{\alpha}$ элементы групшы $\pi_{l}\left(\mathbb{R}^{m}-g\left(\Sigma^{n} \vee \widetilde{\Sigma}^{n}\right)\right)$, представляемые гомеоморфизмами $S^{l} \rightarrow y \vee S^{l}$ и $S^{l} \rightarrow S^{l} \vee y$ $\left(y \in S^{l}\right)$ соответственно (с некоторыми ориентациями). С должными ориентациями включения $0 \times \partial B^{l+1} \times y$ и $0 \times y \times \partial B^{l+1}$ в $\mathbb{R}^{m}-g\left(\Sigma^{n} \vee \widetilde{\Sigma}^{n}\right)$ гомотопны сфероидам $\alpha$ и $\widetilde{\alpha}$ соответственно. Так как отображение

$$
[\alpha, \widetilde{\alpha}]: S^{2 l-1} \rightarrow S^{l} \vee S^{l} \cong\left(0 \times y \times \partial B^{l+1}\right) \vee\left(0 \times \partial B^{l+1} \times y\right)
$$

продолжается до отображения $B^{2 l} \rightarrow 0 \times \partial B^{l+1} \times \partial B^{l+1}$ [14], [86], [30], то [ $\left.\alpha, \widetilde{\alpha}\right]$ нуль-гомотопно в $\mathbb{R}^{m}-g\left(\Sigma^{n} \vee \widetilde{\Sigma}^{n}\right)$. Пусть $p=\operatorname{link}\left(\Sigma^{l}, \Sigma^{n}\right)$ и $\tilde{p}=\operatorname{link}\left(\widetilde{\Sigma}^{l}, \widetilde{\Sigma}^{n}\right)$. По лемме 6.1.а $p$ и $\tilde{p}$ ненулевые. Включения $\Sigma^{l}$ и $\widetilde{\Sigma}^{l}$ в $\mathbb{R}^{m}-g\left(\Sigma^{n} \vee \widetilde{\Sigma}^{n}\right)$ представляют элементы $p \alpha$ и $\tilde{p} \widetilde{\alpha}$ групшы $\pi_{l}\left(\mathbb{R}^{m}-g\left(\Sigma^{n} \vee \widetilde{\Sigma}^{n}\right)\right)$ соответственно. Поэтому утверждение 6.2.b верно, так как $[p \alpha, \tilde{p} \widetilde{\alpha}]=p \tilde{p}[\alpha, \widetilde{\alpha}]=0$ в $\mathbb{R}^{m}-g\left(\Sigma^{n} \vee \widetilde{\Sigma}^{n}\right)$.

ЛЕмма 6.3 (ср. [131; леммы 2.1, 2.2], [29; $3.2, \S 4])$. Пусть $g: K \rightarrow \mathbb{R}^{m}$ - отображение из леммы 6.2 и $r: B^{2 l} \rightarrow \mathbb{R}^{m}-g\left(\Sigma^{n} \vee \widetilde{\Sigma}^{n}\right)-$ такое $P L$ отображение, что $\left.r\right|_{\partial B^{2 l}}: \partial B^{2 l} \rightarrow \Sigma^{l} \vee \widetilde{\Sigma}^{l}$ представляет произведение Уайтхеда включений $\Sigma^{l} \subset \Sigma^{l} \vee \widetilde{\Sigma}^{l} u \widetilde{\Sigma}^{l} \subset \Sigma^{l} \vee \widetilde{\Sigma}^{l}$. Положим

$$
Y=\left(K-\stackrel{\circ}{D}^{n}\right) \cup r\left(B^{2 l}\right) \cup g\left(D^{n}\right) \subset \mathbb{R}^{m} \quad u \quad R=\left(K-\stackrel{\circ}{D}^{n}\right) \cup r\left(B^{2 l}\right) \underset{\partial B^{n}=\partial D^{n}}{\bigcup} B^{n} .
$$

Тогда $\operatorname{dim} R=n, R$ квази-гомеоморфен $Y$ (значит, $R$ квазивложим в $\left.\mathbb{R}^{m}\right)$, но топологически не вложим в $\mathbb{R}^{m}$.

ДокаЗАТЕльство. Так как $m \leqslant \frac{3 n}{2}+1$, то $2 l \leqslant n$ и $\operatorname{dim} Y=\operatorname{dim} R=n$. Имеем $R \supset\left(K-\stackrel{\circ}{D^{n}}\right) \cup B^{n} \cong K$. Отсюда и из построения шаров $D^{n}$ и $\widetilde{D}^{n}$ следует, что шары $B^{n}$ и $\widetilde{D}^{n}$ содержатся во внутренностях двух пересекаюшихся симплексов некоторой триангуляции полиэдра $R$. Итак, существует очевидное отображение $R \rightarrow Y$, сингулярное множество которого содержится в $B^{n} \cup \widetilde{D}^{n}$, а значит, во внутренностях двух пересекаюшихся $n$-симплексов некоторой триангулящии полиэдра $R$. Следовательно, аналогично [131; лемма 2.1], $R$ квази-гомеоморфен $Y$.

Предположим, что сушествует вложение $h: R \rightarrow S^{m}$. Используя для $m>n+2$ [12], а для $m=n+2$ - следуюшее ниже замечание, мы можем считать, что $h$ является PL вложением. Пусть $\Sigma_{1}^{n}=\left(\Sigma^{n}-\stackrel{\circ}{D}^{n}\right) \bigcup_{\partial B^{n}=\partial D^{n}} B^{n} \subset R$. Отображение $\left.h \circ r\right|_{\partial B^{2 l}}$ может быть продолжено до отображения $h \circ r: B^{2 l} \rightarrow S^{m}-h\left(\Sigma_{1}^{n} \vee \widetilde{\Sigma}^{n}\right)$. Стало быть, $\left.h \circ r\right|_{\partial B^{2 l}}$ гомотопически тривиально в $S^{m}-h\left(\Sigma_{1}^{n} \vee \widetilde{\Sigma}^{n}\right)$. Теперь мы докажем обратное и придем к противоречию.

В случае $m>n+2$, как и в доказательстве свойства $6.1 . \mathrm{b}$, имеем $S^{m}-h\left(\Sigma_{1}^{n} \vee \widetilde{\Sigma}^{n}\right) \simeq$ $S^{l} \vee S^{l}$. Пусть $q=\operatorname{link}\left(h \Sigma^{l}, h \Sigma_{1}^{n}\right) \neq 0$ и $\tilde{q}=\operatorname{link}\left(h \widetilde{\Sigma}^{l}, h \widetilde{\Sigma}^{n}\right) \neq 0$. Обозначим через $\beta$ и $\widetilde{\beta}$ элементы групшы $\pi_{l}\left(\mathbb{R}^{m}-h\left(\Sigma_{1}^{n} \vee \widetilde{\Sigma}^{n}\right)\right)$, представляемые гомеоморфизмами $S^{l} \rightarrow y \vee S^{l}$ и $S^{l} \rightarrow S^{l} \vee y\left(y \in S^{l}\right)$ соответственно (с выбранными ориентациями). Значит, гомотопический класс отображения $\left.h \circ r\right|_{\partial B^{2 l}}: \partial B^{2 l} \rightarrow S^{m}-h\left(\Sigma_{1}^{n} \vee \widetilde{\Sigma}^{n}\right)$ можно рассматривать как элемент $q \tilde{q}[\beta, \widetilde{\beta}]$ групшы $\pi_{2 l-1}\left(S^{m}-h\left(\Sigma_{1}^{n} \vee \widetilde{\Sigma}^{n}\right)\right)$. По теореме 
Хилтона [ 115 ; дополнения к лекциям 5 и 6, c. 231,257$]$ отображение $\varphi: \pi_{2 l-1}\left(S^{2 l-1}\right) \rightarrow$ $\pi_{2 l-1}\left(S^{l} \vee S^{l}\right)$, определенное формулой $\varphi(\gamma)=[\beta, \widetilde{\beta}]$ ○ $\gamma$, является инъекцией. Это можно доказать также, используя вместо теоремы Хилтона точную последовательность $[62 ; 5.3]$. Значит, $[\beta, \widetilde{\beta}]$ имеет бесконечный порядок. Из этого следует, что элемент $q \tilde{q}[\beta, \widetilde{\beta}]$ нетривиален.

В случае $m=n+2$, по свойству 6.1.а, как и в [29; лемма 7], применяя теорему Столлингса [143], мы получаем, что коммутатор отображений $\Sigma^{l} \subset \Sigma^{l} \vee \widetilde{\Sigma}^{l} \subset \mathbb{R}^{m}-$ $h\left(\Sigma^{n} \vee \widetilde{\Sigma}^{n}\right)$ и $\widetilde{\Sigma}^{l} \subset \Sigma^{l} \vee \widetilde{\Sigma}^{l} \subset \mathbb{R}^{m}-h\left(\Sigma^{n} \vee \widetilde{\Sigma}^{n}\right)$ ненулевой.

ЗАМЕЧАНИЕ ДЛЯ $m=n+2$ и ТОПОЛОГИЧЕСКИХ ВЛОЖЕНИЙ (cp. [131; доказательство леммы 2.2]). Сушествуют сколь угодно близкие PL аптроксимации $h^{\prime}: R \rightarrow \mathbb{R}^{m}$ вложения $h$ такие, что $h^{\prime} \sigma \cap h^{\prime} \tau=\varnothing$ для любых двух непересекающихся симплексов $\sigma, \tau$ некоторой триангуляции полиэдра $R$. По соображениям обшего положения мы можем считать, что $\left.h^{\prime}\right|_{\Sigma^{1}},\left.h^{\prime}\right|_{\widetilde{\Sigma}^{1}}$ - вложения. Значит, по [131; лемма 1.4]

$$
\operatorname{link}_{\bmod 2}\left(h^{\prime} \Sigma_{1}^{n}, h^{\prime} \Sigma^{1}\right)=\operatorname{link}_{\bmod 2}\left(h^{\prime} \widetilde{\Sigma}^{n}, h^{\prime} \widetilde{\Sigma}^{1}\right)=1 .
$$

Незаузленнсть пар $\Sigma^{n}, \widetilde{\Sigma}^{1}$ и $\widetilde{\Sigma}^{n}, \Sigma^{1}$ доказывается аналогично PL случаю. Остаток доказательства аналогичен PL случаю $\left(h \rightarrow h^{\prime}\right)$, поскольку там используется только свойство 6.1.а, а не тот факт, что $h^{\prime}$ - вложение.

\section{§7. Трюк Уитни}

В этом параграфемы докажем гладкую вложимость гладких $n$-многообразий в $\mathbb{R}^{2 n}$ (теорема 3.1) и критерий вложимости $n$-полиэдров в $\mathbb{R}^{2 n}$ (теорема 2.1.а) для $n \geqslant 3$. Доказательства этих теорем основаны на конструкциях, являюшихся многомерными обобщениями движений Райдемайстера (рис. 2.2). Наиболее важная из этих конструкций, отвечаюшая второму движению Райдемайстера (рис. 2.2.b), носит название трюка Уитни. Отметим, что эта конструкция используется не только в теории вложений, но также во многих других разделах геометрической топологии. Проиллюстрируем конструкцию трюка Уитни на следующем примере.

ИДЕЯ ДОКАЗАТЕЛЬСТВА ТЕОРЕМЫ 3.1 В DIFF КАТЕГОРИИ. ИСПОЛЬЗУЯ МНОГОмерньй аналог первого движения Райдемайстера (рис. 2.2.а), любое гладкое отображение общего положения $f: N \rightarrow \mathbb{R}^{2 n}$ можно изменить так, чтобы добавилась одна точка самопересечения с предписанньм знаком. Следовательно, существует такое отображение $f: N \rightarrow \mathbb{R}^{2 n}$ обшего положения, что его сингулярности состоят из четного числа изолированных двойных точек с алгебраической суммой нуль.

Поэтому, чтобы завершить доказательство теоремы 3.1 , уничтожим эти двойные точки парами. Этот прием и назьвается трюком Уитни. Занумеруем двойные точки $x_{1}, y_{1}, x_{2}, y_{2} \in N$ так, чтобы $f\left(x_{1}\right)=f\left(x_{2}\right)$ и $f\left(y_{1}\right)=f\left(y_{2}\right)$. Далее, соединим $x_{1}$ с $y_{1}$ и $x_{2}$ с $y_{2}$ дугами $l_{1}$ и $l_{2}$ так, чтобы эти двойные точки имели противоположные знаки (рис. 7.1). По соображениям общего положения $(n \geqslant 2)$ ограничения $\left.f\right|_{l_{1}}$ и $\left.f\right|_{l_{2}}$ являются вложениями и $l_{1}, l_{2}$ не содержат других двойных точек отображения $f$. Поскольку $2 n \geqslant 4$, мы можем вложить 2 -диск $D$ в $\mathbb{R}^{2 n}$ так, чтобы $\partial D=f\left(l_{1}\right) \cup f\left(l_{2}\right)$. По соображениям общего положения $(n \geqslant 3)$ имеем $D \cap f(N)=\partial D$. Теперь мы можем 


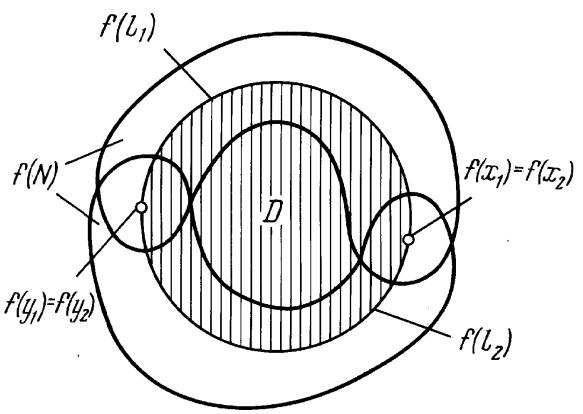

Рис. 7.1

сдвинуть $f$-образ регулярной окрестности дуги $l_{1}$ в $N$ вдоль $D$ так, чтобы "взаимно уничтожить" двойные точки $f\left(x_{1}\right)=f\left(x_{2}\right)$ и $f\left(y_{1}\right)=f\left(y_{2}\right)$ (детали см. в [84], [92]).

Введем обозначения, нужные для дальнейшего. Пусть $K-n$-полиэдр с триангуляцией $T$. Отображение $f: K \rightarrow \mathbb{R}^{m}$ является вложением тогда и только тогда, когда выполнены следующие условия:

$\left.f\right|_{\alpha}$ - вложение для каждого $\alpha \in T$;

$f \alpha \cap f \beta=\varnothing$ для всех $\alpha \times \beta \subset \widetilde{T}$;

$f \alpha \cap f \beta=f(\alpha \cap \beta)$ для всех $\alpha, \beta \in T$ таких, что $\alpha \cap \beta \neq \varnothing$.

ДОКАЗАТЕЛЬСТВО ТЕОРЕМЫ 2.1.а ДЛЯ $n \geqslant 3$. Возьмем отображение общего положения $\varphi: K \rightarrow \mathbb{R}^{m}$, линейное на симплексах некоторой триангуляции $T$ полиэдра $K$. Тогда условие (7.1.а) верно при замене $f$ на $\varphi$. По соображениям общего положения свойства (7.1.b) и (7.1.c) верны при замене $f$ на $\varphi$, за исключением случая $\operatorname{dim} \alpha=\operatorname{dim} \beta=n$. Доказательство теоремы 2.1.а состоит из трех частей: лемм 7.1, 7.2 и 7.3. Доказательство леммы 7.1 основано на пальиевых движениях Ван Кампена, обобщаюших пятое движение Райдемайстера (рис. 2.2.е). Лемма 7.2 доказьвается индукцией по парам $n$-симплексов из $\widetilde{T}$ с применением трюка Уитни (мы опускаем подробности, их можно найти в [29]). Доказательство леммы 7.3 основано на многомерном обобшении четвертого движения Райдемайстера (рис. 2.2.d).

ЛЕмма 7.1 (ср. [29; лемма 2]). Пусть $K$ - такой n-полиәдр с триангулячией $T$, что $v(K)=0$. Пусть, далее, $\varphi: K \rightarrow \mathbb{R}^{2 n}$ - такое отображсние, что свойство (7.1.а) выполняется при замене $f$ на $\varphi$. Тогда существует отображсние общего положсения $f: K \rightarrow \mathbb{R}^{2 n}$, для которого выполнено свойство (7.1.а) $и$

$$
f \alpha \cdot f \beta=0 \text { для всех } \alpha \times \beta \subset \widetilde{T}
$$

ДокАЗАТЕльство. Из условия $v(K)=0$ вытекает, что $v_{\varphi}$ - эквивариантная кограница. Следовательно, $v_{\varphi}$ есть сумма некоторых “элементарных” эквивариантных кограниц $\delta\left(\sigma^{n} \times \nu^{n-1}\right)$ с $\sigma^{n} \times \nu^{n-1} \subset \widetilde{T}$. Применяя пальцевые движения Ван Кампена, т.е. многомерные обобшения пятого движения Райдемайстера (рис. 2.2.е), получаем требуемое отображение $f$. 
ЛЕмма 7.2 [29; лемма 4]. Пусть $n \geqslant 3, K-n$-полиэдр с триангуляцией $T$. Пусть, далее, $\varphi: K \rightarrow \mathbb{R}^{2 n}$ - такое отображсние, что свойства (7.1.а) и (7.2) выполнены для $f \rightarrow \varphi$. Тогда существует отображсение общего положения $f: K \rightarrow \mathbb{R}^{2 n}$, для которого выполняются свойства (7.1.a) и (7.1.b).

ЛЕмма 7.3 (ср. [29; лемма 5]). Пусть $n \geqslant 3, K-n$-полиәдр с триангуляцией Tu $\varphi: K \rightarrow \mathbb{R}^{2 n}$ - такое отображение, что свойства (7.1.a) и (7.1.b) выполняются при замене $f$ на $\varphi$. Тогда существует вложение $f: K \rightarrow \mathbb{R}^{2 n}$.

ДокАЗАТЕльство. По индукции можем считать, что условие (7.1.с) верно при замене $f$ на $\varphi$ для всех пар $(\alpha, \beta)$, за исключением $\left(\sigma^{n}, \tau^{n}\right)$. Кроме того, мы можем считать, что $\varphi \sigma^{n} \cap \varphi \tau^{n}$ есть точка (назовем ее $p$ ). Пусть $v$ - точка в $\sigma^{n} \cap \tau^{n}$. Возьмем $\mathrm{PL}$ дуги $l_{1} \subset v \cup \stackrel{\circ}{\sigma}^{p}$ и $l_{2} \subset \stackrel{\circ}{\tau} q$, соединяющие $v$ с прообразами точки $p$ и не содержащие двойных точек отображения $\varphi$ внутри себя (рис. 7.2$)$. Тогда $\varphi\left(l_{1} \cup l_{2}\right)-$ окружность. Поскольку $n \geqslant 3$, она является границей некоторого PL вложенного 2-диска $D^{2} \subset \mathbb{R}^{2 n}$. По соображениям общего положения $(n+2<2 n)$ имеем $\stackrel{\circ}{D^{2}} \cap \varphi K=\varnothing$. Регулярная окрестность диска $D^{2}$ в $\mathbb{R}^{m}$ rel $\varphi v$ является PL $2 n$-шаром $D^{2 n}$. Его прообраз $\varphi^{-1} D^{2 n}$ является регулярной окрестностью дуги $l_{1} \cup l_{2}$ в $K$ rel $v$ игомеоморфен букету $D^{n} \vee D^{n}$ двух PL $n$-шаров с обшей точкой на их границах. Согласно [96] ограничение $\varphi: \partial D^{n} \vee \partial D^{n} \rightarrow \partial D^{2 n}$ незаузлено. Значит, оно может быть продолжено до вложения $h: D^{n} \vee D^{n} \rightarrow D^{2 n}$. Чтобы завершить доказательство леммы, положим $f$ равным $\varphi$ на $K-\left(D^{n} \vee D^{n}\right)$ и равньм $h$ на $D^{n} \vee D^{n}$.
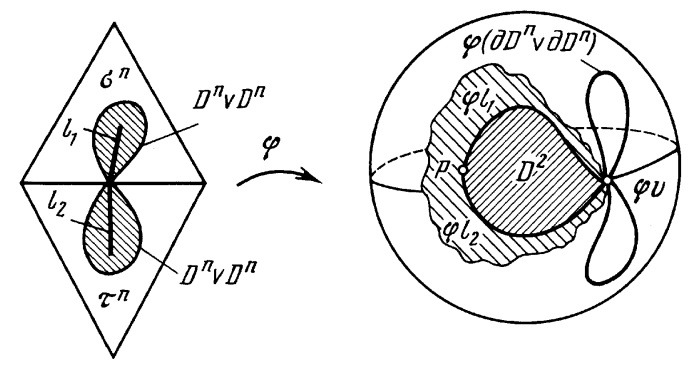

Рис. 7.2

Ван Кампен изобрел пальцевые движения для доказательства леммы 7.1. Нам понадобится их модификация, которую мы продемонстрируем на примере доказательства случая $m=2 n+1$ теоремы 4.1.е. Введем необходимые обозначения. Для отображений $E, G: D^{p} \times D^{q} \rightarrow S^{m-1}$ и гомотопии $h: \partial\left(D^{p} \times D^{q}\right) \times I \rightarrow S^{m-1}$ таких, что $h_{0}=E$ и $h_{1}=G$ на $\partial\left(D^{p} \times D^{q}\right)$, определим отображение $H_{E h G}: \partial\left(D^{p} \times D^{q} \times I\right) \rightarrow S^{m-1}$, полагая

$$
\left.H\right|_{D^{p} \times D^{q} \times 0}=E,\left.\quad H\right|_{D^{p} \times D^{q} \times 1}=G,\left.\quad H\right|_{\partial\left(D^{p} \times D^{q}\right) \times I}=h .
$$

Если $E=G$ на $\partial\left(D^{p} \times D^{q}\right)$, то $H_{E G}=H_{E i G}$, где $i$ обозначает постоянную гомотопию. Для любых отображений $f, f^{\prime}: D^{p} \sqcup D^{q} \rightarrow D^{m}$ таких, что $f D^{p} \cap f D^{q}=f^{\prime} D^{p} \cap$ $f^{\prime} D^{q}=\varnothing$ и $f^{\prime}=f$ на $D^{p} \sqcup \partial D^{q}$, определим отображение

$$
h_{f f^{\prime}}: S^{q} \cong D^{q} \underset{\partial D^{q}=\partial D_{+}^{q}}{\bigcup} D_{+}^{q} \rightarrow D^{m}-f D^{p},
$$


полагая

$$
h_{f f^{\prime}}(x)= \begin{cases}f(x), & x \in D^{q}, \\ f^{\prime}(x), & x \in D_{+}^{q} .\end{cases}
$$

Здесь $D_{+}^{q}$ - копия диска $D^{q}$. Если $f$ и $f^{\prime}$ - вложения и диск $f D^{p}$ незаузлен в $D^{m}$ (например, при $m-p \geqslant 3$ ), то $D^{m}-f D^{p} \simeq S^{m-p-1}$. Действительно, возьмем вложение $g: S^{m-p-1} \rightarrow D^{m}-f D^{p}$, для которого $g S^{m-p-1}$ трансверсально пересекает $f D^{p}$ ровно в одной точке. Тогда $g$ - гомотопическая эквивалентность, и индуцируемый ею изоморфизм гомотопических групп не зависит от $g$ (с точностью до умножения на \pm 1 ). Имеем $\left[h_{f f^{\prime}}\right] \in \pi_{q} S^{m-p-1}$. Так как $f^{\prime}=f$ на $D^{p} \sqcup \partial D^{q}$, то существует гомотопия $f_{t}: D^{p} \sqcup D^{q} \rightarrow D^{m}$ rel $D^{p} \sqcup \partial D^{q}$ между $f$ и $f^{\prime}$. Обозначая через $\tilde{f}, \widetilde{f}_{t}$ и $\tilde{f}^{\prime}$ сужения этих отображений на $D^{p} \times D^{q}, \partial\left(D^{p} \times D^{q}\right)$ и $D^{p} \times D^{q}$ соответственно, мы получаем в силу [153; лемма 1], что

$$
\left[H_{\widetilde{f} \widetilde{f}_{t} \tilde{f}^{\prime}}\right]=(-1)^{m-p_{\Sigma}}{ }^{p}\left[h_{f f^{\prime}}\right] \in \pi_{p+q} S^{m-1} .
$$

ДОКАЗАТЕЛЬСТВО СЛУЧАЯ $m=2 n+1$ ТЕОРЕМЫ 4.1.е. Приведем доказатеЛЬство для $n=1$ (общий случай доказывается аналогично). По соображениям общего положения любой граф $K$ вложим в $\mathbb{R}^{3}$, поэтому доказывать нужно только "реализуемость" любого отображения $\Phi: \widetilde{K} \rightarrow S^{m-1}$ вложением, т.е. сушествование такого вложения $f: K \rightarrow \mathbb{R}^{3}$, что $\tilde{f} \simeq_{\mathrm{eq}} \Phi$. Эта "реализуемость" уже не вытекает из соображений обшего положения. Пусть $T$ - триангуляция графа $K$. Докажем, что для каждой пары $(\sigma, \tau)$ ребер триангулящии $T$, подчиненных условию $\sigma \leqslant \tau$, существует такое вложение $f: K \rightarrow \mathbb{R}^{3}$, что $\widetilde{f} \simeq$ еq $\Phi$ на

$$
J=\bigcup\{\alpha \times \beta \cup \beta \times \alpha \subset \widetilde{T} \mid(\alpha, \beta)<(\sigma, \tau)\} .
$$

Теорема 4.1.е будет следовать из этого предложения, если положить $\sigma=\tau=$ (последний симплекс триангулящии $T$ ).

Доказательство сформулированного предложения ведется индукцией по $(\sigma, \tau)$. Если $\sigma$ и $\tau$ - первые ребра триангуляции $T$, то $\operatorname{dim} J=1$ и предложение следует из соображений общего положения. Предположим теперь по индукции, что $\left.\left.\widetilde{f}\right|_{J} \simeq_{\mathrm{eq}} \Phi\right|_{J}$ для вложения $f: K \rightarrow \mathbb{R}^{3}$. Если $\sigma \cap \tau \neq \varnothing$, то доказывать нечего, поэтому будем считать, что $\sigma \cap \tau=\varnothing$. Возьмем точки $a \in \stackrel{\circ}{\sigma}, b \in \stackrel{\circ}{\tau}$ и соединим их образы $f a$ и $f b$ дугой $l \subset \mathbb{R}^{3}$ такой, что $l \cap f K=\{f a, f b\}$. Пусть $D^{3}$ - достаточно малая регулярная окрестность дуги $l$ в $\mathbb{R}^{3}$. Тогда $f^{-1} D^{3}$ - несвязное объединение дуг $u \subset \stackrel{\circ}{\sigma}$ и $v \subset \stackrel{\circ}{\tau}$, которые являются регулярными окрестностями точек $a$ и $b$ в $K$. Можно считать, что дуги $f u$ и $f v$ незаузлены в $D^{3}$. Для проведения индукционного шага обернем несколько раз дугу $f v$ вокруг дуги $f u$ в $D^{3}$ (рис. 7.3).

В силу эквивариантной версии теоремы Борсука о продолжении гомотопии существует эквивариантное продолжение $\Psi: \widetilde{K} \rightarrow S^{2}$ отображения $\left.\widetilde{f}\right|_{J \cup(\sigma \times \tau-\stackrel{u}{ } \times \stackrel{\circ}{v})}$, для которого $\Psi \simeq_{\mathrm{eq}} \Phi$. Итак, $\Psi=\widetilde{f}$ на $\partial(u \times v)$.

Рассмотрим такое вложение $f^{+}: u \sqcup v \rightarrow D^{3}$, что $f^{+}=f$ на $u \sqcup \partial v$, и произвольную гомотопию $f_{t}$ rel $u \sqcup \partial v$ между $f$ и $f^{+}$. Из формулы (7.3) вытекает, что

$$
\left[H_{\Psi \tilde{f}_{t} \tilde{f}^{+}}\right]=\left[H_{\Psi \tilde{f}}\right]+\left[H_{\widetilde{f}_{\tilde{f}_{t}} \tilde{f}^{+}}\right]=\left[H_{\Psi \tilde{f}}\right]+\Sigma\left[h_{f f^{+}}\right] \in \pi_{2} S^{2}
$$




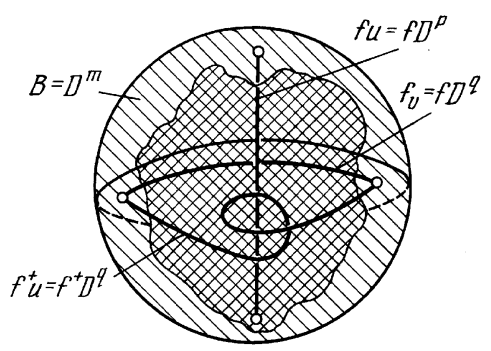

РиС. 7.3

(все отображения в этой формуле рассматриваются на $u \times v$ или на $\partial(u \times v)$ ). Для каждого элемента $\beta \in \pi_{1} S^{1}$ сушествует такое вложение $f^{+}: v \rightarrow D^{3}-f u$, что $\left[h_{f f^{+}}\right]=\beta$. Следовательно, по теореме Фройденталя о надстройке найдется отображение $f^{+}: v \rightarrow D^{3}-f u$, для которого $\left[H_{\Psi \tilde{f}_{t} f^{+}}\right]=0$. Продолжим отображение $f^{+}$на все $K$ посредством $f$. Получим такое отображение $f^{+}: K \rightarrow \mathbb{R}^{3}$, что $\tilde{f}^{+} \simeq_{\mathrm{eq}} \Psi \simeq_{\mathrm{eq}} \Phi$ на всем $J \cup \sigma \times \tau \cup \tau \times \sigma$. Этим завершается индукционный шаг и, следовательно, доказательство требуемого утверждения.

\section{§. Поглощение}

Идея поглощения впервые была придумана Зиманом и Столлингсом для доказательства гипотезы Пуанкаре при $n>4$ в кусочно-линейной и топологической категории [141], [164]. Эта идея стала одним из важнейших инструментов в геометрической топологии, особенно в теории вложений и изотопий. Поглощение из полиэдра $C$ можно интуитивно представлять себе, как вьпускание щупальцев из $C$, поглощающих $K$; размерность щупальца лишь на единищу больше размерности полиэдра $K$. В доказательстве теоремы 3.1 роль объединения таких щупальцев будет играть $c л е д$ полиэдра $K$ при сдавливании на $C$. Размерность новых пересечений полиэдра $K$ со щупальцами будет меньше исходной, и поэтому к новым пересечениям можно применить индукционньй процесс (см. ниже доказательство теоремы 3.1). Мы проиллюстрируем идею поглощения на примере доказательства теорем 3.1 и 3.2.a в PL категории. Дальнейшее обсуждение см. в [59].

ДоКАЗАТЕЛЬСТВо ТЕОРемЫ 3.1 в PL КАТЕГОРИИ. Так как каждое 2-многообразие является связной суммой торов и проективных плоскостей, то оно вложимо в $\mathbb{R}^{4}$. Пусть теперь $n \geqslant 3$. Без потери обшности можем считать, что $N$ связно. Рассмотрим PL отображение обшего положения $f: N \rightarrow \mathbb{R}^{2 n}$. Тогда $f$ имеет лишь конечное число двойных точек, т.е. таких точек $x, y \in N$, что $f(x)=f(y)$, но $x \neq y$. Обозначим эти точки через $x_{1}, y_{1}, \ldots, x_{p}, y_{p}$. Тогда $f\left(x_{i}\right)=f\left(y_{i}\right)$ и $f$ является вложением вне $\left\{x_{1}, y_{1}, \ldots, x_{p}, y_{p}\right\}$. Чтобы “убить" $x_{1}$ и $y_{1}$, возьмем дугу $l \subset N$, соединяющую $x_{1}$ с $y_{1}$ и не содержащую других точек $x_{i}, y_{i}$. Таким образом, $f(l)$ - простая замкнутая кривая в $\mathbb{R}^{2 n}$. Рассмотрим такой 2 -диск $D \subset \mathbb{R}^{2 n}$, что $\partial D=f(l)$ (рис. 2.2.а и 8.1). Так как $n+2<2 n$, то по соображениям общего положения имеем $D \cap f(N)=f(l)$. Возьмем регулярную окрестность $B$ диска $D$ в $\mathbb{R}^{2 n}$. Это $2 n$-шар. Если он достаточно мал, то $B_{0}=f^{-1}(B)$ - регулярная окрестность дуги $l$ в $N$. Значит, $B_{0}$ является $n$-шаром. Итак, мы поглотили $\Sigma(f)$ и $f \Sigma(f)$ в шары $B_{0}$ и $B$ соответственно. Так как 
каждый шар является конусом над своей гранищей, то вложение $f: \partial B_{0} \rightarrow \partial B$ может быть конически продолжено до вложения $f^{+}: B_{0} \rightarrow B$, равного $f$ на $\partial B_{0}$. Рассуждая аналогично для $i=1, \ldots, p$, мы “убьем" все двойные точки $x_{i}, y_{i}$ и получим вложение $N$ в $\mathbb{R}^{2 n}$.

Чтобы доказать теорему 3.2.а в PL категории, обобщим приведенное рассуждение. Для этого введем несколько важных понятий. Скажем, что полиэдр $Y$ получается из полиэдра $K$ әлементарным сдавливанием, если $K=Y \cup B^{n}$ и $Y \cap B^{n}=B^{n-1}$, где $B^{n-1}$ - грань шара $B^{n}$. Говорят, что это элементарное сдавливание осушествляется из $\mathrm{Cl}\left(\partial B^{n}-B^{n-1}\right)$ вдоль $B^{n}$ на $B^{n-1}$. Полиэдр $K$ сдавливается на $Y$ (обозначение: $K \searrow Y$ ), если найдется последовательность элементарных сдавливаний $K=K_{0} \searrow K_{1} \searrow K_{2} \searrow \cdots \searrow K_{n-1} \searrow K_{n}=Y$. Полиэдр $K$ сдавливаем, если он сдавливается на точку. Ясно, что шар $B^{n}$ сдавливаем, поскольку он сдавливается на свою грань $B^{n-1}$ и т. д. по индукции. Более того, конус $c K$ над компактным полиэдром $K$ сдавливаем (на свою вершину). Действительно, для каждого симплекса $A \subset K$ конус $c A$ сдавливается из $A$ на $c(\partial A)$, следовательно, $c K \searrow *$ индукцией по размерностям симплексов.

Сдавливание $K \searrow Y$ порождает деформационную ретракцию $r: K \rightarrow Y$, задаваемую деформациями каждого из шаров $B^{n}$ на его грань $B^{n-1}$. Рассмотрим гомотопию $H_{t}$ между тождественным отображением $K \rightarrow K$ и деформационной ретракцией $r: K \rightarrow Y$, индуцированной сдавливанием $K \searrow Y$. Следом подполиэдра $S$ в $K$ при сдавливании $K \searrow Y$ называется объединение полиэдров $H_{t}(S)$ для $t \in[0,1]$.

Пусть фиксировано вложение полиэдра $K$ в $\mathrm{PL}$ многообразие $M$. Окрестность $N$ полиэдра $K$ в $M$ называется регулярной, если $N$ - компактное замкнутое многообразие и $N \searrow K$. Один и тот же полиэдр может иметь разные регулярные окрестности. Но регулярная окрестность единственна с точностью до гомеоморфизма [154] и даже с точностью до изотопии, неподвижной на $K[75]$. Регулярная окрестность сдавливаемого полиэдра есть шар [154]. Обратное утверждение (т.е. что $K \subset B^{n}$ и $K \searrow *$ влечет $B \searrow K$ ) верно лишш в коразмерности $\geqslant 3$ [68]. Примем следуюшее соглашение. Символ $R_{M}(K)$, впервые появляющийся в данном контексте, будет обозначать произвольную регулярную окрестность полиэдра $K$ в $M$; в последующем $R_{M}(K)$ обозначает уже рассматривавшуюся регулярную окрестность.

ДОКАЗАТЕЛЬСТВО ТЕОРЕМЫ 3.2.а В PL КАТЕГОРИИ. Пусть $k=2 n-m-$ порядок связности многообразия $N$ и $f: N \rightarrow \mathbb{R}^{m}$ - отображение общего положения. Наша цель - "убить" сингулярное множество $\Sigma(f)$. Имеем $\operatorname{dim} \Sigma(f) \leqslant 2 n-m=k$. Основная часть доказательства заключается в построении таких сдавливаемых полиэдров $C \subset N$ и $D \subset \mathbb{R}^{m}$, что $S(f) \subset C$ и $f^{-1}(D)=C$. Полиэдры $C$ и $D$ являются аналогами дуги $l$ и диска $D$ из предыдушего рассуждения. Это построение основано на леммах 8.1, 8.2 о поглошении и приводится ниже. Считая эти полиэдры построенными, нетрудно завершить доказательство. Выберем такие регулярные окрестности $B^{n}=R_{N}(C)$ и $B^{m}=R_{\mathbb{R}^{m}}(D)$, что $f^{-1} B^{m}=B^{n}$. Поскольку $C$ и $D$ сдавливаемы, то $B^{m}$ и $B^{n}$ - шары. Определим новое отображение $g: N \rightarrow \mathbb{R}^{m}$ на $B^{n}$ как конус над $\left.f\right|_{\partial B^{n}}$, а вне $\stackrel{\circ}{B}^{n}$ как $g=f$. Так как $f$ - вложение вне $B^{n}$ и $B \cap f(N)=f\left(B^{n}\right)$, то $g-$ вложение. 
ЛЕмма О ПоГЛОшЕНИи 8.1 [141], [112]. Пусть данъ $(2 k+2-n)$-связное замкнутое $n$-многообразие $N$ и такой $k$-полиәдр $K \subset N$, что $n-k \geqslant 3$ и включение $K \subset N$ гомотопно нулю. Тогда $K$ поглощаем в $N$, т.е. содержится в некотором n-uаре $B \subset N$.

ОТНОСИТЕЛЬНАЯ ЛЕММА О ПОГЛОШЕНИИ 8.2. Пусть данъ $k$-связное замкнутое $P L$ п-многообразие $N, k$-полиэдр $K \subset N$ и сдавливаемый полиэдр $C \subset N$. Если $n-k \geqslant 3$, то $K$ поглощаем из $C$ в $N$, m.е. существует $R_{N}(C) \supset K$.

ПостроениЕ ПолиЭдРов $C$ и $D$. Так как $\operatorname{dim} \Sigma(f) \leqslant k=2 n-m, \operatorname{то} n-\operatorname{dim} \Sigma(f)$ $\geqslant n-k=m-n \geqslant 3$. Заметим также, что любое вложение $k$-полиэдра в $k$-связное многообразие гомотопно отображению в точку. Следовательно, по лемме о поглощении 8.1 существует $n$-шар $B \subset N$, содержаший $\Sigma(f)$. Для $\operatorname{dim} \Sigma(f)<\frac{n}{2}$ возьмем в этом шаре $B$ конус обшего положения $C$ над $\Sigma(f)$. В случае произвольного $\operatorname{dim} \Sigma(f)$ возьмем в качестве $C$ след полиэдра $\Sigma(f)$ при сдавливании шара $B$ в точку $x \notin \Sigma(f)$. Этот след назьвается сингулярным конусом над $\Sigma(f)$, а точка $x$ - его верииной (рис. 8.2). Аналогично, возьмем сингулярный конус $D \subset \mathbb{R}^{m}$ над $f(C)$. Пусть $S^{\prime}=f^{-1}(D)-C$.

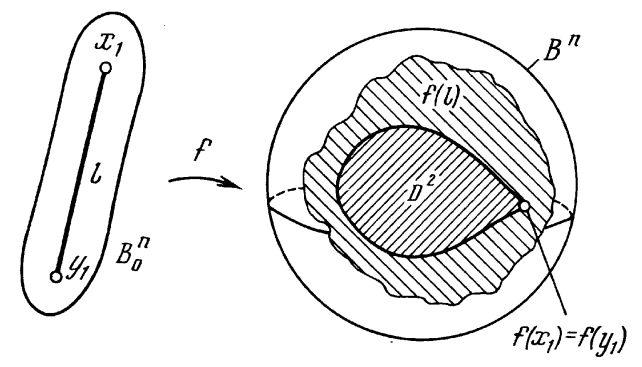

Рис. 8.1
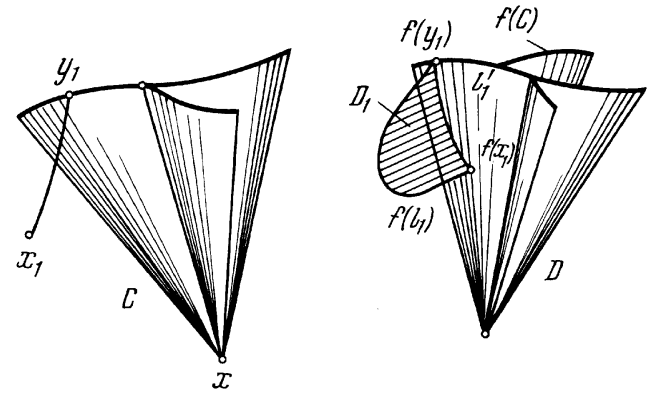

Рис. 8.2

В случае $m \geqslant \frac{3(n+1)}{2}$ имеем $(k+2)+n<m$, и по соображениям общего положения $D \cap f N=f C$, т.е. $S^{\prime}=\varnothing$. По определению сингулярные конусы $C$ и $D$ сдавливаемы. 
В случае $m=\frac{3 n}{2}+1$ множество $S^{\prime}$ может быть непустьм, но по соображениям общего положения его размерность не вьше $(k+2)+n-m=0$. Поэтому прежде чем "убить" $\Sigma(f)$, как в предыдущем рассуждении, нужно сперва "убить" множество $S^{\prime}$ размерности 0 . Пусть $S^{\prime}=\left\{x_{1}, \ldots, x_{p}\right\}$. Возьмем дугу $l_{1}$, соединяющую точку $x_{1}$ с любой точкой $y_{1}$ в $C$, и дугу $l_{1}^{\prime} \subset D$, соединяющую $f\left(x_{1}\right)$ с $f\left(y_{1}\right)$. Тогда $f\left(l_{1}\right) \cup l_{1}^{\prime}$ - окружность в $\mathbb{R}^{m}$. Выберем 2 -диск $D_{1}$, ограничиваемьй этой окружностью (рис. 8.2). Так как $2+n<m$, по соображениям общего положения имеем $D_{1} \cap f(N)=\partial D_{1}$. Конструируя такие попарно непересекаюшиеся дуги $l_{i} \subset N$ и диски $D_{i} \subset \mathbb{R}^{m}$ для каждой точки $x_{i}$, получим, что $f^{-1}(\widetilde{D})=\widetilde{C}$, где $\widetilde{C}=C \cup l_{1} \cup \cdots \cup l_{p}$ и $\widetilde{D}=D \cup D_{1} \cup \cdots \cup D_{p}$. Покажем, что $\widetilde{C}$ и $\widetilde{D}$ сдавливаемы. В самом деле, сдавливание $\widetilde{C}$ в точку получается сдавливанием каждой $l_{i} \searrow y_{i}$ и затем сингулярного конуса $C$ в его вершину. Аналогично $\widetilde{D} \searrow *$. Требуемые сдавливаемые полиэдры $\widetilde{C}$ и $\widetilde{D}$ построены.

Описанньй вьше переход от $m \geqslant \frac{3(n+1)}{2}$ к $m \geqslant \frac{3 n}{2}+1$ есть первьй шаг индукции, которая позволяет построить полиэдры $C$ и $D$ для $m \geqslant n+3$. На $j$-м шаге имеем $m \geqslant \frac{3 n+3-j}{2}$, следовательно, $\operatorname{dim} S^{\prime} \leqslant j-1$. Чтобы “убить” полиэдр $S^{\prime}$, достаточно найти “пленку" $C^{\prime}$ размерности не вьше $j$, “соединяющую” $S^{\prime}$ с $C$. По относительной лемме о поглощении 8.2 существует регулярная окрестность $B=R_{N}(C) \supset S^{\prime}$. Пусть $C^{\prime}$ - след полиэдра $S^{\prime}$ при сдавливании $B \searrow C$. Тогда $C^{\prime}$ сдавливаем на $C^{\prime} \cap C$ (здесь $C^{\prime}$ играет роль $l_{1} \cup \cdots \cup l_{n}$ в первом шаге). Выберем аналогичную “пленку" $D^{\prime}$ размерности не выше $j+1$, соединяюшую $f\left(C^{\prime}\right)$ с $D$ (рис. 8.2). Тогда $D^{\prime} \searrow D^{\prime} \cap D$. Однако $D^{\prime}$ может пересекаться с $f(N)$ не только по $f\left(C^{\prime}\right)$. Пусть $S^{\prime \prime}=f^{-1}\left(D^{\prime}\right)-C^{\prime}$. Тогда $\operatorname{dim} S^{\prime \prime} \leqslant j+1+n-m \leqslant j-2$, поскольку $m-n \geqslant 3$. Заметим, что мы добились уменьшения “сингулярной размерности”: $\operatorname{dim} S^{\prime \prime} \leqslant \operatorname{dim} S^{\prime}-1$. Следовательно, аналогично предыдущему шагу индукции, мы можем “убить" $S^{\prime \prime}$, т.е. построить полиэдр $C^{\prime \prime}$, содержащий $S^{\prime \prime}$ и сдавливаемьй на $C^{\prime \prime} \cap\left(C^{\prime} \cup C\right)$, и полиэдр $D^{\prime \prime}$, сдавливаемый на $D^{\prime \prime} \cap\left(D^{\prime} \cup D\right)$, так что $f^{-1}\left(D^{\prime \prime}\right)=C^{\prime \prime}$. Тогда полиэдры $\widehat{C}=C \cup C^{\prime} \cup C^{\prime \prime}$ и $\widehat{D}=D \cup D^{\prime} \cup D^{\prime \prime}$ сдавливаемы и $f^{-1}(\widetilde{D})=\widetilde{C}$. Таким образом, мы “убиваем" $S^{\prime}$ и завершаем $j$-й шаг индукции. Эти рассуждения проходят для всех $j \leqslant k=2 n-m$, поскольку $\frac{3 n+3-k}{2}=n+3$.

\section{§. Первая часть доказательства теоремы 4.1.e}

Доказательство теоремы 4.1.е в PL случае состоит из двух шагов: теоремы 9.1 (аналога лемм 7.1 и 7.2) и теоремы 10.1 (аналога леммы 7.3). Теорема 9.1 доказывается по индукции. Она следует из предложения 9.2 ниже для $\sigma^{p}=\sigma^{q}=$ (последний симплекс $T)$.

ТЕОРема 9.1 [156]. Пусть $K-n$-полиәдр с триангуляцией $T, m \geqslant \frac{3(n+1)}{2} u$ $\Phi: \widetilde{K} \rightarrow S^{m-1}$ әквивариантное отображсние. Тогда существует такое $P L$ отображсене $f: K \rightarrow \mathbb{R}^{m}$, что свойства (7.1.a) $u$ (7.1.b) выполнены $u$

$$
\left.\left.\widetilde{f}\right|_{\widetilde{T}} \simeq \mathrm{eq} \Phi\right|_{\widetilde{T}}
$$


ПРЕДЛОЖЕНИЕ 9.2. При предположсениях теоремы 9.1 для каждых $\sigma^{p} \times \sigma^{q} \in$ $\widetilde{T}$ таких, что $\sigma^{p} \leqslant \sigma^{q}$, существует $P L$ отображсение $f: K \rightarrow \mathbb{R}^{m}$ такое, что верно свойство (7.1.a), свойство (7.1.b) верно для $(\alpha, \beta)<\left(\sigma^{p}, \sigma^{q}\right)$ и

$$
\tilde{f} \simeq_{\mathrm{eq}} \Phi н а J_{\sigma^{p} \sigma^{q}}=\cup\left\{\alpha \times \beta \cup \beta \times \alpha \subset \widetilde{T} \mid(\alpha, \beta)<\left(\sigma^{p}, \sigma^{q}\right)\right\}
$$

ПЕРВАЯ ЛЕММА О ШАРЕ 9.3. При условия предложения 9.2 существуют $P L$ uары $D^{m} \subset \mathbb{R}^{m}, D^{p} \subset \stackrel{\circ}{\sigma}^{p}, D^{q} \subset \stackrel{\circ}{\sigma}^{q}$ maкuе, что

a) $\left.f\right|_{D^{p}}$ u $\left.f\right|_{D^{q}}$ собственные вложения в $D^{m}$;

b) $f \sigma^{p} \cap f \sigma^{q} \subset \stackrel{\circ}{D^{m}}$;

c) $D^{p}=\sigma^{p} \cap f^{-1} D^{m} u D^{q}=\sigma^{q} \cap f^{-1} D^{m}$;

d) $D^{m} \cap f P=\varnothing$, где $P=\bigcup\left\{\alpha \in T \mid \alpha \cap \sigma^{q}=\varnothing u \alpha<\sigma^{p}\right\}$.

ДокАЗАТЕЛЬСтво. Из свойства (7.1.b) для $(\alpha, \beta)<\left(\sigma^{p}, \sigma^{q}\right)$ получаем, что $f \sigma^{p} \cap f \partial \sigma^{q}=f \partial \sigma^{p} \cap f \sigma^{q}=\varnothing$. По соображениям обшего положения $\operatorname{dim}\left(f \sigma^{p} \cap f \sigma^{q}\right) \leqslant$ $p+q-m$. Пусть $C_{1} \subset \sigma^{p}-$ след полиэдра $\sigma^{p} \cap f^{-1} \sigma^{q}$ при сдавливании $\sigma^{p} \searrow$ (точка в $\stackrel{\circ}{\sigma}^{p}$ ). Аналогично определим $C_{2} \subset \stackrel{\circ}{\sigma} q$. Полиэдры $C_{1}, C_{2}$ являются обобщениями дуг $l_{1}, l_{2}$ из трюка Уитни. Они сдавливаемы, $f\left(\sigma^{p} \cap \sigma^{q}\right) \subset f C_{1} \cap f C_{2}$ и $\operatorname{dim} C_{1}, \operatorname{dim} C_{2} \leqslant p+q-m+1$. Из свойства (7.1.b) для $(\alpha, \beta)<\left(\sigma^{p}, \sigma^{q}\right)$ имеем: $C_{1} \cap P=\varnothing$. По соображениям общего положения $\operatorname{dim}\left(f P \cap f \sigma^{q}\right) \leqslant n+q-m$, следовательно, $\operatorname{dim}\left(f P \cap f \sigma^{q}\right)+\operatorname{dim} C_{2}<q$ и $C_{2} \cap P=\varnothing$.

Рассмотрим последовательность сдавливаний из некоторого PL $m$-шара $J^{m}$ в $\mathbb{R}^{m}$, содержащего $f\left(\sigma^{p} \cap \sigma^{q}\right)$ внутри себя, в точку в $\stackrel{\circ}{J}$. Пусть $C-$ след полиэдра $C_{1} \cup C_{2}$ при таком сдавливании. Полиэдр $C$ является обобшением диска $D$ из трюка Уитни. Он сдавливаем, $C_{1} \cup C_{2} \subset C$ и $\operatorname{dim} C \leqslant p+q-m+2$. По соображениям общего положения $C \cap f \sigma^{p}=C_{1}, C \cap f \sigma^{q}=C_{2}$ и $C \cap P=\varnothing$. Здесь мы использовали неравенство $m \geqslant \frac{3(n+1)}{2}$. Как и в трюке Уитни, теперь легко проверить, что регулярные окрестности полиэдров $C, C_{1}, C_{2}$ в достаточно маленьких (согласованных) триангуляциях пространств $\mathbb{R}^{m}, \sigma^{p}, \sigma^{q}$ соответственно и есть требуемые шары.

ДоКАЗАТЕЛЬСТВо ПРЕДЛОЖЕНИЯ 9.2. Возьмем отображение обшего положения $f: K \rightarrow \mathbb{R}^{m}$, линейное на симплексах триангуляции $T$. Это отображение $f$ уже удовлетворяет свойству (7.1.a). По предположению индукции (на $\left.\left(\sigma^{p}, \sigma^{q}\right)\right)$ можем считать, что верны свойства (7.1.а) и (9.2), а свойство (7.1.b) верно для $(\alpha, \beta)<\left(\sigma^{p}, \sigma^{q}\right)$. Мы можем также считать, что $f$ находится в общем положении. Предположим, что $p+q \geqslant m-1$ (иначе индукционньй шаг можно получить просто по обшему положению).

Первая часть доказательства - обобшение трюка Уитни - получение свойства (7.1.b) для $(\alpha, \beta)=\left(\sigma^{p}, \sigma^{q}\right)$. Возьмем PL шары $D^{m}, D^{p}, D^{q}$ из первой леммы о шаре. Из свойства (7.1.b) и свойства а) в лемме 9.3 следует, что $f \partial D^{p} \cap f D^{q}=f \partial D^{q} \cap$ $f D^{p}=\varnothing$. Так как $m-p \geqslant 3$, то $D^{m}-f D^{p} \simeq S^{m-p-1}$ (см. замечание перед (7.3)). Гомотопический класс в $\pi_{q-1} S^{m-p-1}$ отображения $\left.f\right|_{\partial D^{q}}: \partial D^{q} \rightarrow D^{m}-f D^{p}$ назьвается коэффичиентом пересечения $\left.f\right|_{\partial D^{p}}$ и $\left.f\right|_{\partial D^{q}}$ иобозначается $I\left(\left.f\right|_{D^{q}},\left.f\right|_{D^{q}}\right)$. Имеем $\Sigma^{p} I\left(\left.f\right|_{D^{p}},\left.f\right|_{D^{q}}\right)=(-1)^{m-p}\left[\left.\widetilde{f}\right|_{\partial\left(D^{p} \times D^{q}\right)}\right]=\left[\left.\Phi\right|_{\partial\left(D^{p} \times D^{q}\right)}\right]=0$. Здесь первое равенство верно по [82], [153; утверждение 1]. Второе равенство верно, так как $\tilde{f} \simeq \Phi$ 
на $\partial\left(\sigma^{p} \times \sigma^{q}\right)$ по индукционному предположению. Третье равенство верно, так как $\Phi$ определено на $\widetilde{T} \supset D^{p} \times D^{q}$. Так как $q \leqslant 2(m-p-1)-1$, из теоремы Фрейденталя о надстройке получаем, что вложение $\left.f\right|_{\partial D^{q}}$ продолжается до отображсения $f^{\prime}: D^{q} \rightarrow D^{m}-f D^{p}$. Здесь мы использовали неравенство $2 p+q \leqslant 2 m-3$.

Так как $2 q-m+1 \leqslant m-p-2$, из теоремы 3.2.а (на самом деле, нам нужен лишь случай $m \geqslant \frac{3(n+1)}{2}$ этой теоремы) следует, что $f^{\prime}$ гомотопно $\left(\operatorname{rel} \partial D^{q}\right)$ вложскию $f^{+}: D^{q} \rightarrow D^{m}-f D^{p}$. Здесь мы вновь использовали неравенство $p+2 q \leqslant 2 m-3$. Так как $m-q \geqslant 3$, то из относительной версии теоремы 3.5 [166; следствие 1 теоремы 9] следует, что сушествует объемлемая изотопия $h_{t}: D^{m} \rightarrow D^{m} \operatorname{rel} \partial D^{m}$ между $\left.f\right|_{D^{q}}$ и $f^{+}$. Продолжим $f^{+}$на $K$ по формуле

$$
f^{+}(x)= \begin{cases}h_{1}(f(x)), & \text { если } f(x) \in D^{m} \text { и } x \in \gamma \text { для некоторого } \gamma \supset \sigma^{p}, \\ f(x) & \text { иначе. }\end{cases}
$$

Легко проверить, что $f^{+}$удовлетворяет свойствам (7.1.b) для $(\alpha, \beta) \leqslant\left(\sigma^{p}, \sigma^{q}\right),(9.2)$ и (7.1.a).

Вторая часть доказательства - обобшение пальцевых движений Ван Кампена - получение свойства $(9.2)$ для $(\alpha, \beta)=\left(\sigma^{p}, \sigma^{q}\right)$, предполагая верньми свойства (7.1.а), (7.1.b) для $(\alpha, \beta) \leqslant\left(\sigma^{p}, \sigma^{q}\right)$ и свойство $(9.2)$ для $(\alpha, \beta)<\left(\sigma^{p}, \sigma^{q}\right)$. Мы начнем с аналога первой леммы о шаре. По соображениям общего положения мы можем взять такие точки $a \in \stackrel{\circ}{\sigma}^{p}-\Sigma(f)$ и $b \in \stackrel{\circ}{\sigma}^{q}-\Sigma(f)$, что ограничения отображения $f$ на некоторые малые окрестности точек $a$ и $b$ - вложения. Так как $x, y \leqslant m-2$, то мы можем соединить точки $f a$ и $f b$ такой дугой $l \subset \mathbb{R}^{m}$, что $l \cap f K=\{f a, f b\}$. Пусть $D^{m}=R_{\mathbb{R}^{m}}(l)$. Тогда $f^{-1} D^{m}$ - несвязное объединение $\mathrm{PL}$ дисков $D^{p} \subset \stackrel{\circ}{\sigma} p$ и $D^{q} \subset \stackrel{\circ}{\sigma}$, являющихся регулярными окрестностями в $K$ точек $a$ и $b$ соответственно.

По теореме Борсука о продолжении гомотопии сушествует такое продолжение $\Psi: \widetilde{T} \rightarrow S^{m-1}$ отображения $\left.\widetilde{f}\right|_{J \cup\left(\sigma^{p} \times \sigma^{q}-\stackrel{\circ}{D}^{p} \times \stackrel{\circ}{D}^{q}\right)}$, что $\Psi \simeq \Phi$. Итак, $\Psi=\widetilde{f}$ на $\partial\left(D^{p} \times D^{q}\right)$. Мы можем считать, что $\Psi=\Phi$. По (7.3) для каждого отображения $f^{\prime}: D^{p} \sqcup D^{q} \rightarrow D^{m}$ такого, что $f^{\prime}=f$ на $D^{p} \sqcup \partial D^{q}$ и $f^{\prime} D^{p} \cap f^{\prime} D^{q}=\varnothing$, и гомотопии $f_{t} \operatorname{rel} D^{p} \sqcup \partial D^{q}$ между $f$ и $f^{\prime}$ имеем:

$$
\left[H_{\Phi \tilde{f}_{t} \tilde{f}^{\prime}}\right]=\left[H_{\Phi \tilde{f}}\right]+\left[H_{\tilde{f} \tilde{f}_{t} \tilde{f}^{\prime}}\right]=\left[H_{\Phi \tilde{f}}\right]+(-1)^{m-p} \Sigma^{p}\left[h_{f f^{\prime}}\right] \in \pi_{p+q} S^{m-1}
$$

(здесь через $\Phi, \tilde{f}, \tilde{f}^{+}$и $\widetilde{f}_{t}$ обозначены сужения этих отображений на $D^{p} \times D^{q}$ и $\partial\left(D^{p} \times D^{q}\right)$ соответственно). Так как $2 p+q \leqslant 2 m-3$, то $q \leqslant 2(m-p-1)-1$, и из теоремы Фрейденталя о надстройке получаем, что $\Sigma^{p}-$ эпиморфизм. Так как для каждого элемента $\beta \in \pi_{q} S^{m-p-1}$ существует отображение (не обязательно вложение) $f^{\prime}: D^{q} \rightarrow D^{m}-f D^{p}$ такое, что $\left[h_{f f^{\prime}}\right]=\beta$ и $f^{\prime}=f$ на $D^{p} \sqcup \partial D^{q}$, то мы можем взять $f^{\prime}$ таким, что $\left[H_{\Phi} \tilde{f}_{t} \tilde{f}^{\prime}\right]=0$. Здесь мы вновь использовали неравенство $p+2 q \leqslant 2 m-3$.

Завершение доказательства такое же, как в обобшении трюка Уитни.

\section{$\S$ 10. Вторая часть доказательства теоремы 4.1.e}

Tеорема 10.1 ([136], ср. [153]). Пусть $K-n$-полиәдр с триангуляцией $T, m \geqslant$ $\frac{3(n+1)}{2} u \varphi: K \rightarrow \mathbb{R}^{m}-P L$ отображсение такое, что выполнены свойства (7.1.а), (7.1.b) $u$ 
(А) для любого $\alpha \in T$ существуют гомеоморфизмь

$$
R(\alpha, \partial \alpha) \cong \operatorname{lk} \alpha * \alpha \quad u \quad R_{\mathbb{R}^{m}}(f \alpha, f \partial \alpha) \cong \mathrm{lk} f \alpha * f \alpha
$$

(для некоторой триангуляции $\mathbb{R}^{m}$, не зависящей от $\alpha$ ) такие, что $f \mathrm{lk} \alpha \subset \mathrm{lk} f \alpha$ $\left.u f\right|_{R(\alpha, \partial \alpha)}=\left.\left.f\right|_{\mathrm{lk} \alpha} * f\right|_{\alpha}$.

Тогда существует $P L$ вложсние $f: K \rightarrow \mathbb{R}^{m}$ такое, что

$$
\left.\widetilde{f}\right|_{\widetilde{T}} \simeq \text { eq }\left.\varphi\right|_{\widetilde{T}}
$$

Здесь через lk обозначается линк симплекса в триангуляции [125]. Вторая (более сложная) часть доказательства в [153] содержит ошибку [153; с. 24, строки 9 и 18], которая по-видимому является чисто технической и может быть устранена при помоши тех же идей. Доказательство в [136] тоже содержит ошибку (явно не сформулировано используемое свойство (А) теоремы 10.1), которая исправлена здесь. Заметим, что свойство (А) (или его следствие - свойство $\left.f^{-1} R_{\mathbb{R}^{m}}(f \eta, f \partial \eta) \neq R_{K}(\eta, \partial \eta)\right)$ неверно для произвольного PL отображения обшего положения. Зато свойство (А) вьполнено для отображения, линейного на симплексах, а также сохраняется в процессе доказательства теоремы 9.1. Поэтому теорема 4.1.є действительно следует из теоремы 9.1 (точнее, ее версии с добавлением условия (А) в предположение и в заключение теоремы 9.1) и теоремы 10.1. Приводимое здесь доказательство теоремы 10.1 является обобщением доказательства леммы 7.3. Теорема 10.1 следует из предложения 10.2 ниже для $\sigma^{p}=\sigma^{q}=($ последний симплекс триангулящии $T)$.

ПРЕДЛОЖЕНИЕ 10.2. При предположсениях теоремы 10.1 для любъх $\sigma^{p} \times \sigma^{q} \in$ $T \times T$ таких, что $\sigma^{p} \leqslant \sigma^{q}$, существует $P L$ отображсение $f: K \rightarrow \mathbb{R}^{m}$ такое, что виполняются свойства (7.1.с) для $(\alpha, \beta)<\left(\sigma^{p}, \sigma^{q}\right)$, (7.1.a), (7.1.b) u (10.1).

ВТОРАЯ ЛЕММА О ШАРЕ 10.3. Для отображсения $f$, удовлетворяющего заключению предложсения 10.2, существуют $P L$ шарь $D^{r}=f\left(\sigma^{p} \cap \sigma^{q}\right), D^{p}, D^{q}, D^{m} \subset$ $\mathbb{R}^{m}$ такие, что

1) $D^{p} \subset D^{r} \cup f^{\circ} \stackrel{\circ}{p}^{p}$ u $D^{q} \subset D^{r} \cup f^{\circ} \sigma^{q}$;

2) $D^{p}=D^{m} \cap f \sigma^{p}$ u $D^{q}=D^{m} \cap f \sigma^{q}$ собственно вложены в $D^{m}$;

3) $D^{r}=\partial D^{p} \cap \partial D^{q}$;

4) $D^{r}$ незаузлен в $\partial D^{p}$ и в $\partial D^{q}$;

5) $\Sigma=\mathrm{Cl}\left(\left(f \sigma^{p} \cap f \sigma^{q}\right)-D^{r}\right) \subset \stackrel{\circ}{D^{m}} \cup D^{r}$;

6) $D^{m} \cap X \subset D^{r}$, где $X=\bigcup f\left\{\alpha \in T \mid \alpha \cap \sigma^{p}=\varnothing\right.$ или $\left.\alpha<\sigma^{q}\right\}$.

ДОКАЗАТЕЛЬСТВО ПРЕДЛОЖЕНИЯ 10.2 С ИСПОЛЬЗОВАНИЕМ ВТОРОЙ ЛЕММЫ О ШАРЕ. Отображение $\varphi$ уже удовлетворяет свойствам (7.1.a), (7.1.b) и (10.1). Мы достигаем свойства (7.1.с) для $(\alpha, \beta)<\left(\sigma^{p}, \sigma^{q}\right)$ индукцией по $\left(\sigma^{p}, \sigma^{q}\right)$. База $\sigma^{p}=$ (первьй симплекс $T$ ) получается при $f=\varphi$. Предположим теперь, что $f$ удовлетворяет свойствам (7.1.c) для $(\alpha, \beta)<\left(\sigma^{p}, \sigma^{q}\right)$, (7.1.a), (7.1.b) и (10.1). Можно считать, что $f$ находится в общем положении. Предположим, что $p+q \geqslant m$ и $\sigma^{p} \not \subset \sigma^{q}$ (в противном случае шаг индукции следует по соображениям общего положения или по предположению индукции). 
Возьмем шары $D^{r}, D^{p}, D^{q}, D^{m}$, данные второй леммой о шаре. Воспользуемся [96; теорема 9 и обсуждение перед ее формулировкой]: если $m-3 \geqslant p, q, S^{p}, S^{q} \subset S^{m}$ и $S^{p} \cap S^{q}=D^{r}$, где $D^{r}$ незаузлен в $S^{p}$ и в $S^{q}$, тогда $S^{p} \cup S^{q}$ незаузлено в $S^{m}$. Значит, мы можем считать, что вложение $\partial D^{p} \bigcup_{D^{r}} \partial D^{q} \subset \partial D^{m}$ стандартное. По относительной версии теоремы 3.5 [166; следствие 1 теоремы 9] можно считать, что вложение $\left(D^{p}, \partial D^{p}\right) \subset\left(D^{m}, \partial D^{m}\right)$ стандартно. Значит, вложение $\partial D^{q} \subset \partial D^{m}$ продолжается до нового вложения $D^{q} \rightarrow\left(\stackrel{\circ}{D^{m}}-D^{p}\right) \cup \partial D^{q}$. По относительной версии теоремы 3.5 это новое вложение объемлемо изотопно вложению $D^{q} \subset D^{m}$ rel $\partial D^{m}$. Поэтому сушествует изотопия $h_{t}: D^{m} \rightarrow D^{m} \mathrm{rel} \partial D^{m}$ такая, что $D^{p} \cap h_{1} D^{q}=D^{r}$. Определим отображение $f^{+}: K \rightarrow \mathbb{R}^{m}$ той же формулой, что и в доказательстве предложения 9.2. Очевидно, $f^{+}$удовлетворяет условиям 1$)-3$ ) второй леммы о шаре 10.3 . По (7.1.а) $\sigma^{p}$ и $\sigma^{q}$ не содержатся в границе одного и того же симплекса $T$. Из этого, $D^{p} \cap h_{1} D^{q}=D^{r}$ и свойств 5), 6) второй леммы о шаре 10.3 следует, что $f^{+}$удовлетворяет также условию (7.1.c) для $(\alpha, \beta) \leqslant\left(\sigma^{p}, \sigma^{q}\right)$. Шаг индукции обоснован.

ЛЕмМА О СДАВЛИВАНИи 10.4. (Следует из [20; теорема 3.1 и добавление 3.4$]$ ). Если $A$ и $B$ - регулярные окрестности полиэдра $Z$ в $P L$ многообразии $M \operatorname{rel} Y$ $u A \subset B, \operatorname{mo} B \searrow A \operatorname{rel} Y$.

ДОКАЗАТЕЛЬСТВо ВТОРОЙ ЛЕММЫ О ШАРЕ. По свойству (7.1.a) $D^{r}$ является PL шаром. Сделаем предварительные построения (ср. [153; $\S 6 \mathrm{a}])$. Пусть $S$ - линк некоторого $r$-симплекса из $\stackrel{\circ}{D^{r}}$ в некоторой триангуляции пространства $\mathbb{R}^{m}$. Тогда $S$ является PL $(m-r-1)$-сферой и $R_{\mathbb{R}}\left(D^{r}, \partial D^{r}\right) \cong S * D^{r}$. По свойству (А) из теоремы 10.1 $R_{\mathbb{R}^{m}}\left(D^{r}, \partial D^{r}\right) \cap f \alpha=R_{f \alpha}\left(D^{r}, \partial D^{r}\right)$ переходит в $(S \cap f \alpha) * D^{r}$ при этом гомоморфизме для любого $\alpha \in T$ (для $\alpha \not \supset \sigma^{p} \cap \sigma^{q}$ каждое из множеств $R_{\mathbb{R}^{m}}\left(D^{r}, \partial D^{r}\right) \cap f \alpha$, $R_{f \alpha}\left(D^{r}, \partial D^{r}\right)$ и $(S \cap f \alpha) * D^{r}$ пусто). Также $S \cap f \alpha$ является PL $(\operatorname{dim} \alpha-r-1)$-шаром для любого $\alpha \in T, \alpha \supset \sigma^{p} \cap \sigma^{q}$.

Возьмем различные точки $a \in\left(S \cap f \stackrel{\circ}{\sigma}^{p}\right)-X$ и $b \in\left(S \cap f \stackrel{\circ}{\sigma}^{q}\right)-X$. Поскольку $m-r-1 \geqslant 2$ и $(n-r-1)+1<m-r-1$, то по соображениям общего положения сушествует дуга $l \subset S$, соединяющая $a$ и $b$, такая, что $l \cap X=\varnothing, l \cap f \sigma^{p}=a$ и $l \cap f \sigma^{q}=b$. Пусть $\beta=R_{S}(l) * D^{r}$. Тогда $\beta \cap f \sigma^{p}$ и $\beta \cap f \sigma^{q}$ являются PL $p$ - и $q$-шарами (рис. 10.1).

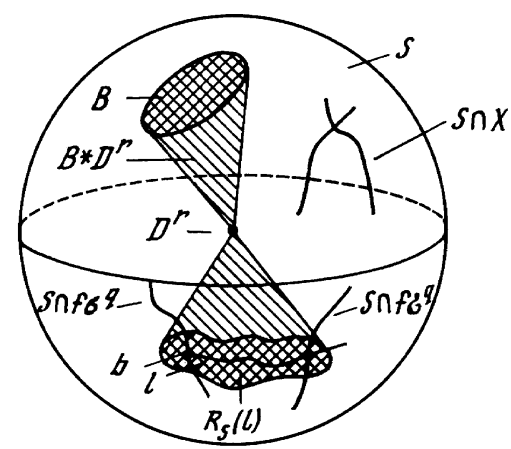

Рис. 10.1 
Построим шар $D^{p}$. По предложению индукции, $f \sigma^{p} \cap f \partial \sigma^{q}=f \partial \sigma^{p} \cap f \sigma^{q}=D^{r}$. Значит, $\Sigma \subset\left(f^{\circ} \stackrel{\circ}{p}^{p} \cap \stackrel{\circ}{\sigma}^{q}\right) \cup D^{r}$. И $f \sigma^{p}$, и $\left(S * D^{r}\right) \cap f \sigma^{p}=\left(S \cap f \sigma^{p}\right) * D^{r}$ являются регулярными окрестностями $D^{r} \mathrm{rel} \partial D^{r}$ в $f \sigma^{p}$. Тогда по лемме о сдавливании 10.4 $f \sigma^{p} \searrow\left(S \cap f \sigma^{p}\right) * D^{r}$ rel $D^{r}$. И $S \cap f \sigma^{p}$, и $R_{S \cap f \sigma} p(a)$ являются регулярными окрестностями $a$ в $S \cap f \sigma^{p}$. Значит, по лемме о сдавливании $10.4 S \cap f \sigma^{p} \searrow R_{S \cap f \sigma^{p}}(a)$. Поэтому

$$
\left(S \cap f \sigma^{p}\right) * D^{r} \searrow R_{S \cap f \sigma^{p}}(a) * D^{r}=\beta \cap f \sigma^{p} \operatorname{rel} D^{r} .
$$

Пусть $C_{1}-$ след $\Sigma$ при этой последовательности сдавливаний

$$
f \sigma^{p} \searrow\left(S \cap f \sigma^{p}\right) * D^{r} \searrow \beta \cap f \sigma^{p} \operatorname{rel} D^{r}
$$

находяшийся в обшем положении (рис. 10.2). Пусть $D^{p}=R_{f \sigma^{p}}\left(\left(\beta \cap f \sigma^{p}\right) \cup C_{1}, D^{r}\right)$ (регулярные окрестности, определяющие шар $D^{p}$ и шары $D^{q}, D^{m}$, ниже должны браться в сужениях одной и той же триангуляции пространства $\mathbb{R}^{m}$ ). Тогда для $D^{p}$ верны свойства 1) и 4) второй леммы о шаре 10.3 , а также
1) $C_{1} \subset f \sigma^{p}$;
2) $\Sigma \subset\left(\beta \cap f \sigma^{p}\right) \cup C_{1}$;
3) $D^{p}-$ PL $p$-шар;
4) $C_{1} \cap X=\varnothing$;
5) $D^{p} \cap X \subset D^{r}$;
6) $C_{1} \cap f \sigma^{q}=\Sigma$.

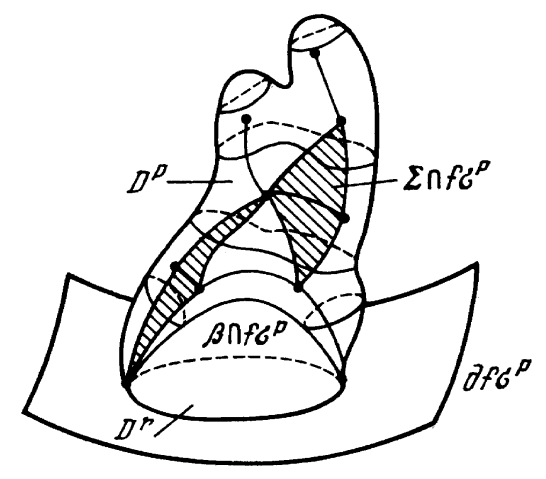

Рис. 10.2

Действительно, свойства (10.2.1) и (10.2.2) очевидны. Поскольку $\Sigma \subset D^{r} \cup f^{\circ} \sigma^{p}$, то $C_{1} \subset D^{r} \cup f \stackrel{\circ}{\sigma^{p}}$, значит, свойство 1) второй леммы о шаре верно. Поскольку $f \sigma^{p}$ есть $\mathrm{PL}$-многообразие и $f \sigma^{p} \searrow\left(\beta \cap f \sigma^{p}\right) \cup C_{1}$ rel $D^{r}$, то $f \sigma^{p}$ - регулярная окрестность $\left(\beta \cap f \sigma^{p}\right) \cup C_{1}$ в $f \sigma^{p} \operatorname{rel} D^{r}$ [20; теорема 9.1]. Тогда по [20; теорема 3.1] сушествует изотопия $G_{t}: f \sigma^{p} \rightarrow f \sigma^{p} \operatorname{rel}\left(\beta \cap f \sigma^{p}\right) \cup C_{1}$ между $G_{0}=$ id и гомеоморфизмом $G_{1}: f \sigma^{p} \cong D^{r} \operatorname{rel}\left(\beta \cap f \sigma^{p}\right) \cup C_{1}$. Отсюда следует свойство (10.2.3). Более того, $\left.G_{1}\right|_{\partial f \sigma^{p}}$ - гомеоморфизм $\partial f \sigma^{p} \cong \partial D^{p} \operatorname{rel} D^{r}$. Поскольку $D^{r}$ незаузлен в $\partial f \sigma^{p}$, то свойство 4) второй леммы о шаре верно для $D^{p}$. По соображениям общего положения $\operatorname{dim} \Sigma \leqslant 2 n-m$. Тогда $\operatorname{dim} C_{1} \leqslant 2 n-m+1$. По соображениям общего положения 
и поскольку $n+(2 n-m)<m$, имеем $\Sigma \cap X=\varnothing$. Снова соображения общего положения и $n+(2 n-m+1)<m$ влекут свойство (10.2.4). Так как $l \cap X=\varnothing$, то $\beta \cap f \sigma^{p} \cap X=D^{r}$. Это и свойства (А), (10.2.4) влекут свойство (10.2.5). По определению относительного сдавливания $C_{1} \cap D^{r}=\Sigma \cap D^{r}$. Отсюда и по соображениям общего положения $(n+(2 n-m+2)<m)$ мы имеем свойство (10.2.6).

Аналогично строятся такие полиэдры $C_{2}$ и $D^{q}$, что свойства 1) и 4) второй леммы о шаре и (10.2.1)-(10.2.6) верны для $C_{1} \rightarrow C_{2}$ и $p \rightarrow q$.

Построим теперь шар $D^{m}$. Возьмем PL $(m-r-1)$-шар $B \subset S-\left(l \cup f \sigma^{p} \cup f \sigma^{q}\right)$. Тогда $S-\stackrel{\circ}{B}$ является PL $(m-r-1)$-шаром и $\sigma^{m}=\left(\mathbb{R}^{m} \cup \infty\right)-\operatorname{Int}\left(B * D^{r}\right)$ является PL $m$-шаром. По свойствам (10.2.1) и (А) $C_{1} \cap\left(S * D^{r}\right) \subset\left(S \cap f \sigma^{p}\right) * D^{r}$. Поэтому $C_{1} \cap \operatorname{Int}\left(B * D^{r}\right)=\varnothing$ и, значит, $C_{1} \subset \stackrel{\circ}{\sigma}^{m} \cup D^{r}$. Аналогично, $C_{2} \subset \stackrel{\circ}{\sigma} m \cup D^{r}$. Аналогично построению $D^{p}, D^{q}$ пусть $C$ - след полиэдра $C_{1} \cup C_{2}$ при последовательности сдавливаний

$$
\sigma^{m} \searrow \sigma^{m} \cap\left(S * D^{r}\right)=(S-\stackrel{\circ}{B}) * D^{r} \searrow R_{S}(l) * D^{r}=\beta \operatorname{rel} D^{r},
$$

находящийся в общем положении. Аналогично свойствам (10.2.1)-(10.2.3) доказьвается, что $C \subset \sigma^{m} \cup D^{r}, C_{1} \cup C_{2} \subset \beta \cup C$ и $D^{m}=R_{\sigma^{m}}\left(\beta \cup C, D^{r}\right)$ является PL $m$-шаром. Аналогично свойству (10.2.4), используя это свойство и $n+(2 n-m+2)<m$, доказывается, что $C \cap X=\varnothing$. Тогда свойство 6) второй лемма о шаре доказывается аналогично свойству (10.2.5). Свойство (10.2.6) и соображения обшего положения влекут

$$
C \cap f \sigma^{q}=\left(C_{1} \cup C_{2}\right) \cap f \sigma^{q}=C_{2} \cup\left(C_{1} \cap f \sigma^{q}\right)=C_{2} \cup \Sigma=C_{2} .
$$

Аналогично, $C \cap f \sigma=C_{1}$. Тогда $(\beta \cup C) \cap f \sigma^{p}=\left(\beta \cap f \sigma^{p}\right) \cup C_{1}$ и $(\beta \cup C) \cap f \sigma^{q}=$ $\left(\beta \cap f \sigma^{q}\right) \cup C_{2}$. Поскольку $D^{p}, D^{q}$ и $D^{m}$ регулярные окрестности rel $D^{r}$ полиэдров $\left(\beta \cap f \sigma^{p}\right) \cup C_{1},\left(\beta \cap f \sigma^{q}\right) \cup C_{2}$ и $\beta \cup C$ в сужениях одной и той же триангулящии пространства $\mathbb{R}^{m}$ на $f \sigma^{p}, f \sigma^{q}$ и $\sigma^{m}$ соответственно, отсюда следует свойство 2) второй леммы о шаре. По свойству (10.2.2) и определению $D^{p}, D^{q}, \Sigma$

$$
\left(\partial D^{p}-D^{r}\right) \cap\left(\partial D^{q}-D^{r}\right) \subset\left(f \stackrel{\circ}{\sigma}^{p}-\Sigma\right) \cap\left(f \stackrel{\circ}{\sigma}^{q}-\Sigma\right)=\varnothing .
$$

Значит, свойство 3) второй леммы о шаре верно. По свойству (10.2.1) мы имеем $\Sigma \subset\left(\beta \cap f \sigma^{p}\right) \cup C_{1} \subset \beta \cup C \subset \stackrel{\circ}{D}^{m} \cup D^{r}$, т.е. свойство 5$)$ второй леммы о шаре верно.

\section{§ 11. Идея доказательства теоремы 4.2.e}

Незамкнутьй случай теоремы 4.2.е следует из теорем 4.2.і и 11.1.а. Доказательство замкнутого случая теоремы 4.2.е состоит из трех частей: построения погружения $h: N-\stackrel{\circ}{B}^{n} \rightarrow \mathbb{R}^{m}$ (теорема 4.2.i), построения квазивложения $g: N \rightarrow \mathbb{R}^{m}$ (здесь мы доказываем более слабую теорему 11.1.b, полное доказательство см. в [137]) и построения вложения $f: N \rightarrow \mathbb{R}^{m}$ (лемма 11.2, при применении леммы 11.2 нужно заметить, что для получения свойства $f \simeq{ }_{\mathrm{eq}} \Phi$ в теореме 4.2.е нужно взять триангуляцию $T$ многообразия $N$ такую, что $B$ содержится в единственном симплексе триангулящии $T$, тогда $\widetilde{f} \simeq_{\text {eq }} \widetilde{g} \simeq_{\text {eq }} \Phi$ на $\widetilde{T}$ и $\widetilde{N}$ эквивариантно ретрагируется на $\left.\widetilde{T}[63]\right)$. Незамкнутый случай теоремы 4.7.е следует из теорем 4.7.і и 11.1.b. Замкнутый случай теоремы 4.7.e 
доказьвается аналогично теореме 4.2.е с использованием теоремы "Конкордантность Влечет Изотопию" (§ 1), и мы не приводим доказательство (см. [137]).

Теорема 11.1. Пусть $N$ есть $n$-полиэдр (DIFF $n$-многообразие).

а) [46; теоремы 2'а и 6.4], [153; теорема 8], [55; следствие 5.(ii)] Предположим, что либо $m \geqslant \frac{3(n+1)}{2}$, либо $\left[N-P L(D I F F) n\right.$-многообразие, $(N, \partial N)\left[\frac{d}{3}\right]$-связно, $\pi_{1} \partial N=0$ и $\left.m \geqslant 6\right]$. Ecли $h: N \rightarrow \mathbb{R}^{m}$ есть $P L(D I F F)$ погружение $и \Phi: \widetilde{N} \rightarrow S^{m-1}$ - такое әквивариантное отображение, что $\widetilde{h} \simeq_{\mathrm{eq}} \Phi$ на $S N$, mо $h$ будет $P L$ $(D I F F)$ регулярно гомотопно $P L(D I F F)$ вложению $f: N \rightarrow \mathbb{R}^{m}$ такому, что $\widetilde{f} \simeq$ eq $\Phi$.

b) Предположим, что $m \geqslant n+3$ и $N$ - замкнутое $d$-связное $P L$ многообразие. Eсли $h: N \rightarrow \mathbb{R}^{m}$ есть PL погружение $и \Phi: \widetilde{N} \rightarrow S^{m-1}$ - такое әквивариантное отображение, что $\widetilde{h} \simeq_{\mathrm{eq}} \Phi$ на $S N$, то $h$ PL регулярно гомотопно $P L$ отображению $f: N \rightarrow \mathbb{R}^{m}$ такому, что $\Sigma(g)$ содержится в некотором $P L$-иаре $B \subset N$ u $\widetilde{f} \simeq_{\mathrm{eq}} \Phi$ на $\widetilde{N}-\widetilde{B}$.

DIFF случай теоремы 4.1.е был получен из DIFF случаев теорем 4.1.i и 11.1.a [46]. PL случай теоремы 4.1.е аналогичным образом доказан в [137] (первоначально он был доказан похожим, но немного другим образом [153], [136]). Для $A \subset N$ обозначим $A^{*}=A \times N \cup N \times A$. Следуюший результат доказан в [58]. Для полноты мы приводим его доказательство.

ЛЕмма 11.2 (ср. [135; теорема 2.1.2]). Предположим, ито $N$ - замкнутое гомологически $d$-связное $P L$ п-многообразие, $m-n \geqslant 3 u g: N \rightarrow \mathbb{R}^{m}$ - такое отображение, что $\Sigma(g)$ содержится в некотором $P L n$-иаре $B \subset N$. Тогда существует вложсние $f: N \rightarrow \mathbb{R}^{m}$ такое, что $f=g$ на $N-\stackrel{\circ}{B}$.

ДОКАЗАТЕЛЬСТвО ТЕОРЕм 4.2.і и 4.7.i ДЛЯ PL МНОГООБРАЗИЙ. Мы следуем идее [52]. Эквивариантное многообразие Штифеля $V_{m n}^{\mathrm{eq}}$ - пространство эквивариантных (относительно антиподальных инволюций) отображений $S^{n-1} \rightarrow S^{m-1}$. $P L$ многообразие Iтияфеля $V_{m n}^{P L}$ - пространство эквивариантных PL вложений $S^{n-1} \rightarrow S^{m-1}$ (очевидно, что $V_{m n}^{P L}$ есть ретракт пространства всех PL вложений $\left.S^{n-1} \rightarrow S^{m-1}\right)$.

Множество эквивариантных отображений $\Phi: S N \rightarrow S^{m-1}$ с точностью до эквивариантной гомотопии находится во взаимно однозначном соответствии с множеством сечений $V_{m n}^{\mathrm{eq}}$-расслоения над $N$, ассоциированного с $T N$, с точностью до послойной гомотопии. По [54] $\mathrm{PL}$ погружения $N \rightarrow \mathbb{R}^{m}$ с точностью до PL регулярной гомотопии взаимно однозначно соответствуют сечениям $V_{m n}^{P L}$-подрасслоения вышеописанного расслоения. Препятствия к деформированию сечения $V_{m n}^{\mathrm{eq}}$-расслоения в сечение $V_{m n}^{P L}$-подрасслоения лежат в

$$
H^{i}\left(N, \pi_{i}\left(V_{m n}^{\mathrm{eq}}, V_{m n}^{P L}\right)_{T}\right) \cong H_{n-i}\left(N, \partial N, \pi_{i}\left(V_{m n}^{\mathrm{eq}}, V_{m n}^{P L}\right)\right)
$$

(коэффициенты скручены в соответствии с ориентирующим двойным накрытием многообразия $N ; \partial N=\varnothing$ возможно). По формуле универсальных коэффициентов достаточно доказать, что $\pi_{i}\left(V_{m n}^{\mathrm{eq}}, V_{m n}^{P L}\right)=0$ для $0 \leqslant i \leqslant 2(m-n)-2$. Из точной 
последовательности тройки $V_{m n}^{\mathrm{eq}} \supset V_{m n}^{P L} \supset V_{m n}$ получаем, что достаточно доказать, что $\pi_{i}\left(V_{m n}^{\mathrm{eq}}, V_{m n}\right)=0$ для $0 \leqslant i \leqslant 2(m-n)-2$ и $\pi_{i}\left(V_{m n}^{P L}, V_{m n}\right)=0$ для $0 \leqslant i \leqslant 2(m-n)-3$. Первое следует из [52; $(1.1)]$ (доказательство с исправленными опечатками приведено также в [137]) и гомотопической точной последовательности пары $V_{m n}^{\mathrm{eq}} \supset V_{m n}$ (даже для $\left.0 \leqslant i \leqslant 2(m-n)-1\right)$. По [50; 8.15, 10.2, 11.2], [47] $\pi_{i}\left(V_{m n}^{P L}, V_{m n}\right)=C_{i-1}^{m-n}=0$ для $i \leqslant 2(m-n)-3, i \leqslant n$ (даже при $\left.m-n=2\right)$.

Докажем последнее без использования неопубликованного результата из [50]. По [47; 4.6], [106; теорема 5.1] существуют гомоморфизмы $\pi_{i}\left(V_{m n}\right) \stackrel{\alpha_{i}}{\rightarrow} \pi_{i}\left(G_{m}, G_{m-n}\right) \stackrel{\beta_{i}}{\longleftarrow}$ $\pi_{i}\left(V_{m n}^{P L}\right)$ такие, что $\alpha_{i}$ - изоморфизм для $0 \leqslant i \leqslant 2(m-n)-4$ и эпиморфизм для $i=$ $2(m-n)-3$ и $\beta_{i}-$ изоморфизм для $0 \leqslant i \leqslant 2 m-n-4$ и эпиморфизм для $i=2 m-n-3$. Легко проверить, что гомоморфизм включения $\rho_{i}^{P L}: \pi_{i}\left(V_{m n}\right) \rightarrow \pi_{i}\left(V_{m n}^{P L}\right)$ совпадает с $\beta_{i}^{-1} \circ \alpha_{i}$, поэтому $\rho_{i}^{P L}$ - изоморфизм для $0 \leqslant i \leqslant 2(m-n)-4$ и эпиморфизм для $i=2(m-n)-3$. Остается применить точную гомотопическую последовательность пары $V_{m n}^{\mathrm{eq}} \supset V_{m n}^{P L}$.

Более прямое и короткое доказательство теоремы 4.2.i, без использования результатов о кусочно-линейных многообразиях Штифеля, приведено в [137]. Это доказательство теоремы 4.2.і в некотором смысле аналогично доказательствам DIFF случая теоремы 4.1.i [139], [56], [52], [113] и PL случая теоремы 4.1.і [55].

ДОКАЗАТЕЛЬСТВо ТЕОРЕМЫ 11.1.b. Если фиксирована триангуляция $T$ полиэдра $N$, обозначим $T N=\bigcup_{\sigma \cap \tau \neq \varnothing} \sigma \times \tau$. Зафиксируем такую триангуляцию $T$ многообразия $N$, что $h$ невырожденно (т.е. сужение $h$ на любой симплекс триангулящии $T$ является вложением), $\widetilde{\Delta}(h) \cap T N=\varnothing_{\text {и }} \widetilde{h} \simeq_{\text {еq }} \Phi$ на $T N \cap \widetilde{T}$. Применяя первую часть доказательства теоремы 4.1.e, можно регулярно гомотопировать $h$ в $T$-погружение $g: N \rightarrow \mathbb{R}^{m}$, для которого $g \sigma \cap g \tau=\varnothing$ для любых непересекающихся симплексов $\sigma, \tau \in T$ таких, что $\operatorname{dim} \sigma \leqslant \operatorname{dim} \tau$ и $\operatorname{dim} \sigma+2 \operatorname{dim} \tau \leqslant 2 m-3$. Пусть $K=T^{(2 m-2 n-3)}$. Так как $n+(2 m-2 n-3) \leqslant 2 m-n-3$, то $\widetilde{\Delta}(g) \cap K^{*}=\varnothing$. Так как $g-$ невырожденное погружение, то $\widetilde{\Delta}(g) \cap R\left(K^{*}\right)=\varnothing$ и $\widetilde{g} \simeq_{\mathrm{eq}} \Phi$ на $R\left(K^{*}\right) \cap \widetilde{T}$. (Если $h-$ погружение, то $\widetilde{\Delta}(h)$ замкнуто в $N \times N$ и, вообще, $\mathrm{Cl}_{N \times N} \widetilde{\Delta}(f) \subset \widetilde{\Delta}(f) \cup \operatorname{diag} N$. Если $E \supset T N$, то $R_{\widetilde{T}}(E) \cap \widetilde{N}$ эквивариантно ретрагируется на $E \cap \widetilde{T}$ для любого подполиэдра $E \subset_{\text {eq }} N \times N$.) Заметим, что $R\left(K^{*}\right) \cap \widetilde{T} \supset(R(K))^{*} \cap \widetilde{T}$. Следовательно, $\Sigma(g) \cap R(K)=\varnothing$. Действительно, $N-R(K)$ - регулярная окрестность в $N$ двойственного к $K$ остова $U$. Так как $\operatorname{dim} U=n-1-(2 m-2 n-3)=d$ и $N$ $d$-связно, из леммы о поглощении 8.1 получаем, что $U$ содержится в некотором PL $n$-шаре в $N$. Следовательно, по теореме о единственности регулярной окрестности $N-R(K)$ также содержится в некотором (возможно, другом) PL $n$-шаре $B \subset N$. Мы имеем $\Sigma(g) \subset N-R(K) \subset B$ и $\widetilde{g} \simeq_{\text {eq }} \Phi$ на $(R(K))^{*} \cap \widetilde{N} \supset \widetilde{N}-(N-R(K)) \sim \supset \widetilde{N}-\widetilde{B}$.

ДокАЗАТЕЛЬСТво ЛЕммы 11.2. Пусть $M=\mathbb{R}^{m}-\operatorname{Int} R(g(N-\stackrel{\circ}{B}), g \partial B)$. Так как $N$ гомологически $d$-связно, по двойственности Александера получаем

$$
H_{i}(M) \cong H^{m-1-i}\left(\mathbb{R}^{m}-M\right) \cong H^{m-1-i}(N-\stackrel{\circ}{B}) \cong H_{n-m+1+i}(N)=0
$$

для $i \leqslant 2 n-m+1$. Так как $m-n \geqslant 3$, то $M$ односвязно. Следовательно, из гомоморфизма Гуревича получаем, что $M(2 n-m+1)$-связно. Значит, по теореме Ирвина о вложении 3.2.b вложение $g: \partial B \rightarrow \partial M$ продолжается до вложения $f: B \rightarrow M$. Продолжая $f$ как $g$ вне $B$, мы завершаем доказательство. 


\section{§12. Идея доказательства изотопических версий}

Изотопические версии теорем о вложимости сводятся к их граничным версиям (но не к относительныл версиям, как написано в [153]) с помощью теоремы "Конкордантность Влечет Изотопность" (§1). Для иллюстрации идеи приведем

ДОКАЗАТЕЛЬСТво PL СЛУЧАЯ ТЕОРЕмЫ 3.6.1. Рассмотрим вложения $f, g: N^{n}$ $\rightarrow \mathbb{R}^{m}$. Возьмем гомотопию $H: N \times I \rightarrow \mathbb{R}^{m} \times I$ общего положения между $f$ и $g$. Используя доказательство теоремы 3.2, модифицируем отображение $H$ во вложение. Поскольку $f$ и $g$ - вложения, то $H$ - вложение в некоторой окрестности оснований $N \times 0 \cup N \times 1$. Поэтому мы можем совершать модификации отображения $H$, необходимые для доказательства теоремы 3.2 , в $\operatorname{Int}(N \times I)$, чтобы получить вложение $H^{\prime}$, совпадаюшее с $H$ на $N \times\{0,1\}$. Это вложение $H^{\prime}: N \times I \rightarrow \mathbb{R}^{m} \times I$-конкордантность между $f=\left.H^{\prime}\right|_{N \times 0}$ и $g=\left.H^{\prime}\right|_{N \times 1}$. Значит, $f$ и $g$ изотопны по теореме "Конкордантность Влечет Изотопность".

Для подполиэдра $A \subset K$ обозначим $A^{*}=\widetilde{A} \cup[A \times(K-A)] \cup[(K-A) \times A]$.

ДоКАЗАТЕЛЬСТВо РL СЛУЧАЯ ТЕОРЕМЫ 4.6.е. Возьмем гомотопию $H: N \times I \rightarrow$ $\mathbb{R}^{m} \times I$ общего положения между $f$ и $g$. Применяя лемму о цилиндре 12.2 (см. ниже), получим эквивариантное отображение $\Sigma \Phi \circ p: \widehat{N \times I} \rightarrow S^{m}$ такое, что $\Sigma \Phi \circ p \simeq_{\text {eq }} \widetilde{H}$ на $(N \times\{0,1\})^{*}$. Применяя теорему 12.1 (PL случай граничной версии теоремы 4.1.е) к $K=N \times I$ и $A=N \times\{0,1\}$, получаем конкордантность между $f$ и $g$. Значит, $f$ и $g$ изотопны по теореме "Конкордантность Влечет Изотопность".

TЕорема 12.1. Пусть $K$ есть n-полиэдр и $m \geqslant \frac{3(n+1)}{2}, A-$ подполиэдр полиәдра $K, B^{m}-P L$-иар, $g: K \rightarrow B^{m}-P L$ отображские такое, что $\left.g\right|_{A}$ вложение в $\partial B^{m}$ u $g(K-A) \subset \stackrel{\circ}{B^{m}}$. Вложсние $f: K \rightarrow B^{m}$ maкое, что $\left.f\right|_{A}=\left.g\right|_{A}$ и $f(K-A) \subset \stackrel{\circ}{B^{m}}$, существует тогда и только тогда, когда әквивариантное

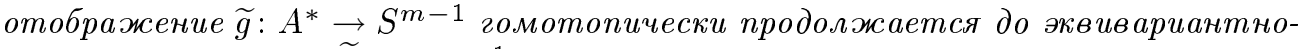
го отображения $\Phi: \widetilde{K} \rightarrow S^{m-1}$.

Теорема 12.1 доказьвается аналогично теореме 4.1.е.

В этом параграфе для полиэдра $N$ с фиксированной триангулящией $T$ мы отождествляем $\widetilde{T}$ и $\widetilde{N}$ (это не приведет к неудобствам).

ЛЕмма О цилиндРЕ 12.2 ([136; лемма 6.1]; ср. [153; лемма 7.1], [135; лемма 3.3]). Если полиэдр $N$ имеет фиксированную триангуляцию и $N \times I$ имеет клеточное подразделение произведения, то

$$
\frac{\widetilde{N \times I}}{N \times N \times 0 \times 1, N \times N \times 1 \times 0} \cong_{\mathrm{eq}} \Sigma(\tilde{N} \times I) .
$$

Обозначим через $p: \widetilde{N \times I} \rightarrow \Sigma(\widetilde{N} \times I)$ фактор-проекиию. Тогда

$$
p^{-1} \Sigma(\tilde{N} \times\{0,1\})=\widehat{N \times I} \cap(N \times N \times \partial(I \times I)) .
$$

Далее, предположим, чтто $H: N \times I \rightarrow \mathbb{R}^{m} \times I \subset \mathbb{R}^{m+1}$ - сохраняющее уровни $P L$ отобрахсение $и \varphi: \widetilde{N} \times \operatorname{diag} I \rightarrow S^{m-1}$ - эквивариантная гомотопия между $\widetilde{\left.H\right|_{N \times 0}}$ и $\widehat{\left.H\right|_{N \times 1}}$. Тогда $\widetilde{H} \simeq$ eq $\Sigma \varphi \circ p н а(N \times\{0,1\})^{*}$. 


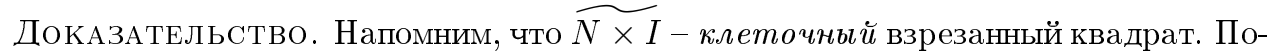
этому для любых $(x, s, y, t) \in \widehat{N \times I}$ либо $x \neq y$, либо $\{s, t\}=\{0,1\}$. Определим отображение $p: \widetilde{N \times I} \rightarrow \Sigma(\tilde{N} \times I)$ формулой

$$
p(x, s, y, t)= \begin{cases}{\left[\left(x, y, \frac{s+t}{2}\right), s-t\right],} & x \neq y, \\ {[\tilde{N} \times I, s-t],} & x=y(\text { и, значит, }|s-t|=1)\end{cases}
$$

(рис. 12.1). Легко видеть, что $p$ корректно определено, эквивариантно, сюръективно и что нетривиальные $p$-прообразы имеют только вершины надстройки и эти $p$-прообразы суть $N \times N \times 0 \times 1$ и $N \times N \times 1 \times 0$. Утверждение о $p^{-1} \Sigma$ легко проверяется.

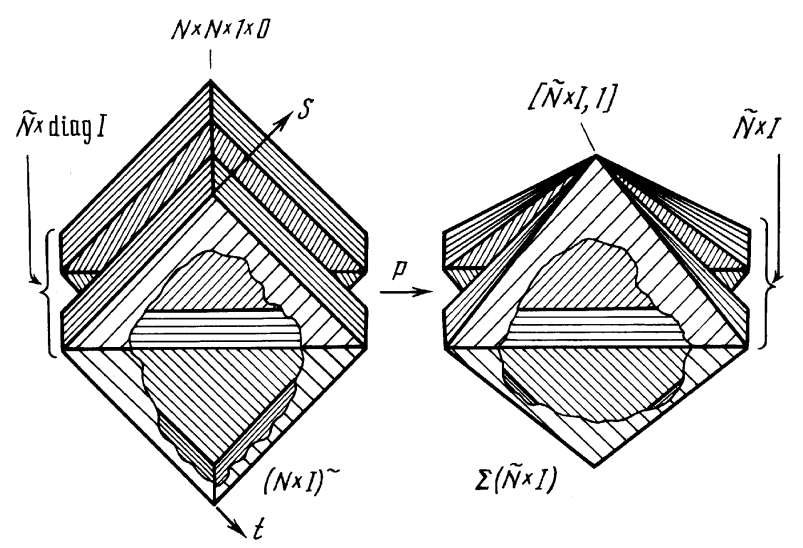

Рис. 12.1

Для доказательства части "далее", заметим, что $\Sigma \varphi \circ p(x, t, y, t)=\varphi(x, y, t)$. Значит, $\Sigma \varphi \circ p=\widetilde{H}$ на $(N \times\{0,1\})^{*} \cap(\widetilde{N} \times \operatorname{diag} I)$. Для $(x, s, y, t) \in(N \times\{0,1\})^{*}$ и $s<t$ $(s>t)$ обе точки $\Sigma \varphi \circ p(x, s, y, t)$ и $\widetilde{H}(x, s, y, t)$ лежат в северной (южной) открытой полусфере и не являются антиподальными. Поэтому $\Sigma \varphi \circ p \simeq$ eq $\widetilde{H}$ на $(N \times\{0,1\})^{*}$.

Мы благодарим В. А. Васильева, С.А.Мелихова, Е. Рафикова и Ю. П. Соловьева за полезные обсуждения и замечания, а также С. А. Мелихова за предоставление записей лекций второго автора по $\S 8$ и Е. Рафикова за перевод части текста на русский язык.

\section{СПИСОК ЛИТЕРАТУРЫ}

[1] Adachi M. Embeddings and Immersions. Providence, RI: Amer. Math. Soc., 1993. (Transl. of Math. Monographs. V. 124.)

[2] Akhmetiev P., Repovš D., Skopenkov A. Embedding products of low-dimensional manifolds in $\mathbb{R}^{m} / /$ Topology Appl. (to appear).

[3] Akhmetiev P., Repovš D., Skopenkov A. Obstructions to approximating maps of surfaces in $\mathbb{R}^{4}$ by embeddings // Topology Appl. (submitted).

[4] Akin E. Manifold phenomena in the theory of polyhedra // Trans. Amer. Math. Soc. 1969. V. 143. P. 413-473.

[5] Арнольд В.И.Проблема 6 // Матем. просвещение. 1958. № 3. С. 273. 
[6] Арнольд В.И.О представлении непрерьвных функций трех переменных суперпозициями непрерывных функций двух переменных // Матем. сб. 1959. Т. 48. № 1. С. 3-74; 1962. T. 56. № 3. C. 392 .

[7] Boechat J. Plongements différentiables de variétés de dimension $4 k$ dans $\mathbb{R}^{6 k+1} / /$ Comment Math. Helv. 1971. V. 46. P. 141-161.

[8] Boechat J., Haefliger A. Plongements differentiables de varietes orientees de dimension 4 dans $\mathbb{R}^{7}$ // Essays on Topology and Related Topics, Mem. dedié à G. de Rham. New York: Springer-Verlag, 1970. P. 156-166.

[9] Borsuk K. Über stetige Abbildungen der euklidischen Räume // Fund. Math. 1933. V. 21. P. $236-246$.

[10] Brodsky N., Repovš D., Skopenkov A. B. A classification of 3-thickenings of 2-polyhedra // Topology Appl. 1999. V. 94. P. 307-314.

[11] Brown R. L. Immersions and embeddings up to cobordism // Canad. J. Math. 1971. V. 23. P. 1102-1115.

[12] Bryant J. L. Approximating embeddings of polyhedra in codimension 3 // Trans. Amer. Math. Soc. 1972. V. 170. P. 85-95.

[13] Cappell S. E., Shaneson J. L. Imbeddings and immersions of 4-dimensional manifolds in $\mathbb{R}^{6} / /$ Geometric Topology / ed. J.C. Cantrell. New York: Academic Press, 1979. P. 301-303.

[14] Casson A. J. Three lectures on new infinite constructions in 4-dimensional manifolds // A la Récherche de la Topologie Perdue, Progr. Math. / ed. L. Guillou, A. Marin. V. 62. Boston: Birkhäuser, 1986. P. 201-244 (рус. перевод в сборнике: В поисках утраченной топологии, ред. Л. Гийу, А. Марен, М.: Мир, 1989).

[15] Cavicchioli A., Repovš D., Skopenkov A.B. Open problems on graphs, arising from geometric topology // Topology Appl. 1998. V. 84. P. 207-226.

[16] Чернавский А. В. Топологические вложения полиэдров в евклидовы пространства // Докл. АН СССР. 1965. Т. 6. С. 1606-1610.

[17] Чернавский А. В. Кусочно-линейные аппроксимации вложений клеток и сфер в коразмерности больше двух // Матем. сб. 1969. Т. 80. С. 339-364.

[18] Claytor S. Topological immersions of peanian continua in a spherical surface // Ann. of Math. (2). 1934. V. 35. P. 809-835.

[19] Claytor S. Peanian continua not embeddable in a spherical surface // Ann. of Math. (2). 1937. V. 38. P. 631-646.

[20] Cohen M. M. A general theory of relative regular neighbourhoods // Trans. Amer. Math. Soc. 1969. V. 136. P. 189-230.

[21] Cohen R. L. The immersion conjecture for differentiable manifolds // Ann. of Math. (2). 1985. V. 122. P. 237-328.

[22] Conner P. E., Floyd E. E. Fixed points free involutions and equivariant maps // Bull. Amer. Math. Soc. 1960. V. 66. P. 416-441.

[23] Daverman R. J. Decompositions of Manifolds. Orlando, Fla.: Academic Press, 1986.

[24] Donaldson S. K. The orientation of Yang-Mills moduli spaces and 4-manifold topology // J. Differential Geom. 1987. V. 26. P. 397-428.

[25] Dranišnikov A. N., Repovš D., Ščepin E. V. On intersection of compacta in Euclidean space: the metastable case // Tsukuba J. Math. 1993. V. 17. P. 549-564.

[26] Edwards R. D. The equivalence of close piecewise linear embeddings // Gen. Topology Appl. 1975. V. 5. P. 147-180.

[27] Fang F. Embedding four manifolds in $\mathbb{R}^{7} / /$ Topology. 1994. V. 33. P. 447-454.

[28] Flores A. Über $n$-dimensionale Komplexe die im $E^{2 n+1}$ absolute Selbstverschlungen sind // Ergeb. Math. Koll. 1934. V. 6. P. 4-7.

[29] Freedman M. H., Krushkal V.S., Teichner P. Van Kampen's embedding obstruction is incomplete for 2-complexes in $\mathbb{R}^{4} / /$ Math. Res. Lett. 1994. V. 1. P. 167-176.

[30] Freedman M. H., Quinn F. Topology of 4-Manifolds. Princeton: Princeton Univ. Press, 1990 . 
[31] Фукс Д. Б., Шварц А. С.Циклические степени полиэдров и проблема вложения // Докл. АН СССР. 1959. Т. 125. С. 285-288.

[32] Galecki M. On embeddability of CW-complexes in Euclidean space // Preprint. Knoxville: Univ. of Tennessee, 1992.

[33] Gillman D., Matveev S. V., Rolfsen D. Collapsing and reconstruction of manifolds // Contemp. Math. 1994. V. 164. P. 35-39.

[34] Gillman D., Rolfsen D. Three-manifolds embed in small 3-complexes // Internat. J. Math. 1992. V. 3. P. 179-183.

[35] Gitler S. Immersion and embedding of manifolds // Proc. Sympos. Pure Math. 1971. V. 22. P. 87-96.

[36] Gluck H. Unknotting $S^{1}$ in $S^{4} / /$ Bull. Amer. Math. Soc. 1963. V. 69. P. 91-94.

[37] Gluck H. Geometric characterisation of differentiable manifolds in Euclidean space, II // Michigan Math. J. 1968. V. 15. P. 33-50.

[38] Gordon C. M. A. Embeddings piecewise linear manifolds with boundary // Proc. Cambridge Philos. Soc. 1972. V. 72. P. 21-25.

[39] Громов М. Дифференциальные соотношения с частными производными. М.: Мир, 1990.

[40] Habegger N., Kaiser U. Link homotopy in the 2-metastable range // Topology. 1998. V. 37. P. 75-94.

[41] Hacon D. D. J. Embeddings of $S^{p}$ in $S^{1} \times S^{q}$ in the metastable range // Topology. 1968 . V. 7. P. $1-10$.

[42] Haefliger A. Plongements différentiables de variétés dans variétés // Comment. Math. Helv. 1961. V. 36. P. 47-82.

[43] Haefliger A. Knotted $(4 k-1)$-spheres in $6 k$-space // Ann. of Math. (2). 1962. V. 75. P. 452-466.

[44] Haefliger A. Plongements de variétés dans le domaine stable // Sem. Bourbaki 1964. V. 15 (1962/63). № 245.

[45] Haefliger A. Differentiable links // Topology. 1962. V. 1. P. 241-244.

[46] Haefliger A. Plongements différentiables dans le domaine stable // Comment. Math. Helv. 1962. V. 37. P. 155-176.

[47] Haefliger A. Differentiable embeddings of $S^{n}$ in $S^{n+q}$ for $q>2 / /$ Ann. of Math. (2). 1966. V. 83. P. 402-436.

[48] Haefliger A. Differentiable embeddings of $S^{n} / /$ Bull. Amer. Math. Soc. 1961. V. 67. P. 109-112.

[49] Haefliger A. Lissage des immersions, I // Topology. 1967. V. 6. P. 221-239.

[50] Haefliger A. Lissage des immersions, II // Preprint, 1966.

[51] Haefliger A. Knotted spheres and related geometric problems // Proc. Internat. Congr. Math., Moscow, 1966, 1968. P. 437-445.

[52] Haefliger A., Hirsch M. W. Immersions in the stable range // Ann. of Math. (2). 1962. V. 75. P. 231-241.

[53] Haefliger A., Hirsch M. W. On the existence and classification of differentiable embeddings // Topology. 1963. V. 2. P. 129-135.

[54] Haefliger A., Poénaru V. La classification des immersions combinatoires // Inst. Hautes Etudes Sci. Publ. Math. 1964. V. 23. P. 75-91.

[55] Harris L.S. Intersections and embeddings of polyhedra // Topology. 1969. V. 8. P. 1-26.

[56] Hirsch M.W. Immersions of manifolds // Trans. Amer. Math. Soc. 1959. V. 93. P. 242-276.

[57] Hirsch M. W. The embedding of bounding manifolds in Euclidean space // Ann. of Math. 1961. V. 74. P. 494-497.

[58] Hirsch M.W. On embedding 4-manifolds in $\mathbb{R}^{7} / /$ Proc. Cambridge Philos. Soc. 1965. V. 61. P. $657-658$

[59] Hirsch M.W., Zeeman E. C. Engulfing // Bull. Amer. Math. Soc. 1966. V. 72. P. 113-115.

[60] Horvatič K. On embedding polyhedra and manifolds // Trans. Amer. Math. Soc. 1971. V. 157. P. 417-436. 
[61] Hsiang W. C., Levine J., Szczarba R.H. On the normal bundle of a homotopy sphere embedded in Euclidean space // Topology. 1965. V. 3. P. 173-181.

[62] Ху С. Ц. Теория гомотопий. М.: Мир, 1964.

[63] Hu S. T. Isotopy invariants of topological spaces // Proc. Roy. Soc. London Ser. A. 1960. V. 255. P. 331-366.

[64] Hudson J. F.P. Knotted tori // Topology. 1963. V. 2. P. 11-22.

[65] Hudson J. F. P. Non-embedding theorem // Topology. 1963. V. 2. P. 123-128.

[66] Hudson J. F. P. Extending piecewise linear isotopies // Proc. London Math. Soc. (3). 1966. V. 16. P. $651-668$.

[67] Hudson J. F. P. PL embeddings // Ann. of Math. (2). 1967. V. 85. №1. P. 1-31.

[68] Hudson J.F.P. On sunny collapsing // Proc. Cambridge Philos. Soc. 1969. V. 66. P. $247-250$.

[69] Hudson J. F. P. Piecewise Linear Topology. New York: Benjamin, 1969.

[70] Hudson J. F. P. Concordance, isotopy and diffeotopy // Ann. of Math. (2). 1970. V. 91. P. $425-448$.

[71] Hudson J. F. P. Obstructions to embedding disks // Topology of Manifolds, Proc. Univ. Georgia, 1969, 1971. P. 407-415.

[72] Hudson J. F. P. Embeddings of bounded manifolds // Proc. Cambridge Philos. Soc. 1972. V. 72. P. $11-20$.

[73] Hudson J.F.P., Lickorish W.B.R. Extending piecewise linear concordances // Quart. J. Math. (2). 1971. V. 22. P. 1-12.

[74] Hudson J. F. P., Zeeman E. C. On combinatorial isotopy // Inst. Hautes Etudes Sci. Publ. Math. 1964. V. 19. P. 69-94.

[75] Hudson J.F.P., Zeeman E. C. On regular neighborhoods // Proc. London Math. Soc. (3). 1964. V. 14. P. 719-745; Correction // ibid. 1970. V. 21. P. 513-524.

[76] Гуревич В., Волмэн Г. Теория размерности. М.: ИЛ, 1948.

[77] Husch L.S. $\varepsilon$-maps and embeddings // Res. Exp. Math. 1988. V. 16. P. 273-280.

[78] Irwin M.C. Embeddings of polyhedral manifolds // Ann. of Math. (2). 1965. V. 82. P. $1-14$.

[79] James I. M. The Topology of Stiefel Manifolds. London: London Math. Soc., 1976.

[80] van Kampen E. R. Komplexe in euklidischen Räumen // Abh. Math. Sem. Univ. Hamburg. 1932. V. 9. P. 72-78; 152-153.

[81] Kearton C. Obstructions to embeddings and isotopy in the metastable range // Math. Ann. 1979. V. 243. P. 103-113.

[82] Kervaire M. A. An interpretation of G. Whitehead's generalization of H. Hopf's invariant // Ann. of Math. (2). 1959. V. 69. P. 345-365.

[83] Kervaire M. A. Smooth homology spheres and their fundamental groups // Trans. Amer. Math. Soc. 1969. V. 144. P. 67-72.

[84] Kervaire M. A., Milnor J. W. On 2-spheres in 4-manifolds // Proc. Nat. Acad. Sci. U.S.A. 1961. V. 47. P. 1651-1657.

[85] Kirby R. 4-manifold problems // Contemp. Math. 1984. V. 35. P. 513-528.

[86] Kirby R. C. The Topology of 4-Manifolds. Berlin: Springer-Verlag, 1989. (Lecture Notes in Math., V. 1374.)

[87] Колмогоров А.Н. О представлении непрерывных функций нескольких переменных в виде суперпозиций непрерывных функций одного переменного и сложения // Докл. АН CCCP. 1957. T. 114. № 5. C. 953-956.

[88] Koschorke U. Link homotopy with many components // Topology. 1991. V. 30. № 2. P. 267-281.

[89] Kuratowski K. Sur le problème des courbes gauches en topologie // Fund. Math. 1930. V. 15. P. 271-283.

[90] Kurlin V. Basic embeddings into products of graphs // Topology Appl. 1999 (to appear).

[91] Курлин В., Скопенков А. Базисные вложения графов в плоскость // 9-я летняя конференция Турнира Городов: Изд-во МцНМО, 1998. С. 34-44, 106-113.

[92] Lackenby M. The Whitney trick // Topology Appl. 1996. V. 71. P. 115-118. 
[93] Lannes J. La conjecture des immersions // Astérisque. 1982. V. 92/93. P. 331-346.

[94] Levin M. Dimension and superposition of continuous functions // Israel J. Math. 1990. V. 70. № 2. P. 205-218.

[95] Levine J. Unknotting spheres in codimension 2 // Topology. 1965. V. 4. P. 9-16.

[96] Lickorish W. B. R. The piecewise linear unknotting of cones // Topology. 1965. V. 4. P. 67-91.

[97] Lickorish W. B. R., Siebenmann L. C. Regular neighborhoods and the stable range // Trans. Amer. Math. Soc. 1969. V. 139. P. 207-230.

[98] Mardešić S., Segal J. A note on polyhedra embeddable in the plane // Duke Math. J. 1966. V. 33. P. 633-638.

[99] Mardešić S., Segal J. $\varepsilon$-mappings and generalized manifolds // Michigan Math. J. 1967. V. 14. P. 171-182.

[100] Massey W.S. On the Stiefel-Whitney classes of a manifold, 1 // Amer. J. Math. 1960. V. 82. P. 92-102.

[101] Massey W.S. On the Stiefel-Whitney classes of a manifold, 2 // Proc. Amer. Math. Soc. 1962. V. 13. P. 938-942.

[102] Massey W. Homotopy classification of 3-component links of codimension greater than 2 // Topology Appl. 1990. V. 34. P. 269-300.

[103] McLane S., Adkisson V.W. Extensions of homeomorphisms on the spheres // Lectures on Topology. Ann. Arbor MI: Univ. of Michigan Press, 1941. P. 223-230.

[104] Miller R. T. Close isotopies on piecewise-linear manifolds // Trans. Amer. Math. Soc. 1970. V. 151. P. 597-628.

[105] Miller R. T. Approximating codimension 3 embeddings // Ann. of Math. (2). 1972. V. 95. P. 406-416.

[106] Millett K. C. Piecewise linear embeddings of manifolds // Illinois J. Math. 1975. V. 19. P. 354-369.

[107] Милнор Дж., Сташефф Дж. Характеристические классы. М.: Мир, 1979.

[108] Neuwirth L. An algorithm for the construction of 3-manifolds from 2-complexes // Proc. Cambridge Philos. Soc. 1968. V. 64. P. 603-613.

[109] Новиков С.П. Вложения односвязных многообразий в евклидовы пространства // Докл. АН СССР. 1961. Т. 138. С. 775-778.

[110] Ostrand P. A. Dimension of metric spaces and Hilbert's problem 13 // Bull. Amer. Math. Soc. 1965. V. 7. P. 619-622.

[111] Paechter G. F. On the groups $\pi_{r}\left(V_{m n}\right)$, I-V // Quart. J. Math. Oxford Ser. (2). 1956. V. 7. P. $249-265 ;$ 1958. V. 9. P. 8-27; 1959. V. 10. P. $17-37$; 1959. V. 10. P. 241-260; 1960. V. 11. P. 1-16.

[112] Penrose R., Whitehead J.H.C., Zeeman E. C. Embeddings of manifolds in a Euclidean space // Ann. of Math. (2). 1961. V. 73. P. 613-623.

[113] Poénaru V. Regular homotopy and isotopy // Mimeographed notes: Harvard Univ., 1964.

[114] Понтрягин Л.С. Характеристические циклы гладких многообразий // Докл. АН CCCP. 1942. T. 35. № 2. C. 35-39.

[115] Постников М. М. Теория гомотопий клеточных пространств. М.: Наука, 1985.

[116] Rees E. Some embeddings of Lie groups in Euclidean spaces // Mathematika (London). 1971. V. 18. P. 152-156.

[117] Rees E. Problems concerning embeddings of manifolds // Adv. in Math. (China). 1990. V. 19. P. $72-79$.

[118] Repovš D., Skopenkov A. B. Embeddability and isotopy of polyhedra in Euclidean spaces // Proc. Steklov Inst. Math. 1996. V. 212. P. 173-188.

[119] Repovš D., Skopenkov A. B. A deleted product criterion for approximability of a map by embeddings // Topology Appl. 1998. V. 87. P. 1-19.

[120] Repovš D., Skopenkov A. B. Borromean rings and embedding obstructions // Proc. Math. Inst. Russ. Acad. Sci. (to appear). 
[121] Repovธ̌ D., Skopenkov A. B. Projected embeddings and desuspension of $\alpha$-invariant // Preprint.

[122] Repovš D., Skopenkov A. B., Ščepin E. V. On embeddability of $X \times I$ into Euclidean space // Houston J. Math. 1995. V. 21. P. 199-204.

[123] Рохлин В.А. Вложение неориентируемых трехмерных многообразий в пятимерное евклидово пространство // Докл. АН СССР. 1965. Т. 160. С. 549-551.

[124] Rourke C.P., Sanderson B. J. Block bundles, I // Ann. of Math. (2). 1968. V. 87. P. 1-28.

[125] Рурк К. П., Сандерсон Б. Дж. Введение в кусочно-линейную топологию. М.: Мир, 1974.

[126] Rushing T. B. Topological Embeddings. New York: Academic Press, 1973.

[127] Sarkaria K. S. A one-dimensional Whitney trick and Kuratowski's graph planarity criterion // Israel J. Math. 1991. V. 73. P. 79-89.

[128] Sarkaria K.S. Kuratowski complexes // Topology. 1991. V. 30. P. 67-76.

[129] Шепин Е. В., Штанько М. А. Спектральньй критерий вложимости компактов в евклидовы пространства // Труды Ленингр. междун. топол. конф. Л.: Наука, 1983. C. $135-142$.

[130] Segal J., Skopenkov A., Spież S. Embeddings of polyhedra in $\mathbb{R}^{m}$ and the deleted product obstruction // Topology Appl. 1998. V. 85. P. 335-344.

[131] Segal J., Spież S. Quasi embeddings and embeddings of polyhedra in $\mathbb{R}^{m} / /$ Topology Appl. 1992. V. 45. P. 275-282.

[132] Shapiro A. Obstructions to the embedding of a complex in a Euclidean space, I: The first obstruction // Ann. of Math. (2). 1957. V. 66. P. 256-269.

[133] Скопенков А. Геометрическое доказательство теоремы Нойвирта об утолщаемости 2-мерных полиэдров // Матем. заметки. 1994. Т. 56. № 2. С. 94-98.

[134] Skopenkov A. B. A description of continua basically embeddable in $\mathbb{R}^{2} / /$ Topology Appl. 1995. V. 65. P. 29-48.

[135] Skopenkov A. B. On the deleted product criterion for embeddability of manifolds in $\mathbb{R}^{m}$ // Comment. Math. Helv. 1997. V. 72. P. 543-555.

[136] Skopenkov A.B. On the deleted product criterion for embeddability in $\mathbb{R}^{m} / /$ Proc. Amer. Math. Soc. 1998. V. 126. P. 2467-2476.

[137] Skopenkov A. On the deleted product criterion for embeddings and immersions of manifolds in $\mathbb{R}^{m} / /$ Topology. (submitted).

[138] Skopenkov A. On the generalized Massey-Rolfsen invariant for link maps in $\mathbb{R}^{m} / /$ Fund. Math. (submitted).

[139] Smale S. The classification of immersions of spheres in Euclidean spaces // Ann. of Math. (2). 1959. V. 69. P. 327-344.

[140] Spież S., Toruńczyk H. Moving compacta in $\mathbb{R}^{m}$ apart // Topology Appl. 1991. V. 41. P. 193-204.

[141] Stallings J. Polyhedral homotopy-spheres // Bull. Amer. Math. Soc. 1960. V. 66. P. $485-488$.

[142] Stallings J. On topologically unknotted spheres // Ann. of Math. (2). 1963. V. 77. P. $490-503$.

[143] Stallings J. Homology and central series of groups // J. Algebra. 1965. V. 2. P. 170-181.

[144] Sternfeld Y. Dimension, superposition of functions and separation of points in compact metric spaces // Israel J. Math. 1985. V. 50. P. 13-53.

[145] Sternfeld Y. Hilbert's 13th problem and dimension // Lecture Notes in Math. 1989. V. 1376. P. 1-49.

[146] Szücs A. The Gromov-Eliashberg proof of Haefliger's theorem // Studia Sci. Math. Hungar. 1982. V. 17. P. 303-318.

[147] Taniyama K. Homology classification of spatial embeddings of a graph // Topology Appl. 1995. V. 65. P. 205-228.

[148] Тода Х. Композиционные методы в теории гомотопических групп сфер. М.: Наука, 1982.

[149] Васильев В. А. Топология дополнений к дискриминантам. М.: Фазис, 1997. 
[150] Wall C. T. C. All 3-manifolds imbed in 5-space // Bull. Amer. Math. Soc. 1965. V. 71. P. $490-503$.

[151] Wall C. T. C. Classification problems in differential topology. IV: Thickenings // Topology. 1966. V. 5. P. 73-94.

[152] Wall C. T. C. Surgery on Compact Manifolds. London: Academic Press, 1970.

[153] Weber C. Plongements de polyèdres dans le domaine metastable // Comment. Math. Helv. 1967. V. 42. P. 1-27.

[154] Whitehead J. H. C. Simplicial spaces, nuclei and $m$-groups // Proc. London Math. Soc. 1939. V. 45. P. 243-327.

[155] Whitehead J.H.C. On the groups $\pi_{r}\left(V_{m}, V_{n}\right)$ and sphere bundles // Proc. London Math. Soc. (2). 1944. V. 48. P. 243-291; correction // ibid. 1945. V. 49. P. 479-481.

[156] Whitney H. Differentiable manifolds in Euclidean space // Proc. Nat. Acad. Sci. U.S.A. 1935. V. 21. № 7. P. 462-464.

[157] Whitney H. On the topology of differentiable manifolds // Lectures on Topology. Ann Arbor, MI: Univ. of Michigan Press, 1941. P. 101-141.

[158] Whitney H. The self-intersections of a smooth $n$-manifolds in $2 n$-space // Ann. of Math. (2). 1944. V. 45. P. 220-246.

[159] Wu W.T. On the realization of complexes in Euclidean spaces, I-III // Sci. Sinica. 1958. V. 7. P. 251-297; P. 365-387; 1959. V. 8. P. 133-150.

[160] Wu W. T. A Theory of Embedding, Immersion and Isotopy of Polytopes in an Euclidean Space. Peking: Science Press, 1965.

[161] Yang C. T. On theorems of Borsuk-Ulam, Kakutani-Yamabe-Yujobo and Dyson, I // Ann. of Math. (2). 1954. V. 60. P. 262-282.

[162] Zeeman E. C. Unknotting spheres // Ann. of Math. (2). 1960. V. 72. P. 350-360.

[163] Zeeman E. C. Isotopies and knots in manifolds // Topology of 3-Manifolds and Related Topics / ed. M. K. Fort. Englewood Cliffs: Prentice-Hall, 1962.

[164] Zeeman E. C. The Poincare conjecture for $n \geqslant 5 / /$ Topology of 3-Manifolds and Related Topics / ed. M. K. Fort. Englewood Cliffs: Prentice-Hall, 1962.

[165] Zeeman E.C. Unknotting combinatorial balls // Ann. of Math. (2). 1963. V. 78. P. 501-526.

[166] Zeeman E. C. Seminar on Combinatorial Topology. Paris: Inst. Hautes Etudes Sci., 1963 (Ch. 1-6), 1965 (Ch. 7), 1966 (Ch. 8).

[167] Zhongmou L. Every 3-manifold with boundary embeds in Triod $\times$ Triod $\times I / /$ Proc. Amer. Math. Soc. 1994. V. 122. P. 575-579.

Institute for Mathematics, Physics and Mechanics,

Поступила в редакцию University of Ljubljana, Slovenia;

12.08.1999

Kolmogorov College, Moscow

E-mail: dusan.repovs@fmf.uni-lj.si;

skopenko@aesc.msu.ru, skopenko@mccme.ru 